\title{
Heat kernel expansion: user's manual
}

\author{
D.V. Vassilevich ${ }^{\mathrm{a}, \mathrm{b}}$ \\ ${ }^{a}$ Institut für Theoretische Physik, Universität Leipzig, Augustusplatz 10, D-04109 \\ Leipzig, Germany \\ ${ }^{\mathrm{b}}$ V.A. Fock Insitute of Physics, St. Petersburg University, 198904 St. Petersburg, \\ Russia
}

\begin{abstract}
The heat kernel expansion is a very convenient tool for studying one-loop divergences, anomalies and various asymptotics of the effective action. The aim of this report is to collect useful information on the heat kernel coefficients scattered in mathematical and physical literature. We present explicit expressions for these coefficients on manifolds with and without boundaries, subject to local and non-local boundary conditions, in the presence of various types of singularities (e.g., domain walls). In each case the heat kernel coefficients are given in terms of several geometric invariants. These invariants are derived for scalar and spinor theories with various interactions, Yang-Mills fields, gravity, and open bosonic strings. We discuss the relations between the heat kernel coefficients and quantum anomalies, corresponding anomalous actions, and covariant perturbation expansions of the effective action (both "low-" and "high-energy" ones).
\end{abstract}

Key words: heat kernel, functional determinants, effective action, boundary conditions, anomalies

PACS: 04.62.+v, 11.10.-z, 02.40.-k

Email address: vassil@itp.uni-leipzig.de (D.V. Vassilevich).

Preprint submitted to Elsevier Science 1 February 2008 


\section{Contents}

\begin{tabular}{lll}
\hline & Introduction & 4
\end{tabular}

2 Spectral functions: heat kernel. zeta function, resolvent 11

2.1 Differential geometrv and the operators of Laplace tvpe . . . . . . . 11

2.2 Spectral functions . . . . . . . . . . . . . . . . . . 14

2.3 Lorentzian signature $\ldots \ldots \ldots \ldots$. . . . . . . . . . . . . . . 17

$\begin{array}{lll}3 & \text { Relevant operators and boundary conditions } & 18\end{array}$

3.1 Scalar fields . . . . . . . . . . . . . . . . . . 18

3.2 Bosonic string . . . . . . . . . . . . . . . . . . . . . . . . . . . . 19

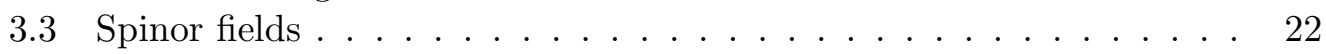

3.4 Vector fields . . . . . . . . . . . . . . . . . . . . . . . . . . . . . . . . . . . . . . . . 25

3.5 Graviton . . . . . . . . . . . . . . . . . . . . . . . . . 28

$4 \quad$ Heat kernel expansion on manifolds without boundarv 31

4.1 General formulad . . . . . . . . . . . . . . . . . 31

4.2 Examples . . . . . . . . . . . . . . . . . . . . . 38

4.2.1 Yang-Mills theorv in flat space . . . . . . . . . . . . . . . 38

$4.2 .2 \quad$ Free fields in curved space . . . . . . . . . . . . . . . . 39

4.3 DeWitt iterative procedure . . . . . . . . . . . . . . . . . . 40

4.4 Non-minimal operators . . . . . . . . . . . . . . . . . . . . . . 41

$5 \quad$ Heat kernel expansion on manifolds with boundaries 43

5.1 Two particular cases . . . . . . . . . . . . . . . . 43

5.2 Dirichlet and Neumann boundary conditions . . . . . . . . . . . . 45

5.3 Mixed boundary conditions . . . . . . . . . . . . . . . 47

5.4 Other boundarv conditions . . . . . . . . . . . . . . . 49

5.4.1 Boundarv conditions with tangential derivatives and Born-Infeld action from open strings

5.4.2 Spectral or Ativah-Patodi-Singer (APS) boundarv conditions 51

6 Manifolds with singularities $\quad 54$

6.1 Non-integrable potentials . . . . . . . . . . . . . . 54

6.2 Conical singularities . . . . . . . . . . . . . . . . . . . . . 55

6.3 Domain walls and brane world . . . . . . . . . . . . . 56

6.4 Non-smooth boundaries . . . . . . . . . . . . . . . . . . . . 60

6.5 Dielectric bodies . . . . . . . . . . . . . . . . 60

7 Anomalies $\quad 62$

7.1 Conformal anomaly . . . . . . . . . . . . . . . . . . . . 62

7.2 Chiral anomalv . . . . . . . . . . . . . . . . . . 65

7.3 Remarks on the Index Theorem . . . . . . . . . . . . . . . . . . 68

\begin{tabular}{|lll}
8 & Resummation of the heat kernel expansion & $\mathbf{7 2}$
\end{tabular}

8.1 Modified large mass expansion . . . . . . . . . . . . . . . . . . . . . . 72

8.2 Covariant perturbation theorv . . . . . . . . . . . . . . 73

8.3 "Low energy" expansion . . . . . . . . . . . . . . . . . . . . . 75

8.4 Heat kernel on homogeneous spaces . . . . . . . . . . . . . . . 77 
$9 \quad$ Exact results for the effective action $\quad 81$ 9.1 The Polvakov action . . . . . . . . . . . . . . . . 81

9.2 Duality svmmetrv of the effective action . . . . . . . . . . . . . . 82

\begin{tabular}{lr}
\hline 10 Conclusions & 88
\end{tabular}

$\begin{array}{lr}\text { References } & 91\end{array}$ 


\section{Introduction}

It was noted by Fock [207] in 1937 that it is convenient to represent Green functions as integrals over an auxiliary coordinate (the so-called "proper time") of a kernel satisfying the heat equation. Later on Schwinger [379] recognised that this representations makes many issues related to renormalization and gauge invariance in external fields more transparent. These two works introduced the heat kernel to quantum theory. DeWitt made the heat kernel one of the main tools of his manifestly covariant approach $[147,148,149,150]$ to quantum field theory and quantum gravity which became dominant for many years.

Heat kernel is a classical subject in mathematics $[271]^{1}$. Asymptotics of the heat kernel are closely related to the eigenvalue asymptotics found by H. Weyl $[418,419]$ and studied further in [325,326]. The problem, as it was formulated by Kac [286], reads: "Can one hear the shape of a drum?". In other words, this is the problem of recovering geometry of a manifold from the spectrum of a natural differential operator. Heat kernel coefficients proved very useful in this context. On the other hand, the heat kernel is also an adequate tool to study the index theorem of Atiyah and Singer $[22,236,18]$.

By about 1990 the heat kernel expansion on manifolds without boundaries or with boundaries and simplest local boundary conditions on them was well understood. Also, the heat kernel became a standard tool in calculations of the vacuum polarisation, the Casimir effect, and in study of quantum anomalies. Later on, progress in theoretical physics, especially in string theory and related areas, and parallel developments in mathematics made this field highly specialised. New results on non-standard boundary conditions (as, e.g., containing tangential derivatives on the boundary or non-localities) or on non-standard geometries (domain walls) were scattered in large amounts of physical and mathematical literature. The aim of this report is to present a unifying approach to the heat kernel expansion and to supply the reader with a "user-friendly" guide to the field. The main idea which we shall pursue is the universality of the heat kernel. A single calculation (though, sometimes, quite involved) may help in a large variety of applications regardless of such details as spin, gauge group, etc. As well, just a single universal object in fact describes counterterms, anomalies, some asymptotics of the effective action, and much more.

To illustrate the use of the heat kernel in quantum field theory let us consider the generating functional for the Green functions of the field $\phi$ in the path integral representation

$$
Z[J]=\int \mathcal{D} \phi \exp (-\mathcal{L}(\phi, J))
$$

$\overline{1}$ A historical survey of the mathematical literature on the heat kernel expansion can be found in [245]. 
The heat kernel methods are almost exclusively used for the one-loop calculations. In this approximation it is enough to expand the action $\mathcal{L}$ to up to the quadratic order in quantum fluctuations $\phi$.

$$
\mathcal{L}=\mathcal{L}_{\mathrm{cl}}+\langle\phi, J\rangle+\langle\phi, D \phi\rangle,
$$

where $\mathcal{L}_{\mathrm{cl}}$ is the action on a classical background, $\langle.,$.$\rangle denotes an inner product$ on the space of quantum fields. Usually, this inner product is just an integral over the underlying space. For real one-component scalar fields it reads

$$
\left\langle\phi_{1}, \phi_{2}\right\rangle=\int d^{n} x \sqrt{g} \phi_{1}(x) \phi_{2}(x) .
$$

The linear term in (1.2) contains in general contributions from the external sources of the field $\phi$ and from the first variation of the classical action. If the background satisfies classical equation of motion for the field $\phi$, the latter part of the linear term vanishes, though the former one (external sources) should be kept arbitrary if one wishes to study correlation functions of $\phi$. We stress that the background and quantum fields may be of completely different nature. For example, it is a meaningful problem to consider pure quantum scalar fields on the background of pure classical gravity. $D$ is a differential operator. After a suitable number of integrations by part it is always possible to convert the quadratic part of the action to the form given in (1.2). We postpone discussion of possible boundary terms to the next sections. In the simplest case of quantum scalar field on the background of a classical geometry $D$ is a Laplacian with a mass term:

$$
D=D_{0}:=-\nabla_{\mu} \nabla^{\mu}+m^{2} .
$$

Just this simple example is enough to illustrate the material of this section. Note, that in this case $J$ has no contribution from the first variation of the classical action since gravity is not quantised.

The path integral measure is defined by:

$$
1=\int \mathcal{D} \phi \exp (-\langle\phi, \phi\rangle) .
$$

Strictly speaking the right hand side of (1.5) is divergent. The essence of the condition (1.5) is that this divergence does not depend on external sources and on the background geometry and, therefore, may be absorbed in an irrelevant normalisation constant. The Gaussian integral (1.1) can be evaluated giving

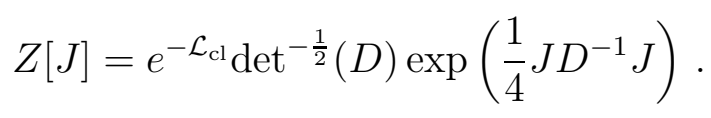

We stress that the equation (1.6) is true only if the operator $D$ is selfadjoint. This means that $D$ is symmetric or formally self-adjoint with respect 
to the scalar product $\langle.,$.$\rangle :$

$$
\left\langle\phi_{1}, D \phi_{2}\right\rangle=\left\langle D \phi_{1}, \phi_{2}\right\rangle
$$

for any $\phi_{1}, \phi_{2}$, and that the domains of definition of $D$ and its adjoint coincide. We will not care about the second requirement since it involves mathematical machinery [364] going beyond the scope of the present report. The first requirement (1.7) poses important restrictions on admissible boundary conditions.

To become convinced that (1.7) is really necessary, one can calculate a finite-dimensional Gaussian integral with

$$
D=\left(\begin{array}{ll}
a & b \\
c & d
\end{array}\right)
$$

and $a, b, c$ and $d$ being real constants first by completing the squares in the exponent, and then compare the result with det $D$ (of course, one should remember a factor of $\pi$ ). The two results coincide if $b=c$.

Let us return to the generating functional (1.6). To analyse the two multiplies on the right hand side of (1.6) which depend on the operator $D$ it is convenient to introduce the heat kernel

$$
K(t ; x, y ; D)=\langle x|\exp (-t D)| y\rangle
$$

This somewhat formal expression means that $K(t ; x, y ; D)$ should satisfy the heat conduction equation

$$
\left(\partial_{t}+D_{x}\right) K(t ; x, y ; D)=0
$$

with the initial condition

$$
K(0 ; x, y ; D)=\delta(x, y)
$$

For $D=D_{0}(1.4)$ on a flat manifold $M=\mathbb{R}^{n}$ the heat kernel reads:

$$
K\left(t ; x, y ; D_{0}\right)=(4 \pi t)^{-n / 2} \exp \left(-\frac{(x-y)^{2}}{4 t}-t m^{2}\right) .
$$

The equations (1.10) and (1.11) can be checked straightforwardly. Let us consider a more general operator $D$ which contains also a potential term or a gauge field. Then (1.12) still describes the leading singularity in the heat kernel as $t \rightarrow 0$. The subleading terms have a form of the power-law corrections ${ }^{2}$ :

$\overline{2}$ On a curved space even the leading term must be modified (cf sec. 4.3). On manifolds with boundaries also half-integer powers of $t$ appear in the expansion, and, consequently, $b_{2 j+1} \neq 0$. 


$$
K(t ; x, y ; D)=K\left(t ; x, y ; D_{0}\right)\left(1+t b_{2}(x, y)+t^{2} b_{4}(x, y)+\ldots\right) .
$$

The coefficients $b_{k}(x, y)$ are regular in the limit $y \rightarrow x$. They are called the heat kernel coefficients ${ }^{3}$. At coinciding arguments $b_{k}(x, x)$ are local polynomials of background fields and their derivatives.

The propagator $D^{-1}(x, y)$ can be defined through the heat kernel by the integral representation

$$
D^{-1}(x, y)=\int_{0}^{\infty} d t K(t ; x, y ; D),
$$

which follows from (1.9) if we suppose that the heat kernel vanishes sufficiently fast as $t \rightarrow \infty$. We can formally integrate the expansion (1.13) to obtain

$$
D^{-1}(x, y) \simeq 2(4 \pi)^{-n / 2} \sum_{j=0}\left(\frac{|x-y|}{2 m}\right)^{-\frac{1}{2} n+j+1} K_{-\frac{1}{2} n+j+1}(|x-y| m) b_{2 j}(x, y),
$$

where $b_{0}=1$. By examining the behaviour of the Bessel function $K_{\nu}(z)$ for small argument $z$ [251] we conclude that the singularities in the propagator at coinciding points are described by the first several heat kernel coefficients $b_{k}$.

Let us consider the part of the generating functional (1.6) which contains $\operatorname{det}(D)$. The functional

$$
W=\frac{1}{2} \ln \operatorname{det}(D)
$$

is called the one-loop effective action. It describes the quantum effects due to the background fields in the one-loop approximation of quantum fields theory. To relate $W$ to the heat kernel we shall use the arguments of [69].

For each positive eigenvalue $\lambda$ of the operator $D$ we may write an identity

$$
\ln \lambda=-\int_{0}^{\infty} \frac{d t}{t} e^{-t \lambda} .
$$

This identity is "correct" up to an infinite constant, which does not depend on $\lambda$ and, therefore, may be ignored in what follows ${ }^{4}$. Now we use $\ln \operatorname{det}(D)=$ $\operatorname{Tr} \ln (D)$ and extend (1.17) to the whole operator $D$ to obtain

$$
W=-\frac{1}{2} \int_{0}^{\infty} \frac{d t}{t} K(t, D)
$$

where

$$
K(t, D)=\operatorname{Tr}\left(e^{-t D}\right)=\int d^{n} x \sqrt{g} K(t ; x, x ; D) .
$$

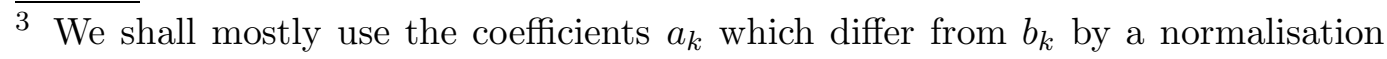
factor.

4 To "prove" this statement one has to differentiate both sides of eq. (1.17) with respect to $\lambda$. 
Here we have only presented some heuristic arguments in favour of eq. (1.18). A more rigorous treatment of functional determinants can be found in sec. 2.2 .

The integral in (1.18) may be divergent at both limits. Divergences at $t=\infty$ are caused by zero or negative eigenvalues of $D$. These are the infra red divergences. They will not be discussed in this section. We simply suppose that the mass $m$ is sufficiently large to make the integral (1.18) convergent at the upper limit. Divergences at the lower limit cannot be removed in such a way. Let us introduce a cut off at $t=\Lambda^{-2}$.

$$
W_{\Lambda}=-\frac{1}{2} \int_{\Lambda^{-2}}^{\infty} \frac{d t}{t} K(t, D) .
$$

It is now easy to calculate the part of $W_{\Lambda}$ which diverges in the limit $\Lambda \rightarrow \infty$ :

$$
\begin{aligned}
W_{\Lambda}^{\mathrm{div}}=-(4 \pi)^{-n / 2} \int d^{n} x \sqrt{g}\left[\sum_{2(j+l)<n} \Lambda^{n-2 j-2 l} b_{2 j}(x, x) \frac{\left(-m^{2}\right)^{l} l !}{n-2 j-2 l}\right. \\
\left.+\sum_{2(j+l)=n} \ln (\Lambda)\left(-m^{2}\right)^{l} l ! b_{2 j}(x, x)+\mathcal{O}\left(\Lambda^{0}\right)\right] .
\end{aligned}
$$

We see that the ultra violet divergences in the one-loop effective action are defined by the heat kernel coefficients $b_{k}(x, x)$ with $k \leq n$.

On non-compact manifolds the integral of $b_{0}(x, x)$ is divergent. This divergence is removed by subtracting a "reference" heat kernel (see sec. 6.1).

Contributions from higher heat kernel coefficients $b_{k}, k>n$ to the effective action are not divergent and can be easily calculated yielding in the limit $\Lambda \rightarrow \infty$

$$
-\frac{1}{2}(4 \pi)^{-n / 2} m^{n} \int d^{n} x \sqrt{g} \sum_{2 j>n} \frac{b_{2 j}(x, x)}{m^{2 j}} \Gamma(2 j-n) .
$$

This is nothing else than the large mass expansion of the effective action. This expansion is valid for relatively weak and slowly varying background fields.

We have seen that the heat kernel expansion describes

- short-distance behaviour of the propagator;

- one-loop divergences and counterterms;

- $1 / m$ expansion of the effective action.

We shall see below that heat kernel provides a natural framework for studying

- quantum anomalies (sec. 7);

- various perturbative expansions of the effective action (sec. 8);

- selected non-perturbative relations for the effective action (sec. 9).

Of course, in all these applications the heat kernel methods have to compete with other techniques. The main advantage of the heat kernel is that it delivers necessary information in terms of just few geometric invariants. This method does not make distinctions between different spins, gauge groups, etc. Even dependence of the space-time dimensionality is in most cases trivial. 
Therefore, on one hand, just a single calculation serves then in many applications. On the other hand, calculations in simple particular cases give valuable information on the general structure of the heat kernel. This property is especially useful when one deals with complicated geometries (like in the presence of boundaries or singularities). During the last decade, many models which lead to such complicated geometries were very actively studied in theoretical physics. The Dirichlet branes and the brane world scenario are the most popular but not the only examples.

We have to mention also the limitations of the heat kernel formalism. It works less effectively in the presence of spinorial background fields, i.e. when there is mixing between bosonic and fermionic quantum fields. This problem is probably of the technical nature, so that the corresponding formalism may be developed some time in the future. A more serious drawback is that the heat kernel expansion is not applicable beyond the one-loop approximation. It is not clear whether necessary generalisations to higher loop could be achieved at all.

It is not possible to write a review paper on heat kernel which would be complete in all respects, especially in the bibliography. A more comprehensive treatment of many mathematical problems related to the heat kernel expansion can be found in [245,297]. The book by Kirsten [297] considers also specific physical applications as the Casimir energy and the Bose-Einstein condensation. The recent review paper [79] is devoted to the Casimir effect (see also [322]). The monographs by Birrell and Davies [69] and Fulling [224] remain standard sources on quantum field theory in curved space. Quantization of gauge theories is explained in [200]. In $[107,198,189,31,191]$ the heat kernel expansion is treated from the point of view of quantum gravity and quantum cosmology. Useful information about properties of the zeta function can be found in [178]. One may also consult [182]. The DeWitt approach to the heat kernel and its' generalisations are described in [55]. The path integral point of view on the heat kernel can be found in [367].

Our primary goal is local formulae for the heat kernel coefficients. Therefore, in some cases global aspects will be somewhat neglected.

This report is organised as follows. The next section contains necessary preliminary information on spectral geometry and differential geometry. There we define main geometric characteristics of the manifold and of the boundary. We discuss the zeta function (which defines the effective action) and the resolvent (which is a generalisation of the propagator). We relate the asymptotics of these functions to the heat kernel coefficients. Through this report we work on Euclidean manifolds. A short remark on the analytical continuation to the Lorentzian signature is given at the end of section 2. In section 3 we consider the most widely used models of quantum field theory and open bosonic strings. The one-loop dynamics in each of these models is defined by a second order differential operator which depends on an effective connection and on a matrix-valued potential. The connection and the potential serve as a basis of an invariant description of all that models in the language of spectral 
geometry. These quantities are written down explicitly for each model. We also define suitable boundary conditions. In section 4 we consider the heat kernel expansion on manifolds without boundary. We introduce a simple and very powerful method and illustrate it by calculating several leading terms in the heat kernel expansion. We also briefly discuss some other methods and non-minimal operators. Section 5 is devoted to manifolds with boundary. The heat kernel expansion for standard Dirichlet, Neumann, and mixed boundary conditions is considered in some detail. We also describe less known oblique and spectral boundary conditions (these are the ones which contain tangential derivatives on the boundary or non-local projectors). We discuss loss of the so-called strong ellipticity for oblique boundary conditions which corresponds to the critical value of the electric field in string physics. With the example of spectral boundary conditions we illustrate appearance of non-standard $(\ln t)$ asymptotics in the heat kernel. The results of sections 4 and 5 are valid on smooth manifolds with smooth potentials and gauge field. In section 6 we consider the case when either the background fields or the manifold itself have singularities. In particular, conical and domain wall singularities are considered.

Sections 7 - 9 are devoted to applications. In section 7 we relate conformal and chiral anomalies to certain heat kernel coefficients and re-derive the anomalies in several particular models. This is a very spectacular but also rather well known application of the heat kernel expansion. In section 8 we go beyond the power series in the proper time $t$. We consider mainly two particular cases. The first one is the case when derivatives are more important than the potentials (so that we sum up leading non-localities). The second one is the so-called low energy expansion which neglects derivatives of the background fields starting with certain order (but treats all powers of background potentials and curvatures exactly). In that section we also review some results on the heat kernel on homogeneous spaces where spectrum of relevant operators may be found exactly. In section 9 we consider two examples when the heat kernel can be used to obtain exact results for the effective action. The first one is the famous Polyakov action. The second example is exact relations between the effective actions in dual theories. In this section (in contrast to section 8) we don't have to neglect derivatives or powers of the background fields. Section 10 contains concluding remarks. 


\section{Spectral functions: heat kernel, zeta function, resolvent}

\subsection{Differential geometry and the operators of Laplace type}

Let $M$ be a smooth compact Riemannian manifold ${ }^{5}$ of dimension $n$ with smooth boundary $\partial M$. We shall also consider the case when the boundary $\partial M$ is empty. Let $V$ be a vector bundle ${ }^{6}$ over $M$. This means that there is a vector space attached to each point of the manifold. For example, this could be a representation space of a gauge group or of the space-time symmetry group. Sections of $V$ are smooth functions bearing a discrete index which could correspond to internal or spin degrees of freedom.

We study differential operators on $V$. We restrict ourselves to second order operators of the Laplace type. Locally such operators can be represented as:

$$
D=-\left(g^{\mu \nu} \partial_{\mu} \partial_{\nu}+a^{\sigma} \partial_{\sigma}+b\right)
$$

where $g^{\mu \nu}$ is the inverse metric tensor on $M ; a^{\sigma}$ and $b$ are matrix valued functions on $M$. There is a unique connection on $V$ and a unique endomorphism $E$ of $V$ (another matrix valued function) so that

$$
D=-\left(g^{\mu \nu} \nabla_{\mu} \nabla_{\nu}+E\right)
$$

where the covariant derivative $\nabla=\nabla^{[R]}+\omega$ contains both Riemann $\nabla^{[R]}$ and "gauge" (bundle) $\omega$ parts. We may express:

$$
\begin{aligned}
& \omega_{\delta}=\frac{1}{2} g_{\nu \delta}\left(a^{\nu}+g^{\mu \sigma} \Gamma_{\mu \sigma}{ }^{\nu} I_{V}\right) \quad \text { and } \\
& E=b-g^{\nu \mu}\left(\partial_{\mu} \omega_{\nu}+\omega_{\nu} \omega_{\mu}-\omega_{\sigma} \Gamma_{\nu \mu}{ }^{\sigma}\right),
\end{aligned}
$$

where

$$
\Gamma_{\nu \mu}{ }^{\sigma}=g^{\sigma \rho} \frac{1}{2}\left(\partial_{\mu} g_{\nu \rho}+\partial_{\nu} g_{\mu \rho}-\partial_{\rho} g_{\mu \nu}\right)
$$

is the Christoffel symbol. $I_{V}$ is the unit operator on $V$.

It is important to understand how general the notion of the Laplace type operator is. The most obvious restriction on $D$ is that it is of second order, i.e. it contains second derivatives, but does not contain higher derivative

5 This simply means that we assume that there is a positive definite metric tensor $g_{\mu \nu}$ on $M$.

6 Many physicists strongly dislike vector bundles. Nevertheless, there are two good reasons for using the fibre bundles in this paper (in parallel with more familiar notations of matrix-valued functions, gauge fields, etc). First, our simplifying comments and examples may help the reader to understand mathematical literature on the subject. Second, one of the main ideas of this report is to reveal some universal structures behind the heat kernel expansion. In particular, we shall see that there is not much difference between different spins and symmetry groups. The vector bundle language seems to be the most adequate language to achieve this goal. The reader may consult the excellent review paper by Eguchi, Gilkey and Hanson [177]. 
parts. In this paper we shall also consider first order operators (Dirac operators, for example). Second, the operator $D$ is a partial differential operator. This excludes negative or fractional powers of the derivatives. The operators containing such structures are called pseudo-differential operators. More information on spectral theory for these operators can be found in [383, 258, 259]. Third, the operator (2.1) has a scalar principal part. This means that the second derivatives in (2.1) are contracted with the metric, and the internal index structure of the second derivative term is trivial. Such operators are also called minimal. Non-minimal operators will be briefly considered in sec 4.4.

We can define local invariants associated with $\omega$ and $g$. Let

$$
R_{\nu \rho \sigma}^{\mu}=\partial_{\sigma} \Gamma_{\nu \rho}^{\mu}-\partial_{\rho} \Gamma_{\nu \sigma}^{\mu}+\Gamma_{\nu \rho}^{\lambda} \Gamma_{\lambda \sigma}^{\mu}-\Gamma_{\nu \sigma}^{\lambda} \Gamma_{\lambda \rho}^{\mu}
$$

be the Riemann curvature tensor, let $R_{\mu \nu}:=R_{\mu \nu \sigma}^{\sigma}$ be the Ricci tensor, and let $R:=R_{\mu}^{\mu}$ be the scalar curvature. With our sign conventions, $R=2$ on the unit sphere $S^{2}$ in the Euclidean space. Let Roman indices $i, j, k$, and $l$ range from 1 through the dimension $n$ of the manifold and index a local orthonormal frame (vielbein) $\left\{e_{1}, \ldots, e_{n}\right\}$ for the tangent space of the manifold. In components we have: $e_{j}^{\mu} e_{k}^{\nu} g_{\mu \nu}=\delta_{j k}, e_{j}^{\mu} e_{k}^{\nu} \delta^{j k}=g^{\mu \nu}$. The inverse vielbein is defined by the relation $e_{\mu}^{j} e_{k}^{\mu}=\delta_{k}^{j}$. These two objects, $e_{\mu}^{k}$ and $e_{j}^{\nu}$, will be used to transform "curved" indices $(\mu, \nu, \rho)$ to "flat" ones $(i, j, k)$ and back. In Euclidean space there is no distinction between upper and lower flat indices.

As usual, the Riemann part of the covariant derivatives contains the Christoffel connection so that

$$
\nabla_{\mu}^{[R]} v_{\nu}=\partial_{\mu} v_{\nu}-\Gamma_{\mu \nu}^{\rho} v_{\rho}
$$

for an arbitrary vector $v_{\nu}$. To extend this derivative to the objects with flat indices one has to use the spin-connection $\sigma_{\mu}$ :

$$
\nabla_{\mu} v^{j}=\partial_{\mu} v^{j}+\sigma_{\mu}^{j k} v_{k}
$$

The condition $\nabla_{\mu} e_{\nu}^{k}=0$ yields

$$
\sigma_{\mu}^{k l}=e_{l}^{\nu} \Gamma_{\mu \nu}^{\rho} e_{\rho}^{k}-e_{l}^{\nu} \partial_{\mu} e_{\nu}^{k} .
$$

Let $\Omega_{\mu \nu}$ be the field strength of the connection $\omega$ :

$$
\Omega_{\mu \nu}=\partial_{\mu} \omega_{\nu}-\partial_{\nu} \omega_{\mu}+\omega_{\mu} \omega_{\nu}-\omega_{\nu} \omega_{\mu} .
$$

The covariant derivative $\nabla$ acts on both space-time and internal indices. For example,

$$
\nabla_{\rho} \Omega_{\mu \nu}=\partial_{\rho} \Omega_{\mu \nu}-\Gamma_{\rho \mu}^{\sigma} \Omega_{\sigma \nu}-\Gamma_{\rho \nu}^{\sigma} \Omega_{\mu \sigma}+\left[\omega_{\rho}, \Omega_{\mu \nu}\right] .
$$

If the boundary $\partial M$ is non-empty we have more invariants. Let $e_{n}$ be inward pointing unit vector field. Let Roman indices $a, b, c$ and $d$ range from 1 to $n-1$ and index a local orthonormal frame for the tangent bundle of $\partial M$. 
Let $L_{a b}:=\Gamma_{a b}^{n}$ be the second fundamental form (extrinsic curvature) of the boundary. We use the Levi-Civita (spin) connections and the connection $\omega$ to covariantly differentiate tensors of all types. Let ';' denote multiple covariant differentiation with respect to the Levi-Civita connection of $M$ and let ':' denote multiple tangential covariant differentiation on the boundary with respect to the Levi-Civita connection of the boundary; the difference between ';' and ':' is measured by the second fundamental form. Thus, for example, $E_{; a}=E_{: a}$ since there are no tangential indices in $E$ to be differentiated. On the other hand, $E_{; a b} \neq E_{: a b}$ since the index $a$ is also being differentiated. More precisely $E_{; a b}=E_{: a b}-L_{a b} E_{; n}$. Since $L$ is only defined on the boundary, this tensor can only be differentiated tangentially.

Consider an example of the circle $S^{1}$ in the plane $\mathbb{R}^{2}$. The line element has the form

$$
(d s)^{2}=(d r)^{2}+r^{2}(d \theta)^{2},
$$

where $0 \leq r<\infty, 0 \leq \theta<2 \pi$. Then $g_{\mu \nu}=\operatorname{diag}\left(1, r^{2}\right)$. $S^{1}$ is defined by the condition $r=r_{0}$. We may choose $e_{\theta}^{1}=r, e_{r}^{2}=-1$ (the minus sign appears because $e^{2}$ is an inward pointing unit vector for the disk with the boundary $S^{1}$ ). Then the second fundamental form of $S^{1}$ is

$$
L_{11}=\left.e_{r}^{2} e_{1}^{\theta} e_{1}^{\theta} \Gamma_{\theta \theta}^{r}\right|_{r=r_{0}}=\frac{1}{r_{0}}
$$

In general, on $S^{n-1}$ considered as a boundary of the ball in $\mathbb{R}^{n}$ the extrinsic curvature is $L_{a b}=\frac{1}{r_{0}} \delta_{a b}$.

If the boundary $\partial M$ is non-empty, one has to define boundary conditions for the field $\phi$. A convenient way to write them down is:

$$
\mathcal{B} \phi=0
$$

where $\mathcal{B}$ is called the boundary operator. In general, the operator $\mathcal{B}$ calculates a linear combination of the boundary data for any given function $\phi$. If $D$ is of Laplace type, the boundary data include value of the function at the boundary and value of it's first normal derivative. The most frequently used choices are the Dirichlet and Neumann boundary operators which we denote $\mathcal{B}^{-}$and $\mathcal{B}^{+}$ respectively:

$$
\begin{aligned}
\mathcal{B}^{-} \phi & =\left.\phi\right|_{\partial M}, \\
\mathcal{B}^{+} \phi & =\left.\left(\phi_{; n}+S \phi\right)\right|_{\partial M},
\end{aligned}
$$

where $S$ is a matrix valued function defined on $\partial M$. The boundary conditions (2.16) are also called Robin or generalised Neumann. In some literature they are called mixed boundary conditions. We shall not use this latter terminology. The name "mixed" is reserved for another type of boundary conditions. Let $\Pi^{-}$and $\Pi^{+}$be two complementary projectors defined on $\left.V\right|_{\partial M},\left(\Pi^{ \pm}\right)^{2}=\Pi^{ \pm}$, $\Pi^{+}+\Pi^{-}=I$. There is a decomposition $\left.V\right|_{\partial M}=V_{N} \oplus V_{D}$, where $V_{N, D}=$ 
$\left.\Pi^{ \pm} V\right|_{\partial M}$. Decompose also $\phi=\phi^{N} \oplus \phi^{D}$ and set

$$
\mathcal{B} \phi:=\left.\phi^{D} \oplus\left(\phi_{; n}^{N}+S \phi^{N}\right)\right|_{\partial M} .
$$

The matrix valued function (endomorphism) $S$ acts on $V_{N}$ only, $S=\Pi^{+} S=$ $S \Pi^{+}$. In other words, we define Dirichlet boundary conditions on $V_{D}$ and generalised Neumann boundary conditions on $V_{N}$. For obvious reason the boundary conditions (2.17) will be called mixed. In sec. 3 we shall see that natural boundary conditions for spinor and vector fields are of this type.

\subsection{Spectral functions}

For the boundary conditions considered in this section as well as on manifolds without a boundary the operator $\exp (-t D)$ with positive $t$ is trace class on the space of square integrable functions $L^{2}(V)$. This means that for an auxiliary smooth function $f$ on $M$

$$
K(t, f, D)=\operatorname{Tr}_{L^{2}}(f \exp (-t D))
$$

is well defined. We also write

$$
K(t, f, D)=\int_{M} d^{n} x \sqrt{g} \operatorname{tr}_{V} K(t ; x, x ; D) f(x),
$$

where $K(t ; x, x ; D)$ is an $y \rightarrow x$ limit of the fundamental solution $K(t ; x, y ; D)$ of the heat equation (1.10) with the initial condition (1.11). If there is a boundary, the kernel $K(t ; x, y ; D)$ should also satisfy some boundary conditions $\mathcal{B}_{x} K(t ; x, y ; D)=0$ in one of the arguments. We stress that $K(t ; x, y ; D)$ is a matrix in the internal indices. $\operatorname{tr}_{V}$ denotes the trace over these indices.

Let $D$ be self-adjoint. This implies that in a suitable basis in the internal space the matrix $\omega_{\mu}$ is anti-hermitian and $E$ is hermitian. Let $\left\{\phi_{\lambda}\right\}$ be a complete basis of orthonormal eigenfunctions of the operator $D$ corresponding to the eigenvalues $\{\lambda\}$. Then

$$
K(t ; x, y ; D)=\sum_{\lambda} \phi_{\lambda}^{\dagger}(x) \phi_{\lambda}(y) e^{-t \lambda} .
$$

We shall almost exclusively work either on manifolds without boundaries, or on manifolds with boundaries with the fields subject to local boundary conditions (2.15), (2.16), or (2.17). In all these cases there is an asymptotic expansion as $t \downarrow 0[381,382,253]^{7}$ :

$$
\operatorname{Tr}_{L^{2}}(f \exp (-t D)) \cong \sum_{k \geq 0} t^{(k-n) / 2} a_{k}(f, D) .
$$

$\overline{7}$ These papers contain also a method allowing to calculate the coefficients of the expansion. The method is, however, too complicated to use it on practice. 
This expansion is valid for almost all boundary conditions appearing in applications to physics. There are, however, some exceptions which will be discussed in sec. 5.4 .

The coefficients $a_{k}$ and the coefficients $b_{k}$ introduced in the previous section (cf. (1.13)) are related by the equation ( $m=0$ for the simplicity):

$$
a_{k}(f, D)=(4 \pi)^{-n / 2} \int_{M} d^{n} x \sqrt{g} b_{k}(x, x) f(x)
$$

Note, that the definition (1.13) is valid on flat manifolds without boundary only (although generalisations to other cases are possible).

The key property of the heat kernel coefficients $a_{k}$ is that they are locally computable in most of the cases. This means that they can be expressed in term of the volume and boundary integrals of local invariants.

For a positive operator $D$ one can define the zeta function $[325,383]$ by the equation:

$$
\zeta(s, f, D)=\operatorname{Tr}_{L^{2}}\left(f D^{-s}\right) .
$$

The zeta function is related to the heat kernel by the integral transformation

$$
\zeta(s, f, D)=\Gamma(s)^{-1} \int_{0}^{\infty} d t t^{s-1} K(t, f, D) .
$$

This relation can be inverted,

$$
K(t, f, D)=\frac{1}{2 \pi i} \oint d s t^{-s} \Gamma(s) \zeta(s, f, D),
$$

where the integration contour encircles all poles of the integrand. Residues at the poles can be related to the heat kernel coefficients:

$$
a_{k}(f, D)=\operatorname{Res}_{s=(n-k) / 2}(\Gamma(s) \zeta(s, f, D)) .
$$

In particular,

$$
a_{n}(f, D)=\zeta(0, f, D) .
$$

Zeta-functions can be used to regularize the effective action [165, 274]. The regularization is achieved by shifting the power of $t$ in (1.18):

$$
W_{s}=-\frac{1}{2} \tilde{\mu}^{2 s} \int_{0}^{\infty} \frac{d t}{t^{1-s}} K(t, D)
$$

where $\tilde{\mu}$ is a constant of the dimension of mass introduced to keep proper dimension of the effective action. The regularization is removed in the limit $s \rightarrow 0$. Eq. (2.28) can be considered as a definition of the regularized effective action without any reference to (1.18). One can also rewrite (2.28) in terms of the zeta function:

$$
W_{s}=-\frac{1}{2} \tilde{\mu}^{2 s} \Gamma(s) \zeta(s, D),
$$

where $\zeta(s, D):=\zeta(s, 1, D)$. 
The gamma function has a simple pole at $s=0$ :

$$
\Gamma(s)=\frac{1}{s}-\gamma_{E}+\mathcal{O}(s),
$$

where $\gamma_{E}$ is the Euler constant. The regularized effective action (2.29) has also a pole at $s=0$ :

$$
W_{s}=-\frac{1}{2}\left(\frac{1}{s}-\gamma_{E}+\ln \tilde{\mu}^{2}\right) \zeta(0, D)-\frac{1}{2} \zeta^{\prime}(0, D) .
$$

According to (2.27) the divergent term in the zeta function regularization is proportional to $a_{n}(D)$ (cf. (1.21) for another regularization scheme). The pole term in (2.31) has to be removed by the renormalization. The remaining part of $W_{s}$ at $s=0$ is the renormalised effective action:

$$
W^{\mathrm{ren}}=-\frac{1}{2} \zeta^{\prime}(0, D)-\frac{1}{2} \ln \left(\mu^{2}\right) \zeta(0, D),
$$

where we have introduced a rescaled parameter $\mu^{2}=e^{-\gamma_{E}} \tilde{\mu}^{2}$. In this approach $\mu^{2}$ describes the renormalization ambiguity which must be fixed by a suitable normalisation condition. Let us remind that here we are working on a compact manifold. On non-compact manifolds $\zeta(s, D)$ may have divergent contributions proportional to the volume. Such divergences are usually removed by the subtraction of a "reference" heat kernel (see sec. 6.1).

Together with (1.16) equation (2.32) yields a definition of the functional determinant for a positive elliptic second order operator which is frequently used in mathematics:

$$
\ln \operatorname{det}(D)=-\zeta^{\prime}(0, D)-\ln \left(\mu^{2}\right) \zeta(0, D) .
$$

Note, that the definitions (2.23), (2.33) are valid for positive operators only. Elliptic 2nd order differential operators have at most finite number of zero and negative modes ${ }^{8}$ which must be treated separately. However, one can extend the definition of the zeta function to operators with negative modes:

$$
\zeta(s, D)=\sum|\lambda|^{-s},
$$

where the sum extends over all non-zero eigenvalues $\lambda$. One can also define another spectral function in a similar way:

$$
\eta(s, D)=\sum \operatorname{sign}(\lambda)|\lambda|^{-s} .
$$

This function is especially useful in spectral theory of Dirac type operators where $\eta(0, \not D)$ measures asymmetry of the spectrum.

8 On manifolds with boundaries one has to require strong ellipticity of the boundary value problem (cf. sec. 5.4). 
Another function which is frequently used especially in the mathematical literature is the resolvent (or, more precisely, its' powers):

$$
\mathrm{R}^{l}(z):=\left(D+z^{2}\right)^{-l} .
$$

If $D$ is on operator of Laplace type subject to "good" boundary conditions 9 and if $l$ sufficiently large $(l>n / 2)$ there is a full asymptotic expansion

$$
\operatorname{Tr}\left(\mathrm{R}^{l}(z)\right)=\sum_{k} \frac{\Gamma\left(l+\frac{k-n}{2}\right)}{\Gamma(l)} a_{k}(D) z^{-2 l+n-k}
$$

as $z \rightarrow \infty$. The coefficients $a_{k}$ are the same as in the heat kernel expansion $(2.21)$.

\subsection{Lorentzian signature}

Locality of the heat kernel coefficients in the Euclidean domain can be easily understood by examining the free heat kernel (1.12). For small $t$ the first term in the exponential strongly suppresses non-local contributions. For Lorentzian metrics the squared distance function $(x-y)^{2}$ is no longer positive definite. Therefore, the simple arguments given above do not work. A partial solution to this problem is to consider the "Schrödinger" equation

$$
\left(i \partial_{\tau}-D\right) \tilde{K}(\tau ; x, y ; D)=0
$$

for the kernel $\tilde{K}$ instead of the heat conduction equation (1.10). Then $\tilde{K}$ oscillates at large distances. However, even though non-local contributions to $\tilde{K}$ oscillate furiously as $\tau \rightarrow 0$ they are not small. Consequently, local asymptotic series do not exist in many cases. A discussion on this point can be found in chapter 9 of [224].

Of course, the heat kernel expansion can be used also on Lorentzian manifolds at least for the renormalization theory where non-local terms are of less importance. Counterterms are still defined by the same heat kernel coefficients with the same functional dependence on the Lorentzian metric. Explicit definitions with the imaginary "proper time" $\tau$ can be found in $[147,69]$. One should note that some background fields receive an imaginary phase when being continued to the Euclidean domain (cf. secs. 3.2 and 3.3).

$\overline{9}$ A more precise meaning of this restriction will be explained in sec. 5.4. 


\section{Relevant operators and boundary conditions}

The operator of Laplace type is not necessarily the scalar Laplacian. In fact, in almost all models of quantum field theory the one loop effective action is defined by an operator of this type. This can be demonstrated by bringing relevant operators to the canonical form (2.2). In this section we give explicit construction of the connection $\omega$ and the endomorphism (matrix-valued potential) $E$ for scalar, spinor, vector and graviton fields. We also describe appropriate boundary conditions.

\subsection{Scalar fields}

Consider first the example of the multi-component real scalar field $\Phi^{A}$ in $n$ dimensions. The action reads

$$
\mathcal{L}=\int_{M} d^{n} x \sqrt{g}\left(g^{\mu \nu} \nabla_{\mu} \Phi^{A} \nabla_{\nu} \Phi^{A}+U(\Phi)+\xi R \Phi^{A} \Phi^{A}\right),
$$

where $\xi$ is the conformal coupling parameter, $U$ is a potential. The covariant derivative $\nabla_{\mu}$ contains the background gauge field $G_{\mu}^{A B}: \nabla_{\mu} \Phi^{A}=\partial_{\mu} \Phi^{A}+$ $G_{\mu}^{A B} \Phi^{B} . G_{\mu}$ is antisymmetric in internal indices $A, B$. To evaluate the oneloop effective action one should expand (3.1) around a background field $\bar{\Phi}$, $\Phi=\bar{\Phi}+\phi$, and keep the terms quadratic in fluctuations:

$$
\begin{aligned}
\mathcal{L}_{2}= & \int_{M} d^{n} x \sqrt{g} \phi^{A}\left(-\left(\nabla^{\mu} \nabla_{\mu}\right)^{A B}+\frac{1}{2}\left(U(\bar{\Phi})^{\prime \prime}\right)^{A B}+\xi R \delta^{A B}\right) \phi^{B} \\
& -\int_{\partial M} d^{n-1} x \sqrt{h} \phi^{A} \nabla_{n} \phi^{A},
\end{aligned}
$$

$h$ is the determinant of the induced metric on the boundary. The inner product for quantum fields $\phi$ reads

$$
<\phi_{1}, \phi_{2}>=\int_{M} d^{n} x \sqrt{g} \phi_{1}^{A} \phi_{2}^{A}
$$

The operator $D$ is defined by the bulk part of the action (3.2):

$$
D^{A B}:=-\left(\nabla^{\mu} \nabla_{\mu}\right)^{A B}+\frac{1}{2}\left(U(\bar{\Phi})^{\prime \prime}\right)^{A B}+\xi R \delta^{A B} .
$$

For a special choice of the parameter $\xi$ :

$$
\xi=\frac{n-2}{4(n-1)}
$$

the operator $D(3.4)$ is conformally covariant (if also $U(\bar{\Phi})^{\prime \prime}=0$ ). To bring the operator (3.4) to the canonical form (2.2) we introduce

$$
\omega_{\mu}^{A B}=G_{\mu}^{A B}, \quad E^{A B}=-\frac{1}{2}\left(U(\bar{\Phi})^{\prime \prime}\right)^{A B}-\xi R \delta^{A B} .
$$


For this case, $\Omega_{\mu \nu}$ is just the ordinary Yang-Mills field strength.

The operator (3.4) is symmetric with respect to the inner product (3.3) if the surface integral

$$
\int_{\partial M} d^{n-1} x \sqrt{h}\left(\phi_{1}^{A} \nabla_{n} \phi_{2}^{A}-\phi_{2}^{A} \nabla_{n} \phi_{1}^{A}\right)
$$

vanishes for arbitrary $\phi_{1}$ and $\phi_{2}$ belonging to its' domain of definition. This may be achieved if one imposes either Dirichlet

$$
\left.\phi^{A}\right|_{\partial M}=0
$$

or modified Neumann

$$
\left.\left(\nabla_{n} \phi^{A}+S^{A B} \phi^{B}\right)\right|_{\partial M}=0
$$

boundary conditions. $S^{A B}$ is an arbitrary symmetric matrix. Note, that the integral (3.7) vanishes also if $S^{A B}$ is an arbitrary symmetric (differential) operator on the boundary.

For the Dirichlet conditions (3.8) the boundary term in (3.2) vanishes automatically. To ensure absence of the surface term for the modified Neumann conditions (3.9) one should add to (3.1) an appropriate surface action.

\subsection{Bosonic string}

Our next example is the non-linear sigma model in two dimensions described by the action

$$
\begin{aligned}
\mathcal{L}^{[\sigma]}=\int_{M} & d^{2} x\left(\sqrt{g} G_{A B}(X) g^{\mu \nu} \partial_{\mu} X^{A} \partial_{\nu} X^{B}+\epsilon^{\mu \nu} B_{A B}(X) \partial_{\mu} X^{A} \partial_{\nu} X^{B}\right) \\
& +\int_{\partial M} A_{B} d X^{B}
\end{aligned}
$$

From the point of view of two-dimensional world sheet the fields $X^{A}(x)$ are scalars. In string theory they are interpreted as coordinates on a $d$-dimensional target manifold with the metric $G_{A B}(X) . \epsilon^{\mu \nu}$ is the Levi-Civita tensor density, $\epsilon^{12}=-\epsilon^{21}=1$. $B_{A B}(X)$ is an antisymmetric tensor field on the target space. $A_{B}(X)$ is the electromagnetic vector potential. The action (3.10) describes charged open stings. For simplicity, we absorb the inverse string tension $\alpha^{\prime}$ into a field redefinition. We do not include tachyon and dilaton couplings in the bulk or on the boundary.

Usually the term with the $B$-field gets an imaginary coefficient in the Euclidean space. Since the physical space-time has Minkowski signature it is not especially significant which way of continuation to the Euclidean space has been chosen provided the results are properly continued back to Minkowski space after the calculations. As we will see below, real coefficient in front of the $B$-field leads to a well-defined spectral problem. This situation is in close 
analogy with the continuation rules for the axial vector field in the spinor determinant $[14,13]$. The other way to deal with the field $B$ is to keep the coefficient of the $B$-term in (3.10) imaginary at the expense of introducing a more sophisticated conjugation operation [352] containing the sign-reversion of the $B$-field. The same refers to the electromagnetic potential $A_{B}$.

The field $X$ enters the action (3.10) at many places making the background field expansion a quite cumbersome procedure. The most economic way to arrange such an expansion and to calculate higher derivatives of the action (3.10) is to introduce the geodesic coordinates in the target space. A detailed explanation of the method as well as further references can be found in [87]. Consider the target space geodesics defined independently at each point of the two-dimensional world surface and parametrised by the arc length $s$ in the target space. They satisfy the usual geodesic equation

$$
\frac{d^{2}}{d s^{2}} X^{A}(x, s)+\gamma_{B C}^{A}(X) \frac{d}{d s} X^{B}(x, s) \frac{d}{d s} X^{C}(x, s)=0,
$$

where $\gamma_{B C}^{A}(X)$ is the Christoffel connection corresponding to the target space metric $G_{A B}$. Let us supplement the equation (3.11) by the initial conditions

$$
X^{A}(x, 0)=\bar{X}^{A}(x), \quad \frac{d}{d s} X^{A}(x, 0)=\xi^{A}(x),
$$

where $\bar{X}$ is the background field. $\xi^{A}$ parametrises deviations from $\bar{X}$ and, therefore, can be identified with quantum fluctuations. The $k$-th order term of the expansion of the action (3.10) around the background $\bar{X}$ is given by

$$
\mathcal{L}_{k}=\left.\frac{1}{k !} \frac{d^{k}}{d s^{k}} \mathcal{L}(X(s))\right|_{s=0}
$$

Higher order derivatives of $X(x, s)$ with respect to $s$ can be traded for the first derivatives by means of the geodesic equation (3.11) and then replaced by $\xi$ at $s=0$ with the help of the initial conditions (3.12).

It is convenient to introduce the Riemann curvature of the target space metric $G_{A B}, \mathcal{R}_{A B C D}$, and a 3 -index field strength

$$
H_{A B C}=\partial_{A} B_{B C}+\partial_{B} B_{C A}+\partial_{C} B_{B A}
$$

The covariant derivative $\nabla$ is

$$
\nabla_{\mu} \xi^{A}=\partial_{\mu} \xi^{A}+\gamma_{B C}^{A}(\bar{X})\left(\partial_{\mu} \bar{X}^{C}\right) \xi^{B}+\frac{1}{2} \epsilon_{\mu}{ }^{\nu}\left(\partial_{\nu} \bar{X}^{B}\right) H_{B C}^{A} \xi^{C}
$$

The quadratic part of the action (3.10) reads: 


$$
\begin{aligned}
\mathcal{L}_{2}= & \int_{M} d^{2} x \sqrt{h}\left(G_{A B}(\bar{X}) \nabla_{\mu} \xi^{A} \nabla^{\mu} \xi^{B}-\mathcal{R}_{A B C D}\left(\partial_{\mu} \bar{X}^{A}\right)\left(\partial^{\mu} \bar{X}^{D}\right) \xi^{B} \xi^{C}\right. \\
& -\frac{1}{2}\left(\partial_{\mu} \bar{X}^{C}\right)\left(\partial_{\nu} \bar{X}^{D}\right) \epsilon^{\mu \nu} \xi^{A} \xi^{B} D_{A} H_{B C D} \\
& \left.+\frac{1}{4}\left(\partial_{\mu} \bar{X}^{B}\right)\left(\partial^{\mu} \bar{X}^{D}\right) H_{A B C} H^{C}{ }_{D E} \xi^{A} \xi^{E}\right) \\
& +\int_{\partial M} d \tau\left(D_{\tau} \xi^{A} \xi^{B}\left(B_{A B}-F_{A B}\right)+\left(\partial_{\tau} \bar{X}^{B}\right) D_{A}\left(B_{B C}-F_{B C}\right) \xi^{A} \xi^{C}\right)
\end{aligned}
$$

where $\tau$ is the arc length along the boundary. $F_{B C}=\partial_{B} A_{C}-\partial_{C} A_{B}$. The covariant derivatives $D_{\tau}$ and $D_{A}$ contain the Christoffel connection on the target space (but not $H_{A B C}$ as the full covariant derivative $\nabla$, eq. (3.15)).

The natural inner product in the space of fluctuations $\xi$ reads

$$
<\xi_{(1)}, \xi_{(2)}>=\int_{M} d^{2} x \sqrt{g} G_{A B}(\bar{X}(x)) \xi_{(1)}^{A}(x) \xi_{(2)}^{B}(x) .
$$

The volume part of the action (3.16) has now the canonical form $\langle\xi, D \xi\rangle$ with the operator $D(2.2)$ which is obviously of Laplace type. The connection $\omega$ is defined in (3.15) and the endomorphism $E$ can be easily extracted from (3.16). The operator $D$ is formally self-adjoint with respect to (3.17) if we impose the boundary conditions of Neumann type

$$
\mathcal{B} \xi=\left.\left(\nabla_{n} \xi^{A}+\mathcal{S}_{B}^{A} \xi^{B}\right)\right|_{\partial M}=0
$$

with arbitrary operator $\mathcal{S}$ which should be symmetric with respect to the restriction of (3.17) to the boundary:

$$
\int_{\partial M} d \tau\left(\xi_{(1)}^{A} \mathcal{S}_{A}^{B} \xi_{(2) B}-\xi_{(2)}^{A} \mathcal{S}_{A}^{B} \xi_{(1) B}\right)=0
$$

There is a preferable choice of the boundary operator. Let us vary the action (3.16) with respect to the fluctuation field $\xi$ :

$$
\frac{1}{2} \delta S_{2}=\int_{M} d^{2} x \sqrt{h}(\delta \xi) D \xi-\int_{\partial M} d \tau(\delta \xi) \mathcal{B} \xi
$$

Now we require that the boundary integral in (3.20) vanishes for arbitrary $\delta \xi$. Hence we arrive at the boundary conditions (3.18) with the operator $\mathcal{S}$ given by

$$
\begin{aligned}
\mathcal{S}_{A}^{B}= & \frac{1}{2}\left(\Gamma \nabla_{\tau}+\nabla_{\tau} \Gamma\right)_{A}^{B}+S_{A}^{B}, \quad \Gamma_{A}^{B}=B_{A}^{B}-F_{A}{ }^{B} \\
S_{A}^{B}=\frac{1}{4}\left(\partial_{n} \bar{X}^{C}\right) & {\left[H^{D A}{ }_{C}\left(B_{D B}-F_{D B}\right)+H^{D}{ }_{B C}\left(B_{D}{ }^{A}-F_{D}{ }^{A}\right)\right] } \\
+ & \frac{1}{2}\left(\partial_{\tau} \bar{X}^{C}\right)\left[D^{A}\left(B_{B C}-F_{B C}\right)+D_{B}\left(B^{A}{ }_{C}-F^{A}{ }_{C}\right)\right] .
\end{aligned}
$$

Note that the operator in (3.21) is not of the ordinary Neumann (or Robin) type since it contains tangential derivatives on the boundary (cf. sec. 5.4.1). 
The variation (3.20) vanishes also if we choose Dirichlet boundary conditions for some of the coordinates of the string endpoints. Namely, we can take a projector $\Pi_{+}$and impose $(3.18)$ on $\left(\Pi_{+}\right)_{B}^{A} \xi^{b}$ and $\left.\left(1-\Pi_{+}\right)_{B}^{A} \delta \xi^{B}\right|_{\partial M}=0$. Physically this means that the endpoints of the bosonic string are confined to a submanifold in the target space. Such configurations [134] are called the Dirichlet branes.

\subsection{Spinor fields}

The action for the spinor fields $\psi$

$$
\mathcal{L}=\int_{M} d^{n} x \sqrt{g} \bar{\psi} \not D \psi
$$

contains a first order operator $\not D$ of Dirac type. In Euclidean space the conjugate spinor $\bar{\psi}$ is just the hermitian conjugate of $\psi: \bar{\psi}=\psi^{\dagger}$. By definition, an operator $\not D$ is of Dirac type if its square $D=\not D^{2}$ is of Laplace type. Spectral theory of general operators of Dirac type both on manifolds without boundaries and with local boundary conditions on manifolds with boundaries can be found in $[88,89]$. Here we consider some physically motivated particular cases only. Let us introduce the Euclidean Dirac $\gamma$-matrices which satisfy the Clifford commutation relations:

$$
\gamma_{\mu} \gamma_{\nu}+\gamma_{\nu} \gamma_{\nu}=2 g_{\mu \nu}
$$

The $\gamma$-matrices defined in this way are hermitian, $\gamma_{\mu}^{\dagger}=\gamma_{\mu}$. We also need the chirality matrix which will be denoted $\gamma^{5}$ independently of the dimension. It satisfies

$$
\left(\gamma^{5}\right)^{\dagger}=\gamma^{5}, \quad \gamma^{5} \gamma_{\mu}=-\gamma_{\mu} \gamma^{5}
$$

From now on we suppose that the dimension $n$ is even. We fix the sign in $\gamma^{5}$ by choosing

$$
\gamma^{5}=\frac{i^{\frac{n(n-1)}{2}}}{n !} \epsilon^{\mu \ldots \nu} \gamma_{\mu} \ldots \gamma_{\nu}
$$

Let $\not D$ be the standard Dirac operator in curved space with gauge and axial gauge connection

$$
\not D=i \gamma^{\mu}\left(\partial_{\mu}+\frac{1}{8}\left[\gamma_{\nu}, \gamma_{\rho}\right] \sigma_{\mu}^{\nu \rho}+A_{\mu}+i A_{\mu}^{5} \gamma^{5}\right)
$$

Here $\sigma_{\mu}^{\nu \rho}$ is the spin-connection (2.9). $A_{\mu}$ and $A_{\mu}^{5}$ are vector and axial vector fields respectively taken in some representation of the gauge group. Both $A_{\mu}$ and $A_{\mu}^{5}$ are antihermitian in the gauge indices. The operator $\not D(3.26)$ is formally self-adjoint in the bulk. The operator $D=\not D^{2}$ is of Laplace type $(2.2)$ with 10

\footnotetext{
10 The present author is grateful to Valery Marachevsky for his help in deriving and checking eqs. (3.27), (3.28) and (3.37), see also [136].
} 


$$
\begin{gathered}
\omega_{\mu}=\frac{1}{8}\left[\gamma_{\nu}, \gamma_{\rho}\right] \sigma_{\mu}^{\nu \rho}+A_{\mu}+\frac{i}{2}\left[\gamma_{\mu}, \gamma_{\nu}\right] A^{5 \nu} \gamma^{5} \\
E=-\frac{1}{4} R+\frac{1}{4}\left[\gamma^{\mu}, \gamma^{\nu}\right] F_{\mu \nu}+i \gamma^{5} D^{\mu} A_{\mu}^{5}-(n-2) A_{\mu}^{5} A^{5 \mu} \\
-\frac{1}{4}(n-3)\left[\gamma^{\mu}, \gamma^{\nu}\right]\left[A_{\mu}^{5}, A_{\nu}^{5}\right]
\end{gathered}
$$

with obvious notations $F_{\mu \nu}=\partial_{\mu} A_{\nu}-\partial_{\nu} A_{\mu}+\left[A_{\mu}, A_{\nu}\right], D_{\mu} A_{\nu}^{5}=\partial_{\mu} A_{\nu}^{5}-\Gamma_{\mu \nu}^{\rho} A_{\rho}^{5}+$ $\left[A_{\mu}, A_{\nu}^{5}\right]$. The expression for $\Omega_{\mu \nu}$ is a little bit lengthy:

$$
\begin{aligned}
\Omega_{\mu \nu}= & F_{\mu \nu}-\left[A_{\mu}^{5}, A_{\nu}^{5}\right]-\frac{1}{4} \gamma^{\sigma} \gamma^{\rho} R_{\sigma \rho \mu \nu}-i \gamma^{5} \gamma^{\rho}\left(\gamma_{\nu} D_{\mu} A_{\rho}^{5}-\gamma_{\mu} D_{\nu} A_{\rho}^{5}\right) \\
& +i \gamma^{5} A_{\mu \nu}^{5}+\left[A_{\mu}^{5}, A_{\rho}^{5}\right] \gamma^{\rho} \gamma_{\nu}-\left[A_{\nu}^{5}, A_{\rho}^{5}\right] \gamma^{\rho} \gamma_{\mu} \\
& -\gamma^{\rho} A_{\rho}^{5} \gamma_{\mu} \gamma^{\sigma} A_{\sigma}^{5} \gamma_{\nu}+\gamma^{\rho} A_{\rho}^{5} \gamma_{\nu} \gamma^{\sigma} A_{\sigma}^{5} \gamma_{\mu}
\end{aligned}
$$

where

$$
A_{\mu \nu}^{5}=\partial_{\mu} A_{\nu}^{5}-\partial_{\nu} A_{\mu}^{5}+\left[A_{\mu}, A_{\nu}^{5}\right]-\left[A_{\nu}, A_{\mu}^{5}\right]
$$

Consider now manifolds with boundary. The specific feature of the action (3.22) is that it contains first order derivatives only. Consequently, boundary conditions should be imposed on a half of the spinor components. Let these be Dirichlet boundary conditions

$$
\left.\Pi_{-} \psi\right|_{\partial M}=0
$$

where $\Pi_{-}$is a hermitian projector, $\Pi_{-}^{2}=\Pi_{-}, \Pi_{-}^{\dagger}=\Pi_{-}$. Due to the hermiticity

$$
\left.\bar{\psi} \Pi_{-}\right|_{\partial M}=0
$$

Following Luckock [308] let us consider a family of the projectors

$$
\Pi_{-}=\frac{1}{2}\left(1+\gamma^{n} \exp \left(i q \gamma^{5}\right)\right)
$$

where $q$ is a scalar which can depend on the coordinate on the boundary.

To make the operator $\not D$ formally self-adjoint including the boundary we must require that

$$
\int_{\partial M} d^{n-1} x \sqrt{h} \bar{\psi}_{1} \gamma_{n} \psi_{2}=0
$$

for all $\psi_{1,2}$ satisfying the boundary conditions (3.30). Since $\psi_{1}$ and $\psi_{2}$ are arbitrary, the projector $\Pi_{-}$should satisfy

$$
\left(1-\Pi_{-}\right) \gamma^{n}\left(1-\Pi_{-}\right)=0
$$

This condition yields $q= \pm \pi / 2$. The projector (3.32) takes the form

$$
\Pi_{-}=\frac{1}{2}\left(1 \pm i \gamma^{n} \gamma^{5}\right)
$$


To formulate the spectral problem for the second order operator $D=\not D^{2}$ we need boundary conditions for the second half of the spinor components. The relevant functional space should be spanned by the eigenfunctions of the Dirac operator $\not D$. It is clear that on this space the functions $\not D \psi$ should satisfy the same boundary conditions (3.30) as the $\psi$ 's themselves:

$$
\left.\Pi_{-} \not D \psi\right|_{\partial M}=0 \text {. }
$$

Let us adopt the choice (3.35) for $\Pi_{-}$. By commuting $\Pi_{-}$with $\not D$ in (3.36) we obtain

$$
\left.\left(\nabla_{n}+S\right) \Pi_{+} \psi\right|_{\partial M}=0, \quad S=-\frac{1}{2} L_{a a} \Pi_{+}, \quad \Pi_{+}=1-\Pi_{-} .
$$

We remind that $L_{a b}$ is extrinsic curvature of the boundary. The boundary conditions (3.30), (3.37) with (3.35) are mixed (cf. (2.17)). Spectral geometry of the Dirac operator with these boundary conditions has been thoroughly studied by Branson and Gilkey [89].

We can generalise the boundary conditions presented above by considering non-hermitian projectors, $\Pi_{-}^{\dagger} \neq \Pi_{-}$. Then the boundary condition for the conjugated spinors reads:

$$
\left.\bar{\psi} \Pi_{-}^{\dagger}\right|_{\partial M}=0 .
$$

Instead of (3.34) we have the condition

$$
\left(1-\Pi_{-}^{\dagger}\right) \gamma^{n}\left(1-\Pi_{-}\right)=0,
$$

which yields

$$
\Pi_{-}=\frac{1}{2}\left(1+i \gamma^{n} \gamma^{5} e^{r\left(x^{a}\right) \gamma^{5}}\right)
$$

with an arbitrary real function (or even with an arbitrary hermitian matrix valued function) $r\left(x^{a}\right)$.

In the Minkowski signature space the spinor conjugation includes $\gamma^{0}$. Therefore, the boundary conditions on the conjugate spinor (3.31) are changed. Roughly speaking, to continue $\Pi_{-}$to the Minkowski signature space time one has to replace $\gamma^{n} \gamma^{5}$ by $\gamma^{n}$ in (3.35) and (3.40) and to take into account powers of $i$ which appear in the Dirac gamma matrices.

In particle physics, interest to the boundary conditions defined by (3.35) is due to the MIT bag model of hadrons proposed in [124,125,143]. This model was modified later $[126,111,95,397,365,250]$ to include a chiral phase on the boundary in a manner similar to (3.40) (for a review see [273]). Renormalization of quantum field theory with the boundary conditions defined by (3.35) was considered by Symanzik [394].

As we have already mentioned above, bag boundary conditions defined by the projector (3.35) are a particular case of mixed boundary conditions (2.17). They will be considered in detail in sec. 5.3 (see also sec. 5.1 for further references to calculations in a ball). Chiral bag boundary conditions defined 
by (3.40) with $r \neq 0$ are considerably more complicated because the equation (3.36) contains a mixture of normal and tangential derivatives. We refer to $[281,203,204,138,421,139,137,62,199]$ for more calculations of spectral functions in this latter case.

The boundary conditions considered in this section are local, i.e. they treat the fields at each point of the boundary independently. One can also define global boundary conditions for the Dirac operator (cf. sec. 5.4.2).

Due to the fermionic nature of the spinor field the path integral over $\psi$ and $\bar{\psi}$ gives determinant of the operator $\not D$ to a positive power,

$$
Z=\int \mathcal{D} \bar{\psi} \mathcal{D} \psi \exp (-\mathcal{L}(\bar{\psi}, \psi))=\operatorname{det} \not D
$$

where the action $\mathcal{L}$ is given by (3.22) and $\bar{\psi}, \psi$ are complex Dirac spinors.

\subsection{Vector fields}

Consider the Yang-Mills action for the gauge field $A_{\mu}^{\alpha}$ (Greek letters from the beginning of the alphabet label generators of the gauge group):

$$
\mathcal{L}=\frac{1}{4} \int_{M} d^{n} x \sqrt{g} F_{\mu \nu}^{\alpha} F^{\mu \nu \alpha}
$$

where, as usual, $F_{\mu \nu}^{\alpha}=\partial_{\mu} A_{\nu}^{\alpha}-\partial_{\nu} A_{\mu}^{\alpha}+c_{\beta \gamma}^{\alpha} A_{\mu}^{\beta} A_{\nu}^{\gamma} \cdot c_{\beta \gamma}^{\alpha}$ are totally antisymmetric structure constants of the gauge group. Let us introduce the background field $B_{\mu}^{\alpha}$ by the shift $A_{\mu}^{\alpha} \rightarrow B_{\mu}^{\alpha}+A_{\mu}^{\alpha}$. From now on $A_{\mu}^{\alpha}$ plays the role of quantum fluctuations. The quadratic part of the action reads:

$$
\begin{aligned}
\mathcal{L}_{2}=\frac{1}{2} \int_{M} d^{n} x \sqrt{g}[ & -A^{\alpha \nu} \nabla^{\mu} \nabla_{\mu} A_{\nu}^{\alpha}+A_{\mu}^{\alpha} \nabla^{\mu} \nabla^{\nu} A_{\nu}^{\alpha}+A_{\nu}^{\alpha} A_{\mu}^{\alpha} R^{\mu \nu} \\
& \left.+2 F_{\mu \nu}^{\alpha}(B) c_{\beta \gamma}^{\alpha} A^{\beta \mu} A^{\gamma \nu}\right] \\
+ & \frac{1}{2} \int_{\partial M} d^{n-1} x \sqrt{h}\left[A_{\nu}^{\alpha} \nabla^{\nu} A_{n}^{\alpha}-A^{\alpha \nu} \nabla_{n} A_{\nu}^{\alpha}\right] .
\end{aligned}
$$

The covariant derivative $\nabla$ contains both metric and gauge parts: $\nabla_{\nu} A_{\mu}^{\alpha}=$ $\partial_{\nu} A_{\mu}^{\alpha}+B_{\nu}^{\beta} c_{\beta \gamma}^{\alpha} A_{\nu}^{\gamma}-\Gamma_{\nu \mu}^{\rho} A_{\rho}^{\alpha}$. One should impose a gauge condition on the fluctuation $A_{\mu}^{\alpha}$. We choose

$$
\nabla^{\mu} A_{\mu}^{\alpha}=0
$$

In the gauge (3.44) the bulk term in (3.43) defines an elliptic operator of the Laplace type with

$$
\begin{aligned}
& \left(\omega_{\mu}\right)_{\nu \beta}^{\alpha \rho}=B_{\mu}^{\gamma} c_{\gamma \beta}^{\alpha} \delta_{\nu}^{\rho}-\Gamma_{\mu \nu}^{\rho} \delta_{\beta}^{\alpha}, \\
& (E)_{\nu \beta}^{\alpha \rho}=-R_{\nu}^{\rho} \delta_{\beta}^{\alpha}+2 F(B)^{\gamma \rho}{ }_{\nu} c_{\beta \alpha}^{\gamma} .
\end{aligned}
$$

The field strength corresponding of the connection (3.45) reads

$$
\left(\Omega_{\mu \nu}\right)_{\rho \beta}^{\alpha \sigma}=R_{\rho \mu \nu}^{\sigma} \delta_{\beta}^{\alpha}+F(B)_{\mu \nu}^{\delta} c_{\delta \beta}^{\alpha} \delta_{\rho}^{\sigma}
$$


Note, that all the quantities above (3.45) - (3.47) are matrix functions with both gauge and vector indices.

On a manifold without boundary the operator $D$ with (3.45) and (3.46) is symmetric with respect to the standard inner product in the space of the vector fields

$$
<A^{(1)}, A^{(2)}>=\int_{M} \sqrt{g} d^{n} x A^{(1) \alpha \mu} A_{\mu}^{(2) \alpha} .
$$

The ghost operator corresponding to the gauge (3.44) is just the ordinary scalar Laplacian, $D^{g h}=-\nabla^{[g h] \mu} \nabla_{\mu}^{[g h]}$, with the connection

$$
\left(\omega_{\mu}^{[g h]}\right)_{\gamma}^{\alpha}=B_{\mu}^{\beta} c_{\beta \gamma}^{\alpha}
$$

The one-loop path integral reads:

$$
Z(B)=\operatorname{det}(D)_{\perp}^{-\frac{1}{2}} \operatorname{det}\left(D^{[g h]}\right)^{\frac{1}{2}}
$$

where the first determinant is restricted to the fields satisfying the gauge condition (3.44). Note, that pure gauge fields $A=\nabla \xi$ are zero modes of the total operator $D+\nabla \nabla$ of the bulk action (3.43) only on shell, i.e. when the background field $B$ satisfies the classical equation of motion $\nabla^{\mu} F(B)_{\mu \nu}^{\alpha}=0$. Therefore, the path integral is gauge-independent on shell, but it depends on the gauge choice off shell. For example, the Feynman gauge path integral

$$
Z_{F}(B)=\operatorname{det}(D)^{-\frac{1}{2}} \operatorname{det}\left(D^{[g h]}\right),
$$

where the first determinant is calculated on the space of all vector field, is equal to the $Z(B)$ defined in (3.50) only on shell. However, physical predictions of the two path integrals are, of course, equivalent.

The path integral (3.51) can be obtained by adding the gauge fixing term

$$
\mathcal{L}_{\text {gf }}=\frac{1}{2 \kappa} \int_{M} d^{n} x \sqrt{g}\left(\nabla_{\mu} A^{\mu}\right)^{2}
$$

to $(3.43)$ with $\kappa=1$. The case $\kappa \neq 1$ yields a non-minimal operator on the gauge field fluctuations (cf sec. 4.4).

One can define "total" heat kernel coefficients for the path integral in a certain gauge $\chi(A)$

$$
a_{k}^{t o t}=a_{k}\left(D^{\chi}\right)-2 a_{k}\left(D_{\chi}^{[g h]}\right)
$$

where $D^{\chi}$ is defined by the action (3.43) with the gauge fixing term $\chi^{2}(A)$, and the ghost operator is $D_{\chi}^{[g h]} \xi=\chi(\nabla \xi)$. Even on shell only the coefficient $a_{n}^{\text {tot }}$ is gauge-independent. Only this coefficient contains information on the one-loop divergences in a gauge-invariant regularization like the $\zeta$-function one.

On a manifold with boundary one should impose boundary conditions on gauge fields and ghosts. The boundary conditions should be gauge invariant. Consider a more general set-up when we have some quantum fields $\Phi$ and a linearised gauge transformation $\Phi \rightarrow \Phi+\delta_{\xi} \Phi$ with local parameter $\xi$. 
Boundary operator $\mathcal{B}$ defines gauge invariant boundary conditions

$$
\mathcal{B} \Phi=0
$$

if there exist boundary conditions for the gauge parameter $\xi$

$$
\mathcal{B}_{\xi} \xi=0
$$

such that

$$
\mathcal{B} \delta_{\xi} \Phi=0 .
$$

The equation (3.56) means that the functional space defined by the boundary conditions (3.54) is invariant under the gauge transformations provided the gauge parameter $\xi$ satisfies (3.55). Upon quantisation (3.55) become boundary conditions for the ghost fields. The condition (3.56) ensures validity of the Faddeev-Popov trick on a manifold with boundary and guarantees gaugeindependence of the on-shell path integral [405].

Since the boundary term in (3.43) is diagonal in the gauge index $\alpha$ we can consider the case of an abelian gauge group and drop $\alpha$ from the notations. The general non-abelian case does not offer considerable complications. There are two sets of local boundary conditions which satisfy the gauge-invariance requirements above. The first set is called absolute boundary conditions. It reads

$$
\begin{aligned}
& \left.A_{n}\right|_{\partial M}=0,\left.\quad \partial_{n} A_{a}\right|_{\partial M}=\left.\left(\nabla_{n} \delta_{a b}-L_{a b}\right) A_{b}\right|_{\partial M}=0 \\
& \left.\nabla_{n} \xi\right|_{\partial M}=0 .
\end{aligned}
$$

The second set

$$
\begin{aligned}
& \left.\left(\nabla_{n}-L_{a a}\right) A_{n}\right|_{\partial M}=0,\left.\quad A_{a}\right|_{\partial M}=0, \\
& \left.\xi\right|_{\partial M}=0
\end{aligned}
$$

is called relative boundary conditions. The projectors on the Dirichlet and Neumann subspaces and the endomorphism $S$ are $\left(\Pi^{-}\right)_{i j}=\delta_{i n} \delta_{j n},\left(\Pi^{+}\right)_{i j}=$ $\delta_{i j}-\delta_{i n} \delta_{j n}, S_{i j}=-L_{a b} \delta_{i a} \delta_{j b}$ and $\left(\Pi^{-}\right)_{i j}=\delta_{i j}-\delta_{i n} \delta_{j n},\left(\Pi^{+}\right)_{i j}=\delta_{i n} \delta_{j n}, S_{i j}=$ $-L_{a a} \delta_{i n} \delta_{j n}$ for the absolute and relative boundary conditions respectively.

It is straightforward to check gauge invariance of the absolute boundary conditions (3.57). A bit more job is required to show that the condition (3.58) for the normal component is also gauge invariant. The key observation is that near the boundary the scalar Laplacian can be represented as $D^{[g h]}=$ $-\left(\nabla_{n}-L_{a a}\right) \nabla_{n}+\mathcal{E}$, where $\mathcal{E}$ does not contain normal derivatives and, therefore, $\left.\mathcal{E} \xi\right|_{\partial M}=0$ under the boundary conditions (3.58) for the $\xi$. Consequently,

$$
\left.\left(\nabla_{n}-L_{a a}\right) \delta_{\xi} A_{n}\right|_{\partial M}=\left.\left(\nabla_{n}-L_{a a}\right) \nabla_{n} \xi\right|_{\partial M}=\left.\left(-D^{[g h]} \xi+\mathcal{E} \xi\right)\right|_{\partial M}=0
$$

where $\left.D^{[g h]} \xi\right|_{\partial M}=0$ on the eigenfunctions of the operator $D^{[g h]}$ which is enough for our purposes. 
The boundary term in the action $(3.43)$ can be rewritten as ${ }^{11}$

$$
\frac{1}{2} \int_{\partial M} d^{n-1} x \sqrt{h}\left[A_{a}\left(A_{n: a}+L_{a b} A_{b}-A_{a ; n}\right)\right] .
$$

This boundary action vanishes for both types of the boundary conditions (3.57) and (3.58). Hence the operator $D$ is symmetric for these boundary conditions. Another remarkable fact is that the fields $A^{\perp}$ satisfying the gauge condition (3.44) are orthogonal to the gauge transformations. Indeed,

$$
<A^{\perp}, \nabla \xi>=-\int_{\partial M} d^{n-1} x \sqrt{h} A_{n}^{\perp} \xi=0
$$

for both (3.57) and (3.58).

We should also check whether the gauge condition (3.44) is indeed compatible with the boundary conditions (3.58) and (3.57). For an arbitrary $A$ there should exist unique $\xi$ such that

$$
\nabla^{\mu}\left(A_{\mu}+\nabla_{\mu} \xi\right)=0
$$

We rewrite $(3.62)$ as

$$
\nabla^{\mu} A_{\mu}=-\Delta \xi
$$

Let us start with relative boundary conditions (3.58). In this case the left hand side of (3.63) satisfies Dirichlet boundary conditions. The scalar Laplacian $\Delta$ for these boundary conditions is invertible. Therefore, a solution for (3.63) always exists, and it is unique. The case of absolute boundary conditions is a bit more involved. One should take care of a zero mode in the ghost sector. We leave this case as an exercise. A more extensive discussion of compatibility of gauge and boundary conditions can be found in [405].

\subsection{Graviton}

We start this section with the Einstein-Hilbert action on a four dimensional Euclidean manifold without boundary:

$$
\mathcal{L}=\frac{1}{16 \pi G_{N}} \int d^{4} x \sqrt{g}(R-2 \Lambda)
$$

where $R$ is the scalar curvature, $G_{N}$ is the Newton constant, $\Lambda$ is the cosmological constant. As usual, let us shift the metric $g_{\mu \nu} \rightarrow g_{\mu \nu}+h_{\mu \nu}$. From now on $g_{\mu \nu}$ will denote the background metric, $h_{\mu \nu}$ will be the quantum fluctuations. We can decompose the $h_{\mu \nu}$ further in trace, longitudinal and transverse-traceless part:

$$
h_{\mu \nu}=\frac{1}{4} h g_{\mu \nu}+(L \xi)_{\mu \nu}+h_{\mu \nu}^{\perp},
$$

$\overline{11}$ The equation to follow contains two types of covariant derivatives. Definitions can be found in sec. 2.1 . 
where $g^{\mu \nu} h_{\mu \nu}^{\perp}=\nabla^{\nu} h_{\mu \nu}^{\perp}=0$ and

$$
(L \xi)_{\mu \nu}=\nabla_{\mu} \xi_{\nu}+\nabla_{\nu} \xi_{\nu}-\frac{1}{2} g_{\mu \nu} \nabla^{\rho} \xi_{\rho}
$$

The decomposition (3.65) is orthogonal with respect to the inner product:

$$
\begin{aligned}
& <h, h^{\prime}>=\int d^{4} x \sqrt{g} G^{\mu \nu \rho \sigma} h_{\mu \nu} h_{\rho \sigma}^{\prime} \\
& G^{\mu \nu \rho \sigma}=\frac{1}{2}\left(g^{\mu \rho} g^{\nu \sigma}+g^{\mu \sigma} g^{\nu \rho}+C g^{\mu \nu} g^{\rho \sigma}\right) .
\end{aligned}
$$

Here $C$ is a constant. For positivity of (3.67) $C$ should be greater than $-\frac{1}{2}$. Under the action of infinitesimal diffeomorphism generated by a vector $\epsilon_{\mu}$ the components of (3.65) transform as

$$
\xi_{\mu} \rightarrow \xi_{\mu}+\epsilon_{\mu}, \quad h \rightarrow h+2 \nabla^{\mu} \epsilon_{\mu}, \quad h_{\mu \nu}^{\perp} \rightarrow h_{\mu \nu}^{\perp}
$$

One can fix the gauge freedom (3.68) by the condition

$$
\xi_{\mu}=0
$$

If the background admits conformal Killing vectors (these are the vectors which are annihilated by the operator $L$ (3.66)) the condition (3.69) is not enough and one should impose one more gauge condition on the trace part (see e.g. [402]). We suppose that conformal Killing vectors are absent.

The Jacobian factor induced by the change of variables $(3.65) h_{\mu \nu} \rightarrow$ $\left(h, \xi_{\mu}, h_{\mu \nu}^{\perp}\right)$ is

$$
J=\operatorname{det}_{V}\left(L^{\dagger} L\right)^{\frac{1}{2}}
$$

where the determinant is calculated on the space of the vector fields (excluding the conformal Killing vectors which we do not take into account). It is convenient to shift the scalar part of the metric fluctuations by $\nabla^{\mu} \xi_{\mu}$ so that the decomposition (3.65) becomes

$$
h_{\mu \nu}=\frac{1}{4}\left(\sigma+2 \nabla^{\rho} \xi_{\rho}\right) g_{\mu \nu}+(L \xi)_{\mu \nu}+h_{\mu \nu}^{\perp} .
$$

Since the change of the variables $h \rightarrow \sigma$ does not introduce any Jacobian factor we conclude that the path integral measure is

$$
\mathcal{D} h_{\mu \nu}=\operatorname{det}_{V}\left(L^{\dagger} L\right)^{\frac{1}{2}} \mathcal{D} \sigma \mathcal{D} \xi \mathcal{D} h^{\perp} .
$$

To simplify the discussion we suppose that the background metric $g_{\mu \nu}$ satisfies the Einstein equations

$$
R_{\mu \nu}(g)=\Lambda g_{\mu \nu}
$$

The quadratic part of the action reads: 


$$
\begin{aligned}
\mathcal{L}_{2}=\frac{1}{16 \pi G} \int & d^{4} x \sqrt{g}\left(\frac{1}{4} h^{\perp \mu \nu}\left(-\Delta g_{\mu \rho} g_{\nu \sigma}+2 R_{\mu \rho \nu \sigma}\right) h^{\perp \rho \sigma}\right. \\
& \left.-\frac{3}{32} \sigma\left(-\Delta-\frac{R}{3}\right) \sigma\right) .
\end{aligned}
$$

Due to the gauge invariance (3.74) does not contain $\xi$. Functional integration over $\xi$ produces an infinite constant equivalent to the volume of the diffeomorphism group which will be neglected.

The kinetic term for $\sigma$ has a wrong (negative) sign. This represents the well known conformal factor problem of quantum general relativity. Different explanations of this phenomenon $[232,375,314,348]$ suggest (roughly) the same remedy: the conformal mode must be rotated to the imaginary axis, $\sigma \rightarrow i \sigma$.

The path integral can be written in terms of functional determinants corresponding to vector fields (indicated by the subscript $V$ ), scalars (indicated by $S$ ), and transverse traceless tensors (indicated by $T \perp$ ):

$$
Z=\operatorname{det}_{V}\left(L^{\dagger} L\right)^{\frac{1}{2}} \operatorname{det}_{S}\left(-\Delta-\frac{R}{3}\right)^{-\frac{1}{2}} \operatorname{det}_{T \perp}\left(-\Delta g_{\mu \rho} g_{\nu \sigma}+2 R_{\mu \rho \nu \sigma}\right)^{-\frac{1}{2}} .
$$

This expression is, in principle, suitable for calculations on some homogeneous spaces since the harmonic expansion on such spaces usually respects separation of tensors to transverse and longitudinal parts. However, we must warn the reader again that we have neglected the presence of Killing vectors and of conformal Killing vectors. The ways to treat these vectors created a long discussion in the literature [233, 128, 211, 8, 254, 396, 402, 335, 415].

The representation (3.75) is not convenient on generic manifold since the tensor operator is restricted to the transverse modes. To remove this restriction one can either multiply (3.75) by a compensating vector determinant, or add a suitable gauge fixing term to the classical action (3.64) and repeat the quantisation procedure right from the beginning. The result can be found elsewhere in the literature (see, e.g., [233, 128,211,396]).

Boundary conditions for one-loop Euclidean quantum gravity must be diffeomorphism invariant and must lead to "good" (i.e. symmetric and elliptic) operators describing metric and ghost fluctuations. The problem of finding a suitable set of boundary conditions in gravity appeared to be much harder that for the lower spin fields. There exist several proposals on the market $[47,308,193,412,313,39,38,36,334,190]$. However, neither of these proposals is fully satisfactory. It is clear now that proper boundary conditions must depend on tangential derivatives. Moreover, they probably must be non-local.

The material of this section can be generalised to the case when an independent torsion field is present. Explicit expressions for relevant operators acting on fields with different spin in the Riemann-Cartan space can be found in $[350,132,426]$. 


\section{Heat kernel expansion on manifolds without boundary}

\subsection{General formulae}

For physicists, the most familiar way to calculate the heat kernel coefficients is the DeWitt iterative procedure which will be briefly described in sec. 4.3. Here we take a different route following the method of Gilkey. Advantages of this method are most clearly seen on manifolds with boundaries. However, even if boundaries are absent we use the same method for the following reasons: (i) this will ensure a smooth transition to more complicated material of the next section; (ii) we believe that calculations of the coefficients $a_{k}(f, D)$ are a little bit easier even without a boundary. We are not going to give complete proofs of all statements. Instead, we concentrate on main ideas of the method. For the details an interested reader can consult the original paper [244] and the monographs [245,297].

We start with very general properties of the heat kernel coefficients. Let us consider a smooth compact Riemannian manifold $M$ without boundary. To be able to define functions on $M$ which carry some discrete (spin or gauge) indices we need a vector bundle $V$ over $M$. Let $D$ be an operator of Laplace type on $V$, and let $f$ be a smooth function on $M$. There is an asymptotic expansion (2.21) and

1) Coefficients with odd index $k$ vanish, $a_{2 j+1}(f, D)=0$;

2) Coefficients $a_{2 j}(f, D)$ are locally computable in terms of geometric invariants.

Already the existence of the power-law asymptotic expansion (2.21) is a non-trivial statement. We postpone the discussion of this property to sec. 5.4.

The second statement above is very important. It means that the heat kernel coefficients can be expressed as integrals of local invariants:

$$
a_{k}(f, D)=\operatorname{tr}_{V} \int_{M} d^{n} x \sqrt{g}\left\{f(x) a_{k}(x ; D)\right\}=\sum_{I} \operatorname{tr}_{V} \int_{M} d^{n} x \sqrt{g}\left\{f u^{I} \mathcal{A}_{k}^{I}(D)\right\}
$$

where $\mathcal{A}_{k}^{I}$ are all possible independent invariants of the dimension $k$ constructed from $E, \Omega, R_{\mu \nu \rho \sigma}$ and their derivatives. We use usual assignments of the dimensions when $E$ has dimension two, any derivative has dimension one, etc. $u^{I}$ are some constants. For example, if $k=2$ only two independent invariants exist. These are $E$ and $R$. Note, that we can always integrate by parts to remove all derivatives from $f$. The first statement, $a_{2 j+1}=0$, is clear now. One cannot construct an odd-dimension invariant on a manifold without boundary.

Let us study further relations between the heat kernel coefficients which will turn out to generate relations between the constants $u^{I}$. Consider now the case when the manifold $M$ is a direct product of two manifolds, $M_{1}$ and $M_{2}$, with coordinates $x_{1}$ and $x_{2}$ respectively, and the operator $D$ is a sum of two operators acting independently on $M_{1}$ and $M_{2}, D=D_{1} \otimes 1+1 \otimes D_{2}$. 
This means that the bundle indices are also independent. As an example, one can consider the vector Laplacian on $M_{1} \times M_{2}$. One can write symbolically $\exp (-t D)=\exp \left(-t D_{1}\right) \otimes \exp \left(-t D_{2}\right)$. Next we multiply both sides of this equation by $f\left(x_{1}, x_{2}\right)=f_{1}\left(x_{1}\right) f_{2}\left(x_{2}\right)$, take the functional trace and perform the asymptotic expansion in $t$ to get

$$
a_{k}(x ; D)=\sum_{p+q=k} a_{p}\left(x_{1} ; D_{1}\right) a_{q}\left(x_{2} ; D_{2}\right) .
$$

The consequences of eq. (4.2) are very far reaching. In particular, eq. (4.2) allows to fix the dependence of $u^{I}$ on the dimension of the manifold $M$. Consider an even more specialised case when one of the manifolds is a onedimensional circle: $M_{1}=S^{1}, 0<x_{1} \leq 2 \pi$. Let us make the simplest choice for $D_{1}: D_{1}=-\partial_{x_{1}}^{2}$. All geometric invariants associated with $D$ are defined solely by the $D_{2}$-part. Moreover, all invariants are independent of $x_{1}$. Therefore, by the equation (4.1),

$$
\begin{aligned}
a_{k}\left(f\left(x_{2}\right), D\right) & =\int_{S^{1} \times M_{2}} d^{n} x \sqrt{g} \sum_{I} \operatorname{tr}_{V}\left\{f\left(x_{2}\right) u_{(n)}^{I} \mathcal{A}_{k}^{I}(D)\right\} \\
& =2 \pi \int_{M_{2}} d^{n-1} x \sqrt{g} \sum_{I} \operatorname{tr}_{V}\left\{f\left(x_{2}\right) u_{(n)}^{I} \mathcal{A}_{k}^{I}\left(D_{2}\right)\right\}
\end{aligned}
$$

Here dependence of the constants $u_{(n)}^{I}$ on the dimension $n$ of the manifold is shown explicitly. On the other hand, we can use (4.2). Spectrum of the operator $D_{1}$ is known. The eigenvalues are $l^{2}, l \in \mathbb{Z}$. The heat kernel asymptotics for $D_{1}$ can be easily obtained by using the Poisson summation formula

$$
\begin{aligned}
K\left(t, D_{1}\right) & =\sum_{l \in \mathbb{Z}} \exp \left(-t l^{2}\right)=\sqrt{\frac{\pi}{t}} \sum_{l \in \mathbb{Z}} \exp \left(-\pi^{2} l^{2} / t\right) \\
& \simeq \sqrt{\frac{\pi}{t}}+\mathcal{O}\left(e^{-1 / t}\right) .
\end{aligned}
$$

Since exponentially small terms have no effect on the heat kernel coefficients, the only non-zero coefficient is $a_{0}\left(1, D_{1}\right)=\sqrt{\pi}$. Therefore, we obtain by eq. $(4.2)$

$$
a_{k}\left(f\left(x^{2}\right), D\right)=\sqrt{\pi} \int_{M_{2}} d^{n-1} x \sqrt{g} \sum_{I} \operatorname{tr}_{V}\left\{f\left(x^{2}\right) u_{(n-1)}^{I} \mathcal{A}_{n}^{I}\left(D_{2}\right)\right\}
$$

There are no restrictions on the operator $D_{2}$ or on the manifold $M_{2}$. By comparing the equations (4.3) and (4.5) we obtain

$$
u_{(n)}^{I}=\sqrt{4 \pi} u_{(n+1)}^{I} .
$$

This proves that the constants $u^{I}$ depend on the dimension $n$ only through the overall normalisation factor $(4 \pi)^{-n / 2}$.

To calculate the heat kernel coefficients we need also the following variational equations (see [245], Lemma 4.1.15): 


$$
\begin{aligned}
& \left.\frac{d}{d \epsilon}\right|_{\epsilon=0} a_{k}\left(1, e^{-2 \epsilon f} D\right)=(n-k) a_{k}(f, D), \\
& \left.\frac{d}{d \epsilon}\right|_{\epsilon=0} a_{k}(1, D-\epsilon F)=a_{k-2}(F, D), \\
& \left.\frac{d}{d \epsilon}\right|_{\epsilon=0} a_{n-2}\left(e^{-2 \epsilon f} F, e^{-2 \epsilon f} D\right)=0,
\end{aligned}
$$

where $f$ and $F$ are some smooth functions.

To prove the first property (4.7) we note that

$$
\left.\frac{d}{d \epsilon}\right|_{\epsilon=0} \operatorname{Tr}\left(\exp \left(-e^{-2 \epsilon f} t D\right)\right)=\operatorname{Tr}(2 f t D \exp (-t D))=-2 t \frac{d}{d t} \operatorname{Tr}(f \exp (-t D))
$$

and expand both sides of this equation in the power series in $t$. Eq. (4.8) can be checked in a similar way. To prove (4.9) consider the operator

$$
D(\epsilon, \delta)=e^{-2 \epsilon f}(D-\delta F)
$$

We use first (4.7) with $k=n$ to show:

$$
0=\left.\frac{d}{d \epsilon}\right|_{\epsilon=0} a_{n}(1, D(\epsilon, \delta))
$$

Then we vary the equation above with respect to $\delta$ :

$$
0=\left.\left.\frac{d}{d \delta}\right|_{\delta=0} \frac{d}{d \epsilon}\right|_{\epsilon=0} a_{n}(1, D(\epsilon, \delta))=\left.\left.\frac{d}{d \epsilon}\right|_{\epsilon=0} \frac{d}{d \delta}\right|_{\delta=0} a_{n}(1, D(\epsilon, \delta)) .
$$

Finally, eq. (4.8) yields (4.9).

Eq. (4.8) restricts dependence of the heat kernel coefficients on the "potential" $E$ while (4.7) and (4.9) describe properties of the heat kernel coefficients under local scale transformations.

To calculate the heat kernel coefficients we adopt the following strategy. First we write down a general expression for $a_{k}$ containing all invariants $\mathcal{A}_{n}^{I}$ of dimension $k$ with arbitrary coefficients $u^{I}$. The constants $u^{I}$ are then calculated by using the properties derived above. The first three coefficients read [244, 245]:

$$
\begin{aligned}
a_{0}(f, D) & =(4 \pi)^{-n / 2} \int_{M} d^{n} x \sqrt{g} \operatorname{tr}_{V}\left\{\alpha_{0} f\right\} \\
a_{2}(f, D) & =(4 \pi)^{-n / 2} \frac{1}{6} \int_{M} d^{n} x \sqrt{g} \operatorname{tr}_{V}\left\{f\left(\alpha_{1} E+\alpha_{2} R\right)\right\} \\
a_{4}(f, D) & =(4 \pi)^{-n / 2} \frac{1}{360} \int_{M} d^{n} x \sqrt{g} \operatorname{tr}_{V}\left\{f \left(\alpha_{3} E_{; k k}+\alpha_{4} R E+\alpha_{5} E^{2}\right.\right. \\
& \left.\left.+\alpha_{6} R_{; k k}+\alpha_{7} R^{2}+\alpha_{8} R_{i j} R_{i j}+\alpha_{9} R_{i j k l} R_{i j k l}+\alpha_{10} \Omega_{i j} \Omega_{i j}\right)\right\}
\end{aligned}
$$

Instead of the $u^{I}$ we use rescaled constants $\alpha_{I}$. By (4.6) the coefficients $\alpha_{I}$ are true constants, i.e. they do not depend on $n$. One can check that indeed 
no more invariants exist. For example, $R_{i j ; i j}$ is proportional to $R_{; j j}$ due to the Bianchi identity.

The coefficient $\alpha_{0}$ follows immediately from the heat kernel expansion for the "free" scalar Laplacian on $S^{1}$ (see eq. (4.4)). We obtain $\alpha_{0}=1$. Let us now use (4.8). First take $k=2$. Then

$$
\frac{1}{6} \int_{M} d^{n} x \sqrt{g} \operatorname{tr}_{V}\left\{\alpha_{1} F\right\}=\int_{M} d^{n} x \sqrt{g} \operatorname{tr}_{V}\{F\}
$$

This gives $\alpha_{1}=6$. Take $k=4$ to see

$$
\frac{1}{360} \int_{M} d^{n} x \sqrt{g} \operatorname{tr}_{V}\left\{\alpha_{4} F R+2 \alpha_{5} F E\right\}=\frac{1}{6} \int_{M} d^{n} x \sqrt{g} \operatorname{tr}_{V}\left\{\alpha_{1} F E+\alpha_{2} F R\right\}
$$

The equation (4.17) shows that $\alpha_{5}=180, \alpha_{4}=60 \alpha_{2}$.

To proceed further we need local scale transformations defined in (4.7) and (4.9). These scale transformations look similar to the local Weyl transformations but are not exactly the same. The scale transformations in (4.7) are designed in such a way that the operator $D$ always transforms covariantly. This is not the case of the Weyl transformations of an arbitrary operator of Laplace type. For example, the scalar Laplacian (3.4) is conformally covariant for a special value (3.5) of the conformal coupling only. Therefore, some of the basic quantities are transformed in a somewhat unusual way. The metrics transforms as $g_{\mu \nu} \rightarrow e^{2 \epsilon f} g_{\mu \nu}$ thus defining standard conformal properties of the Riemann tensor, the Ricci tensor and the scalar curvature. The functions $a^{\sigma}$ and $b$ in eq. (2.1) transform homogeneously. Transformation properties of $\omega_{\mu}$ and $E$ are then defined through (2.3) and (2.4). One can obtain the following relations $[244,245]$

$$
\begin{aligned}
& \left.\frac{d}{d \epsilon}\right|_{\epsilon=0} \sqrt{g}=n f \sqrt{g}, \\
& \left.\frac{d}{d \epsilon}\right|_{\epsilon=0} R_{i j k l}=-2 f R_{i j k l}+\delta_{j l} f_{; i k}+\delta_{; i k} f_{; j l}-\delta_{i l} f_{; j k}-\delta_{j k} f_{; i l}, \\
& \left.\frac{d}{d \epsilon}\right|_{\epsilon=0} E=-2 f E+\frac{1}{2}(n-2) f_{; i i}, \\
& \left.\frac{d}{d \epsilon}\right|_{\epsilon=0} R=-2 f R-2(n-1) f_{; i i}, \\
& \left.\frac{d}{d \epsilon}\right|_{\epsilon=0} E_{; k k}=-4 f E_{; k k}-2 f_{; k k} E+\frac{1}{2}(n-2) f_{; i i j j}+(n-6) f_{; k} E_{; k}, \\
& \left.\frac{d}{d \epsilon}\right|_{\epsilon=0} R E=-4 f R E+\frac{1}{2}(n-2) f_{; i i} R-2(n-1) f_{; i i} E, \\
& \left.\frac{d}{d \epsilon}\right|_{\epsilon=0} E^{2}=-4 f E^{2}+(n-2) f_{; i i} E,
\end{aligned}
$$




$$
\begin{aligned}
& \left.\frac{d}{d \epsilon}\right|_{\epsilon=0} R_{; k k}=-4 f R_{; k k}-2 f_{; k k} R-2(n-1) f_{; i i j j}+(n-6) f_{; i} R_{; i}, \\
& \left.\frac{d}{d \epsilon}\right|_{\epsilon=0} R^{2}=-4 f R^{2}-4(n-1) f_{; i i} R, \\
& \left.\frac{d}{d \epsilon}\right|_{\epsilon=0} R_{i j} R_{i j}=-4 f R_{i j} R_{i j}-2 f_{; i i} R-2(n-2) f_{; i j} R_{i j}, \\
& \left.\frac{d}{d \epsilon}\right|_{\epsilon=0} R_{i j k l} R_{i j k l}=-4 f R_{i j k l} R_{i j k l}-8 f_{; i j} R_{i j}, \\
& \left.\frac{d}{d \epsilon}\right|_{\epsilon=0} \Omega^{2}=-4 f \Omega^{2}
\end{aligned}
$$

Let us remind that the indices $i, j, k, l$ are flat, so we can put them all down and sum up over the repeated indices by contracting them with the Kronecker $\delta$.

Let us apply (4.9) to $n=4$.

$$
\left.\frac{d}{d \epsilon}\right|_{\epsilon=0} a_{2}\left(e^{-2 \epsilon f} F, e^{-2 \epsilon f} D\right)=0 .
$$

By collecting the terms with $\operatorname{tr}_{V} \int_{M} d^{n} x \sqrt{g}\left\{F f_{; j j}\right\}$ we obtain $\alpha_{1}=6 \alpha_{2}$ and consequently $\alpha_{2}=1$ and $\alpha_{4}=60 \alpha_{2}=60$. This completes calculation of $a_{2}$.

Let $M=M_{1} \times M_{2}$ with a product metric and let $D=\left(-\Delta_{1}\right)+\left(-\Delta_{2}\right)$ where $\Delta_{1,2}$ are scalar Laplacians on $M_{1}$ and $M_{2}$ respectively ${ }^{12}$. Eq. (4.2) yields

$$
\begin{gathered}
a_{4}\left(1,-\Delta_{1}-\Delta_{2}\right)=a_{4}\left(1,-\Delta_{1}\right) a_{0}\left(1,-\Delta_{2}\right)+a_{2}\left(1,-\Delta_{1}\right) a_{2}\left(1,-\Delta_{2}\right)+ \\
+a_{0}\left(1,-\Delta_{1}\right) a_{4}\left(1,-\Delta_{2}\right)
\end{gathered}
$$

It is clear from (3.6) that $E=0$ and $\Omega=0$. By collecting the terms with $R_{1} R_{2}$ (where $R_{1}$ and $R_{2}$ are scalar curvatures on $M_{1}$ and $M_{2}$ respectively) we obtain

Consequently, $\alpha_{7}=5$.

$$
\frac{2}{360} \alpha_{7}=\left(\frac{\alpha_{2}}{6}\right)^{2}
$$

Let us apply (4.9) to $n=6$. We obtain with the help of the variational equations (4.18)

$$
\begin{aligned}
0= & \operatorname{tr}_{V} \int_{M} d^{n} x \sqrt{g}\left\{F \left(\left(-2 \alpha_{3}-10 \alpha_{4}+4 \alpha_{5}\right) f_{; k k} E\right.\right. \\
& +\left(2 \alpha_{3}-10 \alpha_{6}\right) f_{; i i j j} \\
& +\left(2 \alpha_{4}-2 \alpha_{6}-20 \alpha_{7}-2 \alpha_{8}\right) f_{; i i} R \\
& \left.\left.+\left(-8 \alpha_{8}-8 \alpha_{9}\right) f_{; i j} R_{i j}\right)\right\} .
\end{aligned}
$$

The coefficients in front of independent invariants in (4.20) must be zero. We determine $\alpha_{3}=60, \alpha_{6}=12, \alpha_{8}=-2$ and $\alpha_{9}=2$.

\footnotetext{
$\overline{12}$ More precisely, we assume that in (3.4) the potential $U=0$, the conformal coupling is minimal $\xi=0$, and there is no gauge coupling $G_{\mu}=0$ on both $M_{1}$ and $M_{2}$.
} 
The most elegant way to calculate the remaining constant $\alpha_{10}$ is based on the Gauss-Bonnet theorem (see [245]). We use here a more lengthy way [337] which however works perfectly on flat manifolds without boundary and of trivial topology of $\mathbb{R}^{n}$. Note that $\mathbb{R}^{n}$ is non-compact. To make the heat kernel well defined we should suppose certain fall-off conditions on the background fields and on the smearing function $f$. The basis in the space of the square integrable functions is given by the plane waves $\exp (i k x)$. Therefore, for $M=$ $\mathbb{R}^{n}$ with flat metric the heat kernel reads:

$$
\begin{aligned}
& K(f ; t)=\operatorname{Tr}_{L^{2}}(f \exp (-t D)) \\
& \quad=\int d^{n} x \int \frac{d^{n} k}{(2 \pi)^{n}} e^{-i k x} \operatorname{tr}_{V}\left\{f(x) \exp (-t D) e^{i k x}\right\} \\
& \quad=\int d^{n} x \int \frac{d^{n} k}{(2 \pi)^{n}} \operatorname{tr}_{V}\left\{f(x) \exp \left(t\left(\left(\nabla^{\mu}+i k^{\mu}\right)^{2}+E\right)\right)\right\} .
\end{aligned}
$$

The following integrals will be useful:

$$
\begin{aligned}
& \int \frac{d^{n} k}{(2 \pi)^{n}} e^{-t k^{2}}=\frac{1}{(4 t \pi)^{n / 2}}, \\
& \int \frac{d^{n} k}{(2 \pi)^{n}} e^{-t k^{2}} k^{\mu} k^{\nu}=\frac{1}{(4 t \pi)^{n / 2}} \frac{1}{2 t} g^{\mu \nu} \\
& \int \frac{d^{n} k}{(2 \pi)^{n}} e^{-t k^{2}} k^{\mu} k^{\nu} k^{\rho} k^{\sigma}=\frac{1}{(4 t \pi)^{n / 2}} \frac{1}{4 t^{2}}\left(g^{\mu \nu} g^{\rho \sigma}+g^{\mu \rho} g^{\nu \sigma}+g^{\mu \sigma} g^{\nu \rho}\right) .
\end{aligned}
$$

Now we isolate $\exp \left(-t k^{2}\right)$ on the right hand side of (4.21) and expand the rest of the exponent in a power series of $t$.

$$
\begin{aligned}
K(f ; t) & =\int d^{n} x \int \frac{d^{4} k}{(2 \pi)^{n}} e^{-t k^{2}} \operatorname{tr}_{V}\left\{f ( x ) \left(1+t\left(\nabla^{2}+E\right)-\frac{t^{2}}{2} 4(k \nabla)\right.\right. \\
& +\frac{t^{2}}{2}\left(\nabla^{2} \nabla^{2}+\nabla^{2} E+E \nabla^{2}+E^{2}\right) \\
& -\frac{4 t^{3}}{6}\left((k \nabla)^{2} E+E(k \nabla)^{2}+(k \nabla) E(k \nabla)\right) \\
& -\frac{4 t^{3}}{6}\left((k \nabla)^{2} \nabla^{2}+\nabla^{2}(k \nabla)^{2}+(k \nabla) \nabla^{2}(k \nabla)\right) \\
& \left.\left.+\frac{16 t^{4}}{24}(k \nabla)^{4}+\ldots\right)\right\}
\end{aligned}
$$

We use the integrals (4.22) to obtain 


$$
\begin{aligned}
K(f ; t) & =\frac{1}{(4 \pi t)^{n / 2}} \int d^{n} x \operatorname{tr}_{V}\{f(x)(1+t E \\
& +\frac{t^{2}}{2}\left(\nabla^{2} \nabla^{2}+\nabla^{2} E+E \nabla^{2}+E^{2}\right) \\
& -\frac{t^{2}}{3}\left(\nabla^{2} E+E \nabla^{2}+\nabla^{\mu} E \nabla_{\mu}\right) \\
& -\frac{t^{2}}{3}\left(2 \nabla^{2} \nabla^{2}+\nabla^{\mu} \nabla^{2} \nabla_{\mu}\right) \\
& \left.\left.+\frac{t^{2}}{6}\left(\nabla^{\mu} \nabla^{\nu} \nabla_{\mu} \nabla_{\nu}+\nabla^{2} \nabla^{2}+\nabla^{\mu} \nabla^{2} \nabla_{\mu}\right)+O\left(t^{3}\right)\right)\right\} .
\end{aligned}
$$

All derivatives combine into commutators. Finally we get:

$$
\begin{aligned}
K(f ; t)=\frac{1}{(4 \pi t)^{n / 2}} \int d^{n} x \operatorname{tr}_{V}\{f(x)(1+t E \\
\left.\left.\quad+t^{2}\left(\frac{1}{2} E^{2}+\frac{1}{6} E_{; \mu \mu}+\frac{1}{12} \Omega_{\mu \nu} \Omega^{\mu \nu}\right)+O\left(t^{3}\right)\right)\right\} .
\end{aligned}
$$

From the equation (4.25) we conclude that $\alpha_{10}=30$. We have obtained also an independent confirmation for the values of $\alpha_{0}, \alpha_{1}, \alpha_{3}$ and $\alpha_{5}$.

The method we used above in eqs. (4.21) - (4.25) can be applied to more general manifolds and operators as well. The key ingredient is a convenient basis which should be used instead of the plane waves. For the case of a box with (anti-) periodic boundary condition such a basis is rather obvious (see $[13,14]$ ). On curved manifold $M$ one has to use the so-called geodesic waves $[220,221,425,427,347]$ although calculations with ordinary plane waves are also possible [120].

For calculation of the coefficient $a_{6}$ we refer to [244]. The results for the leading heat kernel coefficients are summarised in the following equations 


$$
\begin{aligned}
a_{0}(f, D)= & (4 \pi)^{-n / 2} \int_{M} d^{n} x \sqrt{g} \operatorname{tr}_{V}\{f\} . \\
a_{2}(f, D)= & (4 \pi)^{-n / 2} 6^{-1} \int_{M} d^{n} x \sqrt{g} \operatorname{tr}_{V}\{f(6 E+R)\} \\
a_{4}(f, D)= & (4 \pi)^{-n / 2} 360^{-1} \int_{M} d^{n} x \sqrt{g} \operatorname{tr}_{V}\left\{f \left(60 E_{; k k}+60 R E+180 E^{2}\right.\right. \\
& \left.\left.+12 R_{; k k}+5 R^{2}-2 R_{i j} R_{i j}+2 R_{i j k l} R_{i j k l}+30 \Omega_{i j} \Omega_{i j}\right)\right\} . \\
a_{6}(f, D)= & (4 \pi)^{-n / 2} \int_{M} d^{n} x \sqrt{g} \operatorname{tr}_{V}\left\{\frac { f } { 7 ! } \left(18 R_{; i j j}+17 R_{; k} R_{; k}-2 R_{i j ; k} R_{i j ; k}\right.\right. \\
& -4 R_{j k ; n} R_{j n ; k}+9 R_{i j k l ; n} R_{i j k l ; n}+28 R R_{; n n}-8 R_{j k} R_{j k ; n n} \\
& +24 R_{j k} R_{j n ; k n}+12 R_{i j k l} R_{i j k l ; n n}+35 / 9 R^{3}-14 / 3 R R_{i j} R_{i j} \\
& +14 / 3 R R_{i j k l} R_{i j k l}-208 / 9 R_{j k} R_{j n} R_{k n}-64 / 3 R_{i j} R_{k l} R_{i k j l} \\
& -16 / 3 R_{j k} R_{j n l i} R_{k n l i}-44 / 9 R_{i j k n} R_{i j l p} R_{k n l p} \\
& \left.-80 / 9 R_{i j k n} R_{i l k p} R_{j l n p}\right)+360^{-1} f\left(8 \Omega_{i j ; k} \Omega_{i j ; k}+2 \Omega_{i j ; j} \Omega_{i k ; k}\right. \\
& +12 \Omega_{i j ; k k} \Omega_{i j}-12 \Omega_{i j} \Omega_{j k} \Omega_{k i}-6 R_{i j k n} \Omega_{i j} \Omega_{k n}-4 R_{j k} \Omega_{j n} \Omega_{k n} \\
& +5 R \Omega_{k n} \Omega_{k n}+6 E_{; i i j j}+60 E E_{; i i}+30 E_{; i} E_{; i}+60 E^{3} \\
& +30 E \Omega_{i j} \Omega_{i j}+10 R E_{; k k}+4 R_{j k} E_{; j k}+12 R_{; k} E_{; k}+30 E E R \\
& \left.\left.+12 E R_{; k k}+5 E R^{2}-2 E R_{i j} R_{i j}+2 E R_{i j k l} R_{i j k l}\right)\right\} .
\end{aligned}
$$

Everyone who ever attempted calculations of the heat kernel coefficients on curved background for arbitrary spin should appreciate that the method presented here is a quite efficient one. One should also take into account that some of the universal constant were indeed calculated twice.

The coefficients $a_{0}-a_{4}$ are contained in $[147,319] . a_{6}$ was first computed by Gilkey [244]. The next coefficient $a_{8}$ has been calculated by Amsterdamski et al [12] for the scalar Laplacian and by Avramidi [25, 26] for the general operator of Laplace type. The coefficient $a_{10}$ has been calculated by van de Ven [399]. Higher heat kernel coefficients in flat space were studied in $[205,206]$.

\subsection{Examples}

Here we consider several simple physical systems in four dimensions and calculate the heat kernel coefficient $a_{4}$ which defines the one-loop divergences in the zeta function regularization.

\subsubsection{Yang-Mills theory in flat space}

Our first example is pure Yang-Mills theory in flat space. We are interested in the "total" heat kernel coefficient $a_{4}^{\text {tot }}$ defined by (3.53). Let us start with the first term describing contribution from the vector fields. We choose the gauge (3.44). The only non-vanishing invariants are $E$ and $\Omega$. The coefficient $a_{4}$ is quadratic in these quantities. By using eqs. (3.46) and (3.47) we obtain: 


$$
\begin{aligned}
& \operatorname{tr}_{V}\left(E^{2}\right)=E_{\nu \beta}^{\alpha \rho} E_{\rho \alpha}^{\beta \nu}=4 F_{\rho \nu}^{\delta} F_{\rho \nu}^{\gamma} K_{\delta \gamma} \\
& \operatorname{tr}_{V}\left(\Omega_{i j} \Omega_{i j}\right)=-4 F_{\rho \nu}^{\delta} F_{\rho \nu}^{\gamma} K_{\delta \gamma}
\end{aligned}
$$

where

$$
K_{\delta \gamma}=c_{\alpha \beta}^{\delta} c_{\alpha \beta}^{\gamma}
$$

is the Killing form of the gauge algebra.

Let us remind that the indices $i, j, k$ in (4.28) refer to a local orthonormal frame. In flat space they may be identified with the vector indices $\mu, \nu, \rho$. In the case of vector fields the trace in (4.30) and (4.31) is taken over pairs consisting of a gauge index $\alpha, \beta$ or $\gamma$ and of a vector index $\mu, \nu$ or $\rho$.

For the ghost operator we have to put $R=U=0$ in (3.6) and identify the connection with the background gauge field according to (3.49). Consequently, $E^{[\mathrm{gh}]}=0$ and

$$
\operatorname{tr}_{V}\left(\Omega_{i j}^{[\mathrm{gh}]} \Omega_{i j}^{[\mathrm{gh}]}\right)=-F_{\rho \nu}^{\delta} F_{\rho \nu}^{\gamma} K_{\delta \gamma} .
$$

Next we substitute (4.30), (4.31) and (4.33) in (4.28) to obtain

$$
a_{4}^{[\mathrm{tot}]}=a_{4}^{[\mathrm{vec}]}-2 a_{4}^{[\mathrm{gh}]}=\frac{11}{96 \pi^{2}} \int_{M} d^{4} x \sqrt{g} F_{\rho \nu}^{\delta} F_{\rho \nu}^{\gamma} K_{\delta \gamma}
$$

The Yang-Mills gauge group $\mathcal{G}$ has usually a direct product structure, $\mathcal{G}=\mathcal{G}_{1} \times \mathcal{G}_{2} \times \ldots$, so that on each of the irreducible components $\mathcal{G}_{i}$ the Killing form $K$ is proportional to the unit matrix. Therefore, the one-loop divergence (4.34) reproduces the structure of the classical action with different charges for each $\mathcal{G}_{i}$. We also recover the coefficient $11 / 3$ which is familiar from computations of the Yang-Mills beta functions.

\subsubsection{Free fields in curved space}

Consider free quantum fields on a Riemannian manifold without boundaries. "Free" means that we neglect all interactions except for the one with the background geometry. The heat kernel coefficients can be expressed then in terms of local invariants of the metric. In particular,

$$
a_{4}(x)=\frac{1}{2880 \pi^{2}}\left[a C_{\mu \nu \rho \sigma} C^{\mu \nu \rho \sigma}+b\left(R_{\mu \nu} R^{\mu \nu}-\frac{1}{3} R^{2}\right)+c R_{; \mu}{ }^{\mu}+d R^{2}\right],
$$

where $C_{\mu \nu \rho \sigma}$ is the Weyl tensor,

$$
C_{\mu \nu \rho \sigma} C^{\mu \nu \rho \sigma}=R_{\mu \nu \rho \sigma} R^{\mu \nu \rho \sigma}-2 R_{\mu \nu} R^{\mu \nu}+\frac{1}{3} R^{2}
$$

$a, b, c$ and $d$ are some constants depending on the spin. The first two structures in (4.35) which appear together with $a$ and $b$ are conformally invariant in four dimensions. This explains our choice of the basis in the space of invariants.

The constants $a, b, c$ and $d$ can be evaluated by substituting particular expressions for $E$ and $\Omega$ obtained in sec. 3 in (4.28). Alternatively, one can use the analysis of Christensen and Duff [127] who calculated $a_{4}$ for arbitrary spin 
Table 1

$a_{4}$ for various spins

\begin{tabular}{|l|c|c|c|c|}
\hline Spin & $a$ & $b$ & $c$ & $d$ \\
\hline 0 & 1 & 1 & $30 \xi-6$ & $90(\xi-1 / 6)^{2}$ \\
$1 / 2$ & $-7 / 2$ & -11 & 6 & 0 \\
1 & -13 & 62 & 18 & 0 \\
2 & 212 & 0 & 0 & $717 / 4$ \\
\hline
\end{tabular}

fields (see also [69]). The results are collected in Table 1. Some comments are in order. Spin 1/2 means 4-component Dirac spinors. For spin 1 and spin 2 fields we took into account contributions from corresponding ghost fields. Note, that vector Yang-Mills fields and vector ghosts for gravity interact differently with the background geometry. The cosmological constant is taken to be zero.

As a physical application of the heat kernel expansion in curved space we may mention, for example, the asymptotic conformal invariance phenomenon which was studied by using this technique in [103, 104, 99, 108].

A similar analysis can be performed also in the presence of boundaries [333].

\subsection{DeWitt iterative procedure}

The iterative method by DeWitt uses separation of the heat kernel with non-coinciding arguments into a leading part (which is non-analytic in $t$ ) and the power-law corrections. For a flat manifold this separation has been described in sec. 1 (cf. eqs. (1.12) and (1.13)). Let us consider a massless scalar field on a curved compact Riemannian manifold $M$. The DeWitt ansatz reads in this case:

$$
K(t ; x, y ; D)=(4 \pi t)^{-n / 2} \Delta_{V V M}^{1 / 2}(x, y) \exp \left(-\frac{\sigma(x, y)}{2 t}\right) \Xi(t ; x, y ; D)
$$

where $\sigma(x, y)$ is one half the square of the length of the geodesic connecting $x$ and $y$. In Cartesian coordinates on a flat manifold $\sigma_{\text {flat }}(x, y)=\frac{1}{2}(x-y)^{2}$. $\Delta_{V V M}$ is the so called Van Vleck-Morette determinant

$$
\Delta_{V V M}(x, y)=\frac{\operatorname{det}\left(-\frac{\partial}{\partial x^{\mu}} \frac{\partial}{\partial y^{\nu}} \sigma(x, y)\right)}{\sqrt{g(x) g(y)}} .
$$

As a consequence of the heat equation (1.10) the kernel $\Xi$ should satisfy

$$
\left(\partial_{t}+t^{-1}\left(\nabla^{\mu} \sigma\right) \nabla_{\mu}+\Delta_{V V M}^{-1 / 2} D \Delta_{V V M}^{1 / 2}\right) \Xi=0
$$


with the initial condition

$$
\Xi(0 ; x, y ; D)=1
$$

The essence of the DeWitt method is to look for a solution of the equation (4.39) in the following form

$$
\Xi(t ; x, y ; D)=\sum_{j=0}^{\infty} t^{j} b_{2 j}(x, y ; D) .
$$

The initial condition (4.40) yields

$$
b_{0}=1
$$

The recursion relation

$$
\left(j+\left(\nabla^{\mu} \sigma\right) \nabla_{\mu}\right) b_{2 j}+\Delta_{V V M}^{-1 / 2} D \Delta_{V V M}^{1 / 2} b_{2(j-1)}=0,
$$

which follows from (4.39) and (4.41), allows, in principle, to find the coincidence limits $x=y$ of higher heat kernel coefficients $b_{2 j}$. An important ingredient of such calculations is the coincidence limits of symmetrized derivatives of the geodesic interval $\sigma(x, y)[395,55]$. This method becomes very cumbersome beyond $a_{4}$. A refined nonrecursive procedure to solve the DeWitt equation (4.43) was used by Avramidi [26] to calculate $a_{8}$ (see also [34] for a short overview). The method of DeWitt can be naturally extended to treat coincidence limits of the derivatives of $b_{2 j}(x, y ; D)$.

The recursion relations can be generalised to the case of manifolds with boundaries $[315,318]$. However, for a practical use the functorial methods of sec. 5 seem to be more convenient.

\section{$4.4 \quad$ Non-minimal operators}

Quantisation of gauge theories oftenly leads to second order differential operators which are not of the Laplace type. For example, by taking $\kappa \neq 1$ in (3.52) one obtains the following operator acting on the gauge field fluctuations:

$$
D_{\mu \nu}^{[\mathrm{nm}]}=-\Delta g_{\mu \nu}+\left(1-\frac{1}{\kappa}\right) \nabla_{\nu} \nabla_{\mu},
$$

where, for simplicity, we suppose that the manifold $M$ is flat $\left(R_{\mu \nu \rho \sigma}=0\right)$ and the gauge group is abelian $\left(c_{\alpha \beta}^{\gamma}=0\right)$. The leading symbol of the operator (4.44) (the part with the highest derivatives) has a non-trivial matrix structure. Such operators are called non-minimal.

In some simple cases (see, e.g., $[240,7,411])$ the spectral problem for nonminimal operators can be reduced to the Laplacians. If the leading part of a non-minimal operator has a form similar to (4.44) but the lower order part is more or less arbitrary ${ }^{13}$, necessary generalisations of the DeWitt technique

\footnotetext{
$\overline{13}$ This case covers most of the physical applications.
} 
were suggested by Barvinsky and Vilkovisky [55]. The technique was further developed in $[269,123]$. Complete calculation of $a_{4}$ required extensive use of the computer algebra [270]. Most general non-minimal operators were considered in [32] where, because of great technical complexity, only the first two heat kernel coefficients were analysed. Some calculations in various physical systems with non-minimal operators can be found in [209,357,266,361]. 


\section{Heat kernel expansion on manifolds with boundaries}

\subsection{Two particular cases}

We start our analysis of manifolds with boundaries with two simple examples. First, let us consider a one-dimensional manifold $M=[0, \pi]$. Let $D=-\partial_{x}^{2}$. We consider both Dirichlet (2.15) and Neumann (2.16) boundary conditions (taking $S=0$ in (2.16) to simplify the calculations). The eigenfunctions of $D$ are:

Dirichlet: $\sin (l x), l=1,2, \ldots$;

Neumann: $\cos (l x), l=0,1,2, \ldots$

The eigenvalues are $l^{2}$ in both cases, where $l$ is a positive integer for Dirichlet boundary conditions, and $l$ is a nonnegative integer for Neumann boundary conditions. The heat kernel asymptotics can be calculated with the help of the Poisson summation formula (4.4):

$$
\begin{aligned}
& K\left(t,-\partial_{x}^{2}, \mathcal{B}^{-}\right)=\sum_{l>0} \exp \left(-t l^{2}\right)=\frac{1}{2}\left(\sqrt{\frac{\pi}{t}}-1\right)+\mathcal{O}\left(e^{-1 / t}\right) \\
& K\left(t,-\partial_{x}^{2}, \mathcal{B}^{+}\right)=\sum_{l \geq 0} \exp \left(-t l^{2}\right)=\frac{1}{2}\left(\sqrt{\frac{\pi}{t}}+1\right)+\mathcal{O}\left(e^{-1 / t}\right)
\end{aligned}
$$

where for the later use we explicitly mention the boundary operators $\mathcal{B}^{-}$and $\mathcal{B}^{+}$which define Dirichlet (2.15) and Neumann (2.16) boundary conditions respectively.

Let us modify a little bit the example above by allowing for a non-zero $S$ at one of the components of the boundary:

$$
\left.\partial_{x} \phi\right|_{x=0}=0,\left.\quad\left(\partial_{x}-S\right) \phi\right|_{x=\pi}=0
$$

Note, that at $x=\pi$ the derivative with respect to an inward pointing unit normal is $-\partial_{x}$. The eigenfunctions

$$
\phi_{k}=\cos (k x)
$$

satisfy the boundary conditions (5.4) at $x=0$. The spectrum is defined by the condition at $x=\pi$ which reads ${ }^{14}$ :

$$
-k \sin (k \pi)=S \cos (k \pi) .
$$

In this example we restrict ourselves to the linear order in $S$. We suppose that $S$ is small and that the spectrum (5.1) is only slightly perturbed:

$$
k_{l}=l+z_{l} .
$$

\footnotetext{
$\overline{{ }^{14} \text { For } S}>0$ also a negative mode $\cosh (k x)$ appears. Here we take $S \leq 0$.
} 
The equation (5.6) gives in this approximation

$$
\begin{array}{lll}
z_{l}=-\frac{S}{l \pi} & \text { for } & l>0, \\
z_{0}^{2}=-\frac{S}{\pi} & \text { for } & l=0 .
\end{array}
$$

We expand also the heat kernel in a power series in $S$ and use (5.8) and (5.2) to obtain:

$$
\begin{aligned}
K\left(t,-\partial_{x}^{2}, \mathcal{B}_{S}^{+}\right) & =\sum_{l \geq 0} \exp \left(-t k_{l}^{2}\right) \\
& =\sum_{l>0} \exp \left(-t l^{2}\right)\left(1+\frac{2 S t}{\pi}\right)+1+\frac{S t}{\pi}+\mathcal{O}\left(S^{2}\right) \\
& =\frac{1}{2}\left(\sqrt{\frac{\pi}{t}}+1\right)+S \sqrt{\frac{t}{\pi}}+\mathcal{O}\left(S^{2}\right),
\end{aligned}
$$

where we also dropped exponentially small terms in $t$.

Our next example is the heat kernel expansion for scalar fields in a ball with Dirichlet and Neumann boundary conditions [392,416, 291,328,72]. The metric of the unit ball in $\mathbb{R}^{n}$ reads:

$$
d s^{2}=d r^{2}+r^{2} d \Omega^{2}, \quad 0 \leq r \leq 1
$$

where $d \Omega^{2}$ is the metric on unit sphere $S^{n-1}$. The scalar Laplace operator has the form

$$
\Delta \phi=\left(\partial_{r}^{2}+\frac{n-1}{r} \partial_{r}+{ }^{(n-1)} \Delta\right) \phi,
$$

where $^{(n-1)} \Delta$ is the Laplace operator on $S^{n-1}$. The eigenfunctions of the operator (5.11) are well known

$$
\phi_{l, \lambda} \propto r^{(2-n) / 2} J_{(n-2) / 2+l}\left(r \lambda^{1 / 2}\right) Y_{(l)}\left(x^{a}\right) .
$$

$J_{p}$ are the Bessel functions. The eigenvalues $-\lambda$ are defined by boundary conditions. $Y_{(l)}\left(x^{i}\right)$ are $n$-dimensional scalar spherical harmonics. Their degeneracies are

$$
N_{l}=\frac{(2 l+n-2)(l+n-3) !}{l !(n-2) !} .
$$

To proceed further it is convenient to consider the zeta function which may be presented through a contour integral $[72]^{15}$ :

$$
\zeta(s, D)=\sum_{l=0}^{\infty} N_{l} \int_{\gamma} \frac{d k}{2 \pi i} k^{-2 s} \frac{\partial}{\partial k} \Phi_{(n-2) / 2+l}(k),
$$

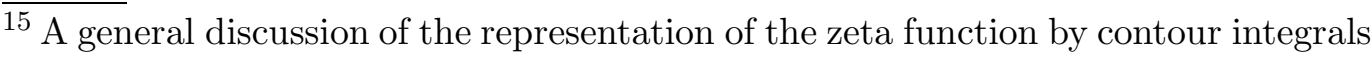
can be found in [299].
} 
where $\Phi_{\nu}$ is a function which has zeros at the spectrum $k=\sqrt{\lambda}$. For Dirichlet boundary conditions this function reads:

$$
\Phi_{\nu}=k^{-\nu} J_{\nu}(k)
$$

For Robin boundary condition $\Phi_{\nu}$ is given by a somewhat more complicated combination of the Bessel functions. The contour $\gamma$ runs counterclockwise and encloses all the solutions of $\Phi_{\nu}=0$ on the positive real axis. Note the presence of $k^{-\nu}$ in (5.15) which is included to avoid unwanted contributions coming from the origin $k=0$.

The next step is to rotate the contour to the imaginary axis and to calculate residues of $\Gamma(s) \zeta(s, D)$ (as prescribed by $(2.26)$ ). It is interesting to note that the heat kernel coefficients are defined by several leading terms in the uniform asymptotic expansion of $\Phi_{\nu}(i \nu z)$ for large $\nu$ and fixed $z$. For further details we refer to [72].

Euclidean ball was frequently used in calculations of the heat kernel coefficients and functional determinants. Apart from the papers already quoted above also the computations for a scalar field [163,75, 158,73,339], spinors $[141,288,289,159,164,298,179,199]$, abelian gauge fields [307, 196, 192, 404, $410,159,298,194,197,181,195,64]^{16}$ should be mentioned. A similar technique works also for more complicated geometries as, e.g., the spherical cap $[52,287,51,288,163]$. Note that here we consider local boundary conditions without tangential derivatives only. For other types of boundary operators see sec. 5.4 .

\subsection{Dirichlet and Neumann boundary conditions}

Let us now find analytic expressions for the heat kernel coefficients in terms of geometric invariants. Here we follow the method of Branson and Gilkey [90,245]. For both Dirichlet and modified Neumann (Robin) boundary conditions the heat kernel coefficients are locally computable. This means that $a_{k}$ may be represented as a sum of volume and boundary integrals of some local invariants:

$$
a_{k}(f, D, \mathcal{B})=\int_{M} d^{n} x \sqrt{g} f(x) a_{k}(x, D)+\sum_{j=0}^{k-1} \int_{\partial M} d^{n-1} x \sqrt{h} f^{(j)} a_{k, j}(x, D, \mathcal{B}),
$$

where $f^{(j)}$ denotes $j$-th normal derivative of the smearing function $f$. Note, that we cannot now integrate by parts to remove normal derivatives from $f$. This reflects the distributional nature of the heat kernel asymptotics.

\footnotetext{
${ }^{16}$ Some of these works consider contributions of the so called physical modes only. As explained in [413] complete answer for the effective action and for the scaling behaviour must include contributions from ghosts and non-physical modes. This applies also to the spherical cap case considered below.
} 
The volume terms $a_{k}(x, D)$ are the same as in the previous section (see $(4.26)$ - (4.29)). Here we evaluate the boundary terms $a_{k, j}(x, D, \mathcal{B})$. Obviously, the canonical mass dimension of $a_{k, j}$ is $k-j-1$. In addition to the usual bulk invariants $E, R$, etc, $a_{k, j}$ can also contain specific boundary quantities as the extrinsic curvature $L_{a b}$ or $S$ (for Robin boundary conditions). Note, that $L_{a b}$ and $S$ are defined on the boundary only and, therefore, can be differentiated only tangentially ${ }^{17}$. Canonical mass dimension of $L_{a b}$ and $S$ is +1 .

In this section we explicitly calculate the first three heat kernel coefficients $a_{0}, a_{1}$ and $a_{2}$. Basing on the considerations of the preceding paragraph we may write:

$$
\begin{aligned}
& a_{0}\left(f, D, \mathcal{B}^{ \pm}\right)=(4 \pi)^{-n / 2} \int_{M} d^{n} x \sqrt{g} \operatorname{tr}_{V}(f), \\
& a_{1}\left(f, D, \mathcal{B}^{ \pm}\right)=(4 \pi)^{-(n-1) / 2} \int_{\partial M} d^{n-1} x \sqrt{h} b_{1}^{ \pm} \operatorname{tr}_{V}(f), \\
& a_{2}\left(f, D, \mathcal{B}^{ \pm}\right)=\frac{1}{6}(4 \pi)^{-n / 2}\left\{\int_{M} d^{n} x \sqrt{g} \operatorname{tr}_{V}(6 f E+f R)\right. \\
&\left.+\int_{\partial M} d^{n-1} x \sqrt{h} \operatorname{tr}_{V}\left(b_{2}^{ \pm} f L_{a a}+b_{3}^{ \pm} f_{; n}+b_{4}^{ \pm} f S\right)\right\},
\end{aligned}
$$

where $\mathcal{B}^{ \pm}$denotes either Robin $\left(\mathcal{B}^{+}\right)$or Dirichlet $\left(\mathcal{B}^{-}\right)$boundary operator. $b^{ \pm}$ are some constants. To keep uniform notations we formally included $S$ also in the expression for Dirichlet boundary conditions. It should be assumed that $S=0$ for that case.

Since the product formula (4.2) is still valid with obvious modifications for the boundary contributions, we can again consider the case $M=M_{1} \times S^{1}$ and repeat step by step the calculations (4.3) - (4.6) thus arriving to the same conclusion that the constants $b^{ \pm}$do not depend on dimension of the manifold (all explicit $n$-dependence of the heat kernel is contained in the power of $4 \pi$ ). This property is crucial for our calculations.

The one dimensional examples ${ }^{18}$ (5.2) and (5.3) immediately give

$$
b_{1}^{+}=-b_{1}^{-}=\frac{1}{4} .
$$

Our next example (5.9) controls linear terms in $S$ and gives

$$
b_{4}^{+}=12 .
$$

For Dirichlet boundary conditions we have set $S=0$. Therefore, $b_{4}^{-}$plays no role.

To define the constants $b_{2}^{ \pm}$and $b_{3}^{ \pm}$we use the conformal variation equation (4.7). Variations of $E$ and $R$ are given by (4.18). Conformal properties of the

\footnotetext{
${ }^{17}$ See sec. 2.1 for more information on differential geometry of manifolds with boundary.

${ }^{18}$ For $M=[0, \pi]$ the boundary integral reduces to a sum of two contributions from $x=0$ and $x=\pi$.
} 
second fundamental form $L_{a b}$ are dictated by that of the metric:

$$
\left.\frac{d}{d \epsilon}\right|_{\epsilon=0} L_{a a}=-f L_{a a}-(n-1) f_{; n} .
$$

We wish to keep the boundary conditions invariant under the conformal transformations. This implies that the boundary operators $\mathcal{B}^{ \pm}$must transform homogeneously. This property holds automatically for Dirichlet boundary conditions (2.15). In the case of modified Neumann boundary conditions conformal transformation of $S$ should cancel inhomogeneous term in the connection $\omega_{n}$. This yields

$$
\left.\frac{d}{d \epsilon}\right|_{\epsilon=0} S=-f S+\frac{1}{2}(n-2) f_{; n} .
$$

Next we substitute the variational formulae (4.18), (5.22) and (5.23) in (4.7) with $k=2$. We collect the terms with $f_{; n}$ on the boundary to obtain

$$
-(n-4)-(n-1) b_{2}^{-}=(n-2) b_{3}^{-}
$$

for Dirichlet conditions and

$$
-(n-4)-(n-1) b_{2}^{+}+6(n-2)=(n-2) b_{3}^{+}
$$

for generalised Neumann (Robin) ones. Since the constants $b^{ \pm}$do not depend on $n$ the two equations (5.24) and (5.25) are enough to define these constants:

$$
b_{2}^{+}=b_{2}^{-}=2, \quad b_{3}^{+}=-b_{3}^{-}=3 .
$$

This completes the calculation of $a_{2}$.

The coefficients $a_{3}$ and $a_{4}$ can be obtained as particular cases of more general formulae of the next subsection.

\subsection{Mixed boundary conditions}

Let us now turn to mixed boundary conditions (2.17) which, as we have seen in sections 3.3 and 3.4, are natural boundary conditions for spinor and vector fields. These boundary conditions depend on two complementary local projectors $\Pi_{-}$and $\Pi_{+}=1-\Pi_{-}$which define subsets of components of the field satisfying Dirichlet and Robin boundary conditions respectively ${ }^{19}$. More precisely,

$$
\left.\Pi_{-} \phi\right|_{\partial M}=0,\left.\quad\left(\nabla_{n}+S\right) \Pi_{+} \phi\right|_{\partial M}=0 .
$$

Consequently, there is one more independent entity (as compared to Robin case, for example) on which the heat kernel coefficients for mixed boundary conditions can depend. This makes the calculations somewhat more complicated. It is convenient to define

$$
\chi=\Pi_{+}-\Pi_{-} .
$$

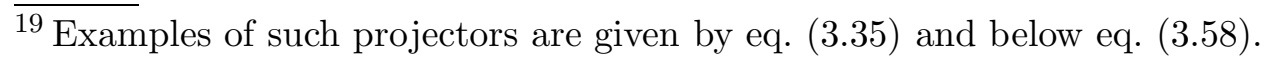


Calculation of the coefficients $a_{k}$ up to $k=4$ can be found in [90] (see also [404] for some corrections). The result reads:

$$
\begin{aligned}
& a_{0}(f, D, \mathcal{B})=(4 \pi)^{-n / 2} \int_{M} d^{n} x \sqrt{g} \operatorname{tr}_{V}(f) \\
& a_{1}(f, D, \mathcal{B})= \frac{1}{4}(4 \pi)^{-(n-1) / 2} \int_{\partial M} d^{n-1} x \sqrt{h} \operatorname{tr}_{V}(\chi f) \\
& a_{2}(f, D, \mathcal{B})=\frac{1}{6}(4 \pi)^{-n / 2}\left\{\int_{M} d^{n} x \sqrt{g} \operatorname{tr}_{V}(6 f E+f R)\right. \\
&\left.+\int_{\partial M} d^{n-1} x \sqrt{h} \operatorname{tr}_{V}\left(2 f L_{a a}+3 \chi f_{; n}+12 f S\right)\right\} \\
& a_{3}(f, D, \mathcal{B})=\frac{1}{384}(4 \pi)^{-(n-1) / 2} \int_{\partial M} d^{n-1} x \sqrt{h} \operatorname{tr}_{V}\{f(96 \chi E+16 \chi R \\
&+8 f \chi R_{a n a n}+\left(13 \Pi_{+}-7 \Pi_{-}\right) L_{a a} L_{b b}+\left(2 \Pi_{+}+10 \Pi_{-}\right) L_{a b} L_{a b} \\
&\left.+96 S L_{a a}+192 S^{2}-12 \chi_{: a} \chi_{: a}\right)+f_{; n}\left(\left(6 \Pi_{+}+30 \Pi_{-}\right) L_{a a}\right. \\
&\left.+96 S)+24 \chi f_{; n n}\right\} \\
& a_{4}(f, D, \mathcal{B})=\frac{1}{360}(4 \pi)^{-n / 2}\left\{\int _ { M } d ^ { n } x \sqrt { g } _ { \operatorname { t r } _ { V } } \left\{f \left(60 E_{; i i}+60 R E+180 E^{2}\right.\right.\right. \\
&\left.\left.+30 \Omega_{i j} \Omega_{i j}+12 R_{; i i}+5 R^{2}-2 R_{i j} R_{i j}+2 R_{i j k l} R_{i j k l}\right)\right\} \\
&+\int_{\partial M} d^{n-1} x \sqrt{h} \operatorname{tr}_{V}\left\{f \left\{\left(240 \Pi_{+}-120 \Pi_{-}\right) E_{; n}\right.\right. \\
&+\left(42 \Pi_{+}-18 \Pi_{-}\right) R_{; n}+24 L_{a a: b b}+0 L_{a b: a b}+120 E L_{a a} \\
&+20 R L_{a a}+4 R_{a n a n} L_{b b}-12 R_{a n b n} L_{a b}+4 R_{a b c b} L_{a c} \\
&+\frac{1}{21}\left\{\left(280 \Pi_{+}+40 \Pi_{-}\right) L_{a a} L_{b b} L_{c c}+\left(168 \Pi_{+}\right.\right. \\
&\left.\left.+264 \Pi_{-}\right) L_{a b} L_{a b} L_{c c}+\left(224 \Pi_{+}+320 \Pi_{-}\right) L_{a b} L_{b c} L_{a c}\right\} \\
&+720 S E+120 S R+0 S R_{a n a n}+144 S L_{a a} L_{b b}+48 S L_{a b} L_{a b} \\
&+480 S^{2} L_{a a}+480 S^{3}+120 S_{: a a}+60 \chi \chi_{: a} \Omega_{a n}-12 \chi_{: a} \chi_{: a} L_{b b} \\
&\left.+24 \chi_{: a} \chi_{: b} L_{a b}-120 \chi_{: a} \chi_{: a} S\right\}+f_{; n}\left(180 \chi \chi_{+}+30 \chi R+0 R_{a n a n}\right. \\
&+\frac{1}{7}\left\{\left(84 \Pi_{+}-180 \Pi_{-}\right) L_{a a} L_{b b}+\left(84 \Pi_{+}+60 \Pi_{-}\right) L_{a b} L_{a b}\right\} \\
&\left.+72 S L_{a a}+240 S^{2}-18 \chi_{: a} \chi_{: a}\right)+f_{; n n}\left(24 L_{a a}+120 S\right) \\
&\left.\left.+30 \chi f_{; i i n}\right\}\right\} \\
&+
\end{aligned}
$$

Dirichlet and modified Neumann (Robin) boundary conditions are recovered when $\Pi_{+}=0$ or $\Pi_{-}=0$ respectively.

The coefficients $a_{k}$ with $k=0,1,2,3,4$ were calculated in [319, 292,388, $320,329,90,315,318,404]$. A different algorithm was suggested in [131]. For "pure" (not mixed) boundary conditions the coefficient $a_{5}$ was calculated by Branson, Gilkey and Vassilevich [93] for the special case of a domain in flat space or of a curved domain with totally geodesic boundaries. Kirsten [296] generalised these results for arbitrary manifolds and boundaries. Branson, Gilkey, Kirsten and Vassilevich [91] calculated the coefficient $a_{5}$ for mixed boundary conditions. 


\subsection{Other boundary conditions}

From the technical point of view boundary conditions for a Laplace type operator are needed to exclude infinite number of negative and zero modes and to ensure self-adjontness. In principle, any linear relation between the boundary data $\left.\phi\right|_{\partial M}$ and $\left.\phi_{; n}\right|_{\partial M}$ is admissible as long as it serves this purpose. Here we consider two physically motivated examples of boundary conditions which contain tangential derivatives on the boundary. These examples should give the reader an idea of what can be expected for a more general boundary value problem.

\subsubsection{Boundary conditions with tangential derivatives and Born-Infeld ac- tion from open strings}

The boundary condition

$$
\left.\left(\nabla_{n}+\frac{1}{2}\left(\nabla_{a} \Gamma_{a}+\Gamma_{a} \nabla_{a}\right)+S\right) \phi\right|_{\partial M}=0
$$

is the simplest condition containing both normal and tangential derivatives. $\Gamma_{a}$ and $S$ are some matrix valued functions defined on the boundary. Such or similar structures appear in open strings (cf. sec. 3.2) and in chiral bags (cf. sec. 3.3). They also describe photons with the Chern-Simons interaction term concentrated on the boundary $[184,83]$ and may be relevant for solid state physics applications. The conditions (5.34) appeared in the mathematical literature $[257,243]$.

Several heat kernel coefficients for the boundary conditions (5.34) (which are called oblique) have been calculated by McAvity and Osborn [316] and by Dowker and Kirsten [167,168]. Avramidi and Esposito [37,36,38,35] lifted some commutativity assumptions and proved a simple criterion of strong ellipticity (see below).

Consider a differential operator $Q$, not necessarily of the second order. Let is separate the part containing highest derivatives and replace $\partial_{\mu} \rightarrow i k_{\mu}$, like in doing the Fourier transformation. In this way we obtain an object $A_{Q}=$ $a^{\mu \nu \ldots \rho}(x) k_{\mu} k_{\nu} \ldots k_{\rho}$ which is called the leading symbol of $Q$. If the operator $Q$ acts on fields with indices (i.e. in a vector bundle), the index structure of $Q$ is inherited by $A_{Q}$. In other words, for fixed $k$ the leading symbol $A$ is a matrix valued function (an endomorphism of the vector bundle). If $A_{Q}(x)$ is non-degenerate for all $k \neq 0$, the operator $Q$ is called elliptic. For the

Dirac operator $Q=\not D$ the leading symbol is $A_{Q}=\gamma^{\mu} k_{\mu}$. Therefore, $\not D$ is obviously elliptic. The Laplace type operators are also elliptic since $A_{D}=k^{2}$. Ellipticity means that "at large momenta" the operator $Q$ is dominated by its' highest derivative part. This is the property which guarantees that on a compact manifold without boundaries Laplace operators have at most finite number of negative and zero eigenvalues and which ensures existence of the heat kernel. On manifolds with boundaries just ellipticity is not enough to 
ensure nice properties of the spectrum. There is an additional requirement, called strong ellipticity, which should be satisfied by the boundary operator (see [245] for details). Dirichlet and Robin boundary conditions are always strongly elliptic. Oblique boundary conditions are strongly elliptic if and only if $\left|\Gamma^{2}\right|<1$. If this inequality is violated, infinite number of negative eigenmodes appears (a simple example can be found in Appendix B of [306]). Note that the boundary conditions (5.34) correspond to a symmetric $D$ if the matrices $\Gamma_{a}$ are anti-hermitian. Therefore, $\Gamma^{2}=\Gamma_{a} \Gamma^{a}$ is typically negative.

Another complication stems from the fact that $\Gamma_{a}$ is dimensionless. Consequently, arbitrary powers of $\Gamma_{a}$ may enter $a_{k}$, so that instead of the undetermined constants (cf. (5.17) - (5.19)) one has to deal with undetermined functions of $\Gamma$. The problem becomes more tractable if we suppose that $\Gamma_{a}$ 's commute among themselves, $\left[\Gamma_{a}, \Gamma_{b}\right]=0$. For this case, the coefficients $a_{1}$ and $a_{2}$ have been calculated by McAvity and Osborn [316] $\left(a_{0}\right.$ is still given by $(5.17))$ :

$$
\begin{aligned}
a_{1}(f, D) & =(4 \pi)^{-(n-1) / 2} \int_{\partial M} d^{n-1} x \sqrt{h} \operatorname{tr}_{V}(f \gamma(\Gamma)) \\
a_{2}(f, D) & =\frac{1}{6}(4 \pi)^{-n / 2}\left\{\int_{M} d^{n} x \sqrt{g} \operatorname{tr}_{V}(6 f E+f R)\right. \\
& +\int_{\partial M} d^{n-1} x \sqrt{h} \operatorname{tr}_{V}\left(b_{0}(\Gamma) f L_{a a}+b_{1}(\Gamma) f_{; n}\right. \\
& \left.\left.+b_{2}(\Gamma) f S+f \sigma(\Gamma) L_{a b} \Gamma_{a} \Gamma_{b}\right)\right\}
\end{aligned}
$$

where

$$
\begin{aligned}
\gamma & =\frac{1}{4}\left[\frac{2}{\sqrt{1+\Gamma^{2}}}-1\right] \\
b_{0} & =6\left[\frac{1}{1+\Gamma^{2}}-\frac{1}{\sqrt{-\Gamma^{2}}} \operatorname{artanh}\left(\sqrt{-\Gamma^{2}}\right)\right]+2 \\
b_{1} & =\frac{6}{\sqrt{-\Gamma^{2}}} \operatorname{artanh}\left(\sqrt{-\Gamma^{2}}\right)-3 \\
b_{2} & =\frac{12}{1+\Gamma^{2}} \\
\sigma & =\frac{1}{\Gamma^{2}}\left(2-b_{0}\right)
\end{aligned}
$$

We see, that if $\Gamma^{2}$ approaches -1 the heat kernel blows up indicating violation of the strong ellipticity condition.

As an example, let us consider the open string sigma model of sec. 3.2. To simplify the subsequent calculations we put $B_{A B}=0$. In the zeta function regularization divergent part of the effective action $(2.31)$ reads $(1 / s) a_{2}(D)=$ $(1 / s) a_{2}(1, D)$. On general grounds we expect that $a_{2}$ repeats the structure of the classical action (3.10):

$$
a_{2}=\int_{\partial M} d \tau \beta_{A}^{[A]} \partial_{\tau} \bar{X}^{A}+\text { bulk terms, }
$$


where $\beta_{A}^{[A]}$ is a beta function ${ }^{20}$. We put $L_{a b}=0$ (otherwise we have had to introduce a dilaton coupling on the boundary to achieve renormalizability). We also suppose that the target space metric $G_{A B}$ is trivial. With these simplifying assumptions the beta function can be easily calculated from (5.38), (5.36), (5.37), (3.21):

$$
\beta_{C}^{[A]}=-\frac{1}{2 \pi}\left(\partial_{A} F_{B C}\right)\left(1+F^{2}\right)_{B A}^{-1} .
$$

By a lengthy but straightforward calculation one can demonstrate that the condition $\beta_{C}^{[A]}=0$ is equivalent to the equations of motion following from the Born-Infeld action on the target space:

$$
\mathcal{L}_{\mathrm{BI}}=\int d \bar{X} \sqrt{\operatorname{det}(1+i F)}=\int d \bar{X} \exp \left(\frac{1}{4} \operatorname{tr} \ln \left(1+F^{2}\right)\right)
$$

where $i$ appeared due to our rule of the Euclidean rotation for the gauge fields.

The Born-Infeld action has been derived from the beta functions of the open string sigma model in [1,112] confirming an earlier work [212] which used different methods. The heat kernel analysis was performed by Osborn [352] and then repeated in [306] for more general couplings.

\subsubsection{Spectral or Atiyah-Patodi-Singer (APS) boundary conditions}

Spectral boundary condition were introduced by Atiyah, Patodi and Singer in their study of the Index Theorem [19,20,21]. These boundary conditions are global, i.e. they cannot be defined by using local data only.

Consider a Dirac type operator

$$
\not D=i \gamma^{\mu} \tilde{\nabla}_{\mu}+\mathcal{E}
$$

where $\tilde{\nabla}$ is a covariant derivative with a compatible connection:

$$
\tilde{\nabla}_{\mu} \gamma_{\nu}=0
$$

i.e., the gamma matrices are covariantly constant. $\mathcal{E}$ is a zeroth order operator (a matrix valued function). We also suppose that the connection in $\tilde{\nabla}$ is unitary. This means that the connection one-form is represented by an antihermitian matrix in a suitable basis. We restrict ourselves to the case when $\mathcal{E}^{\dagger}=\mathcal{E}$, so that the operator $\not D$ is formally self-adjoint in the bulk. Note, that compatible unitary connection is not unique. Consider a first order differential operator on the boundary:

$$
P=\gamma_{n} \gamma^{a} \tilde{\nabla}_{a}+\frac{i}{2}\left(\mathcal{E} \gamma_{n}-\gamma_{n} \mathcal{E}\right)+\Theta(x)
$$

$\overline{{ }^{20} \text { In this }}$ case the subscript $A$ is a target space vector index, while the superscript $[A]$ indicates the coupling. Here we do not consider other beta functions $\beta^{[G]}, \beta^{[B]}$ etc. 
where $\Theta(x)$ is a hermitian matrix valued function on $\partial M$. The operator $P$ is a self-adjoint operator of Dirac type on the boundary. All functions on $\partial M$ can be decomposed in positive, negative, and zero modes of $P$. Let us define $\Pi_{-}$ as a projector on the space spanned by non-negative eigenspaces of $P$. Then the equation

$$
\left.\Pi_{-} \phi\right|_{\partial M}=0
$$

defines the APS boundary conditions.

Of course, relative complexity and non-locality limits physical applications of spectral boundary conditions. However, they appeared in a number of axial anomaly calculations [369, 280,278, 342,341,312,208,201], quantum cosmology [142], and in works on the Aharonov-Bohm effect [327,61]. In brane models spectral boundary conditions describe T-selfdual configurations which may be interpreted as mixtures of D-branes and open strings [406].

General form of the heat kernel expansion for spectral boundary conditions was established by Grubb and Seeley [260,261, 262]:

$$
K(t, f, D) \simeq \sum_{k=0}^{n-1} a_{k} t^{(k-n) / 2}+\sum_{j=n}^{\infty}\left(a_{j}^{\prime} \ln t+a_{j}^{\prime \prime}\right) t^{(j-n) / 2}
$$

In the contrast to all previous cases the expansion (5.45) contains logarithms of the proper time $t$. Although, such terms appear "typically" [241] for pseudo-differential operators, they may lead to rather unpleasant physical consequences. As follows from (2.25), non-zero $a_{n}^{\prime}$ means that the zeta function has a pole at $s=0$, and, therefore, the expression (2.32) for the renormalised effective action does not make sense. Fortunately, for the APS boundary conditions $a_{n}^{\prime}=0$ if $f=1$ near the boundary. Consequently, the integrated zeta function is regular at $s=0$ and eq. (2.32) still can be used. However, all calculations involving localised heat kernel coefficients remain problematic. Many logarithmic terms vanish if the manifold $M$ has a product structure near $\partial M$. In non-product cases strong criteria of "partial vanishing of logarithms" have been found recently [263].

Another problem with the heat kernel expansion (5.45) is that the coefficients may have a more complicated dependence on $n$ than just a power ${ }^{21}$ of $(4 \pi)$. After this long list of troubles it is not a surprise that only the coefficients $a_{k}$ with $k<n$ are locally computable. The coefficient $a_{0}$ is given by (4.26). $a_{1}$ and $a_{2}$ have been calculated in [172]:

\footnotetext{
${ }^{21}$ Let us remind that to prove the simple dependence on $n$ we used the product formulae (4.3), (4.4). We assumed that the spectral problem can be "trivialised" in one direction. In the present case all tangential coordinates enter the operator (5.43) on equal footings. Therefore, the proof does not go through.
} 


$$
\begin{aligned}
& a_{1}=(4 \pi)^{(1-n) / 2} \frac{1}{4}(\beta(n)-1) \int_{\partial M} d^{n-1} x \sqrt{h} \operatorname{tr}_{V}(f) \\
& a_{2}=(4 \pi)^{-n / 2}\left[\int_{M} d^{n} x \sqrt{g} \operatorname{tr}_{V}\left(f\left(\frac{1}{6} R+E\right)\right)\right. \\
& \quad+\int_{\partial M} d^{n-1} x \sqrt{h} \operatorname{tr}_{V}\left(f \mathcal{E}+\frac{1}{3}\left(1-\frac{3}{4} \pi \beta(n)\right) L_{a a} f\right. \\
&\left.\left.\quad-\frac{n-1}{2(n-2)}\left(1-\frac{1}{2} \pi \beta(n)\right) f_{; n}\right)\right]
\end{aligned}
$$

where

$$
\beta(n)=\Gamma\left(\frac{n}{2}\right) \Gamma\left(\frac{1}{2}\right)^{-1} \Gamma\left(\frac{n+1}{2}\right)^{-1} .
$$

The coefficient $a_{3}$ can be found in [242].

Some string theory applications suggest $[406,414]$ that spectral boundary conditions can be defined directly for a second order differential operator. Existence of the asymptotic expansion (5.45) and vanishing of leading logarithms for such problems have been stated in [264]. 


\section{Manifolds with singularities}

All results on the heat kernel expansion formulated in the previous sections are valid on smooth manifolds only. If there are boundaries, they also have to be smooth. As well, any singularities in the potential term or in the field strength are strictly speaking forbidden. However, many physical models deal with singular backgrounds. Even if such backgrounds may be represented through certain limiting procedures from smooth configurations, the heat kernel coefficients are not given by limits of their "smooth" values. The most visible manifestation of failure of the smooth field approximation is that $a_{k}$ with sufficiently large $k$ are divergent. Usually, the presence of singularities changes even the structure of the heat kernel kernel expansion as compared to the smooth case.

\section{$6.1 \quad$ Non-integrable potentials}

According to (1.21) divergences in the effective action are defined by integrated heat kernel coefficients. Although the formulae (4.26) - (4.29) for the localised heat kernel coefficients are valid on non-compact manifolds (provided the smearing function $f$ falls off sufficiently fast), transition to the integrated heat kernel is not that straightforward. Already the coefficient $a_{0}(D)=a_{0}(1, D)$, which is proportional to the volume, is divergent. This divergence is usually removed by replacing $\operatorname{det}(D)$ in (1.6) by

$$
\operatorname{det}(D) / \operatorname{det}\left(D_{0}\right)
$$

where the operator $D_{0}=-\partial^{2}+m^{2}$ describes a free particle propagation in an "empty" space. It is argued that since $D_{0}$ does not depend on "essential" variables division by $\operatorname{det}\left(D_{0}\right)$ does not change physical predictions of the theory. In all subsequent formulae the heat kernel $K(t ; x, y ; D)$ is then replaced by the subtracted heat kernel

$$
K_{\text {sub }}(t ; x, y)=K(t ; x, y ; D)-K\left(t ; x, y ; D_{0}\right) .
$$

In flat space the coefficient $a_{0}$ corresponding to $K_{\text {sub }}$ is identically zero ${ }^{22}$. If the field strength $\Omega_{\mu \nu}$ and the (subtracted) potential $E+m^{2}$ have a compact support or decay sufficiently fast at the infinity, the small $t$ asymptotic expansion of $K_{\mathrm{sub}}(t ; x, x)$ is integrable on the whole $M$. If not, the very structure of the global heat kernel may be changed.

As an example of non-integrable potentials consider the harmonic oscillator in one dimension. The Schrödinger operator reads

$$
D=-\partial_{x}^{2}+\nu^{2} x^{2} .
$$

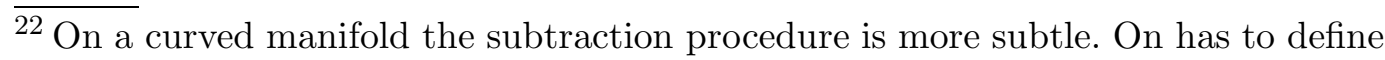
a reference metric which differs from the physical one on a compact submanifold. 
If we consider the problem on the whole real axis, $M=\mathbb{R}$, the potential term is not integrable. Already the expression $(4.27)$ for $a_{2}(1, D)$ diverges. Therefore, analytic expressions of sec. 4 cannot be used in this case. However, the (integrated) heat kernel can be easily calculated. Eigenvalues of the operator (6.3) are contained in almost any textbook on quantum mechanics:

$$
\lambda_{j}=\nu(2 j+1), \quad j=0,1,2, \ldots
$$

The integrated heat kernel reads

$$
K(t ; D)=\sum_{j=0}^{\infty} e^{-t \nu(2 j+1)}=\frac{1}{2}[\sinh (\nu t)]^{-1} .
$$

As $t \rightarrow 0$ it behaves like $1 / t$ while for smooth rapidly decaying potentials in one dimension the leading singularity in the heat kernel is $1 / \sqrt{t}$. This statement may be generalised to higher dimensions. If $D=-\partial^{2}+P_{\mu \nu} x^{\mu} x^{\nu}$ with a non-degenerate matrix $P_{\mu \nu}$ on $M=\mathbb{R}^{n}$, the leading term in $K(t ; D)$ is $(2 t)^{-n}(\operatorname{det} P)^{-1 / 2}[29]$.

\subsection{Conical singularities}

Conical space is defined as $M=[0,1] \times N$ where $N$ is an $n$-1-dimensional manifold called the base. The metric of the cone has the form

$$
(d s)^{2}=d r^{2}+r^{2} d \Omega^{2}
$$

where $r \in[0,1]$ and $d \Omega^{2}$ is the line element on the base $N$. This metric is, in general, singular at $r=0$. However, if we take the unit sphere $S^{n-1}=N$ with standard round metric, the singularity disappears and we obtain the $n$-dimensional unit ball (5.10). If a manifold has singular points where the metric can be approximated by (6.6) we say that this manifold has conical singularities.

Conical singularities appear in many physical applications. First of all, with $N=S^{1}$ the metric (6.6) is the Euclidean version of the Rindler metric. Conical singularities appear in classical solutions of the Einstein equations $[389,217,45]$ and in the supermembrane theory [400]. Gravitational field of a point mass in three dimensional gravity is a conical space $[146,145]$. There are evidences [376] that "conifolds" dominate the path integral for quantum gravity in topological sectors.

Sommerfeld [391] was probably the first to consider the heat kernel in the presence of conical singularities. The mathematical theory of the heat kernel asymptotics with conical singularities was developed almost 100 years later $[122,97,98]$. There two peculiar features of these asymptotics. First, the heat kernel expansion contains in general both integer and half-integer powers of $t$ even without boundaries. Second, a non-standard $\ln t$ term may 
be contained in the asymptotic series ${ }^{23}$.

On a manifold with conical singularities no closed analytical expression for the heat kernel coefficients is available. However, usually it is possible to disentangle contributions of the singularities from the smooth part. For example, if $N=S^{1}, d \Omega^{2}=d \varphi^{2}$ with $\varphi \in[0, \alpha]$ only $a_{2}$ receives a contribution from the tip of the cone:

$$
a_{2}(\operatorname{tip})=\frac{4 \pi^{2}-\alpha^{2}}{24 \pi \alpha}
$$

In many particular cases of conical singularities a very detailed analysis of the heat kernel expansion has been performed [154, 155, 121, 156, 226, 227, $129,130,230,85,140,385,169,66]$. One-loop computations on general orbifolds were considered recently in [255].

\subsection{Domain walls and brane world}

Delta function is an example of an extremely sharp background potential. Let us consider a manifold $M$ and a submanifold $\Sigma$ of the dimension $n-1$. Let

$$
D[v]=D+v \delta_{\Sigma}
$$

$D$ is an operator of Laplace type (2.2). Let $h$ be the determinant of the induced metric on $\Sigma$. Then $\delta_{\Sigma}$ is a delta function defined such that

$$
\int_{M} d x \sqrt{g} \delta_{\Sigma} f(x)=\int_{\Sigma} d x \sqrt{h} f(x) .
$$

The spectral problem for $D[v]$ on $M$ as it stands is ill-defined owing to the discontinuities (or singularities) on $\Sigma$. It should be replaced by a pair of spectral problems on the two sides $M^{ \pm}$of $\Sigma$ together with suitable matching conditions on $\Sigma$. In order to find such matching conditions, we consider an eigenfunction $\phi_{\lambda}$ of the operator (6.8):

$$
D[v] \phi_{\lambda}=\lambda \phi_{\lambda}
$$

Let us choose the coordinate system such that $e_{n}$ is a unit normal to $\Sigma$ and $x^{n}=0$ on $\Sigma$. It is clear that $\phi_{\lambda}$ must be continuous on $\Sigma$ :

$$
\left.\phi\right|_{x^{n}=+0}=\left.\phi\right|_{x^{n}=-0} .
$$

Otherwise, the second normal derivative of $\phi_{\lambda}$ would create a $\delta^{\prime}$ singularity on $\Sigma$ which is absent on the right hand side of (6.10). Let us integrate (6.10) over a small cylinder $\mathcal{C}=C^{n-1} \times[-\epsilon,+\epsilon]$

$$
\int_{\mathcal{C}} d^{n} x \sqrt{g}\left(-\nabla_{n}^{2} \phi_{\lambda}-\left[\nabla_{a}^{2} \phi_{\lambda}+(E+\lambda) \phi_{\lambda}\right]\right)+\int_{C} d^{n-1} x \sqrt{h} v \phi_{\lambda}=0 .
$$

$\overline{23}$ The present author is not aware of any simple example where the $\ln t$ terms actually appear. 
We now take the limit as $\epsilon \rightarrow 0$. Since the expression in the square brackets in (6.12) is bounded, the contribution that this term makes vanishes in the limit. We obtain

$$
0=\int_{C} d^{n-1} x \sqrt{h}\left(-\left.\nabla_{n} \phi_{\lambda}\right|_{x^{n}=+0}+\left.\nabla_{n} \phi_{\lambda}\right|_{x^{n}=-0}+v \phi_{\lambda}\right) .
$$

Since $C$ and $\lambda$ are arbitrary, we conclude that a proper matching condition for the normal derivatives is

$$
-\left.\nabla_{n} \phi\right|_{x^{n}=+0}+\left.\nabla_{n} \phi\right|_{x^{n}=-0}+v \phi=0 .
$$

Physically this problem corresponds to two domains separated by a penetrable membrane $\Sigma$ (a domain wall). In many cases penetrable membranes are better models of physical boundaries then just boundary conditions which are imposed on each side of $\Sigma$ independently and thus exclude any interaction between the domains $[285,2] . \delta$-potentials are being used in quantum mechanics [6] where one studies the Schrödinger equation (which is nothing else than the imaginary time heat equation). The Casimir energy calculations have been performed e.g. in [374]. In the formal limit $v \rightarrow \infty$ one obtains Dirichlet boundary conditions on $\Sigma$, although the heat kernel coefficients are divergent in this limit (see below).

Further generalisations are suggested by the brane world scenario [363, 362] which assumes that our world is a four dimensional membrane in a five dimensional space ${ }^{24}$. According to the Israel junction condition [283] the metric in such models cannot be smooth on $\Sigma$. Typical form of the metric near $\Sigma$ is

$$
(d s)^{2}=\left(d x^{n}\right)^{2}+e^{-\alpha\left|x^{n}\right|}\left(d s_{n-1}\right)^{2},
$$

where $\alpha$ is a constant and where $\left(d s_{n-1}\right)$ is a line element on the $(n-1)$ dimensional hypersurface $\Sigma$. Due to the presence of the absolute value of the $n$-th coordinate in (6.15), the normal derivative of the metric jumps on $\Sigma$. One can think of two smooth manifolds $M^{+}$and $M^{-}$glued together along their common boundary $\Sigma$. Neither Riemann tensor, nor matrix potential $E$ must be continuous on $\Sigma$. Also, the extrinsic curvatures $L_{a b}^{+}$and $L_{a b}^{-}$of $\Sigma$ considered as a submanifold in $M^{+}$and in $M^{-}$respectively are, in general, different. All geometric quantities referring to $M^{-}$(respectively, $M^{-}$) and their limiting values on $\Sigma$ will be supplied by a superscript "+" (respectively, "-").

For the case at hand there is still an asymptotic expansion (2.21) for the heat kernel. The heat kernel coefficients can be decomposed as

$$
a_{k}(f, D[v])=a_{k}^{+}(f, D)+a_{k}^{-}(f, D)^{-}+a_{k}^{\Sigma}(f, D, v)
$$

where $a_{k}^{ \pm}(f, D)$ are known volume contributions corresponding to $M^{ \pm}$(cf. (4.26) - (4.29)). The coefficients $a_{k}^{\Sigma}$ are given by integrals over $\Sigma$ of some local

\footnotetext{
${ }^{24}$ A similar scenario was proposed earlier in [371], see [370] for a review.
} 
invariants. Note, that

$$
\tilde{\omega}_{a}=\nabla_{a}^{+}-\nabla_{a}^{-}
$$

being a difference of two connection is a (pseudo-) vector with respect to all space-time and gauge symmetries. Therefore, $\tilde{\omega}_{a}$ can be used for constructing the surface invariants.

To make the formulae more symmetric we introduce two inward pointing unit normals $\nu^{+}$and $\nu^{-}$to $\Sigma$ in $M^{+}$and $M^{-}$respectively. We do not suppose that the smearing function $f$ is smooth on $\Sigma$ (therefore, there is no relation between $f_{; \nu^{+}}$and $f_{; \nu^{-}}$), but we assume continuity of $f: f^{+}=f^{-}=f$ on $\Sigma$.

The surface invariants can be constructed from $L_{a b}^{ \pm}, R_{i j k l}^{ \pm}, E^{ \pm}, v, \tilde{\omega}_{a}$ and their derivatives. This gives much more invariants than we usually have for a boundary value problem. There are, however, some properties of $a_{k}^{\Sigma}$ which simplify the calculations considerably. First of all, $a_{k}^{\Sigma}$ must be invariant with respect to interchanging the roles of $M^{+}$and $M^{-}$. Also, $a_{k}^{\Sigma}$ must vanish when the singularity disappears. The first property excludes, for example, the term $f\left(E^{+}-E^{-}\right)$which changes sign under $M^{+} \leftrightarrow M^{-}$. The second requirement excludes the invariant $f\left(E^{+}+E^{-}\right)$because it survives even if there is no singularity on $\Sigma$. These simple arguments show that $a_{3}^{\Sigma}$ does not contain $E^{ \pm}$ even though such terms are allowed on dimensional grounds. It is also very helpful that in some particular cases the problem in question can be reduces to a sum of Dirichlet and Robin boundary value problems $[82,248]$.

The coefficients $a_{k}^{\Sigma}, k=0,1,2,3$, read

$$
\begin{aligned}
& a_{0}^{\Sigma}(f, D, v)=0 . \\
& a_{1}^{\Sigma}(f, D, v)=0 . \\
& a_{2}^{\Sigma}(f, D, v)=(4 \pi)^{-n / 2} \frac{1}{6} \int_{\Sigma} d^{n-1} x \sqrt{h} \operatorname{tr}_{V}\left\{2 f\left(L_{a a}^{+}+L_{a a}^{-}\right)-6 f v\right\} . \\
& a_{3}^{\Sigma}(f, D, v)=(4 \pi)^{(1-n) / 2} \frac{1}{384} \int_{\Sigma} d^{n-1} x \sqrt{h} \operatorname{tr}_{V}\left\{\frac { 3 } { 2 } f \left(L_{a a}^{+} L_{b b}^{+}+L_{a a}^{-} L_{b b}^{-}\right.\right. \\
&\left.\quad+2 L_{a a}^{+} L_{b b}^{-}\right)+3 f\left(L_{a b}^{+} L_{a b}^{+}+L_{a b}^{-} L_{a b}^{-}+2 L_{a b}^{+} L_{a b}^{-}\right) \\
& \quad+9\left(L_{a a}^{+}+L_{a a}^{-}\right)\left(f_{; \nu^{+}}^{+}+f_{; \nu^{-}}^{-}\right)+48 f v^{2}+24 f \tilde{\omega}_{a} \tilde{\omega}_{a} \\
&\left.\quad-24 f\left(L_{a a}^{+}+L_{a a}^{-}\right) v-24\left(f_{; \nu^{+}}^{+}+f_{; \nu^{-}}^{-}\right) v\right\} .
\end{aligned}
$$

The coefficients $a_{4}^{\Sigma}$ and $a_{5}^{\Sigma}$ are too long to be presented here in full generality. Therefore, we restrict ourselves to the case of smooth geometry $\left(R_{i j k l}^{+}=R_{i j k l}^{-}\right.$, $\left.L_{a b}^{+}=-L_{a b}^{-}\right)$, smooth connection $\left(\tilde{\omega}_{a}=0\right)$, and smooth smearing function $\left(f_{; \nu^{+}}=-f_{; \nu^{-}}=f_{; n}\right)$. In other words, the only singularity comes from the surface potential $v$. 


$$
\begin{aligned}
& a_{4}^{\Sigma}(f, D, v)=(4 \pi)^{-n / 2} \int_{\Sigma} d^{n-1} x \sqrt{h} \operatorname{tr}_{V}\left\{-\frac{1}{6} f v^{3}-\frac{1}{6} f R v-f E v\right. \\
& \left.\quad-\frac{1}{6} f v_{: a a}+\frac{1}{6} f_{; n} v L_{a a}-\frac{1}{6} f_{; n n} v\right\} \\
& a_{5}^{\Sigma}(f, D)=(4 \pi)^{-(n-1) / 2} \int_{\Sigma} d^{n-1} x \sqrt{h} \operatorname{tr}_{V}\left\{\frac{1}{64} f v^{4}+\frac{1}{48} f R v^{2}\right. \\
& \quad+\frac{1}{192} R_{n n} v^{2}+\frac{1}{8} f v^{2} E-\frac{1}{256} f v^{2} L_{a a} L_{b b}+\frac{1}{128} f v^{2} L_{a b} L_{a b} \\
& \left.+\frac{1}{24} f v_{: a a} v+\frac{5}{192} f v_{: a} v_{: a}-\frac{5}{384} f_{; n} v^{2} L_{a a}+\frac{1}{64} f_{; n n} v^{2}\right\}
\end{aligned}
$$

The heat kernel coefficients for $\Sigma=S^{n-1} \subset \mathbb{R}^{n}$ and $v=$ const. were calculated in [77]. Generic $\Sigma$ with arbitrary $v$ was considered in [82]. Moss [330] added a non-smooth connection (see also [173]). Calculations on a particular brane-world background can be found in [343]. The heat kernel coefficients in the general setting described here were calculated in [248]. This latter paper also considered renormalization of the brane-world scenario and predicted a non-standard Higgs potential on the brane. Related calculations in wormhole models were done in $[294,293]$. The $\zeta$ function for brane-world geometries with matching conditions (6.11), (6.14) was considered recently in [186,332] (where one can find some further references).

It is very well known [6] that the conditions (6.11), (6.14) (which we call transmittal) are not the most general matching conditions which can be defined on a surface. In general, boundary values of a function and of its' normal derivatives are related by a $2 \times 2$ transfer matrix:

$$
0=\left.\left(\begin{array}{cc}
\nabla_{\nu^{+}}^{+}+S^{++}, & S^{+-} \\
S^{-+}, & \nabla_{\nu^{-}}^{-}+S^{--}
\end{array}\right)\left(\begin{array}{c}
\phi^{+} \\
\phi^{-}
\end{array}\right)\right|_{\Sigma}
$$

Note, that the transfer conditions (6.20) do not assume identification of $\phi^{+}$and $\phi^{-}$on $\Sigma$. In other words, there is no ad hoc relation between the restrictions of the vector bundles $\left.V^{+}\right|_{\Sigma}$ and $\left.V^{-}\right|_{\Sigma}$. We can even consider the situation when we have $\operatorname{dim} V^{+} \neq \operatorname{dim} V^{-}$, i.e. the fields on $M^{-}$and $M^{-}$can have different structures with respect to space-time and internal symmetries. $S^{ \pm \pm}$are some matrix valued functions on $\Sigma$ (one can even consider the case when they are differential operators). It is clear from the notations on which spaces they act. For example, $S^{+-}:\left.\left.V^{-}\right|_{\Sigma} \rightarrow V^{+}\right|_{\Sigma}$.

The matching conditions (6.20) arise in heat transfer problems [119], some problems of quantum mechanics [256], and in conformal field theory [40]. In a formal limiting case $S^{++}-S^{-+}=S^{--}-S^{+-} \rightarrow \infty$ while $v=2\left(S^{++}+S^{+-}\right)$is kept finite one arrives at transmittal boundary conditions (6.11), (6.14). The heat kernel coefficients are divergent in this limit.

In a particular case of spherical $\Sigma$ the heat kernel expansion with transfer boundary conditions was evaluated in [81]. General expressions for $a_{k}$ with $k=0,1,2,3,4$ were obtained in [247]. Somewhat surprisingly, the calculations 
for (6.20) are easier than for the singular particular case (6.11), (6.14).

We should note that not all singular limiting cases of (6.20) are described by the transmittal conditions (6.11), (6.14). The heat kernel expansion for a generalisation of transmittal condition is known in the spherically symmetric case only [81]. Very little is known about the heat kernel if the transfer matrix contains differential operators on $\Sigma$ (conformal walls of ref. [40] belong to this class of problems).

The case when the singular potential is located on a surface of co-dimension larger than one (i.e. when $\operatorname{dim} \Sigma<n-1$ ) is rather complicated. Even a careful translation of this problem to the operator language was done only in 1960's [63]. Direct calculations show [5,390] that the heat kernel asymptotics may contain very unusual $(\ln t)^{-1}$ terms. More references can be found in [114].

\subsection{Non-smooth boundaries}

Rectangular region in a plane is probably the simplest manifold with boundaries as far as eigenvalues of the Laplacian are concerned. However, the formulae (5.29) - (5.33) are not valid for this case because of the presence of corners. The heat kernel expansion on manifolds with piecewise smooth boundaries was considered by Kac in his famous paper [286]. He demonstrated that for a region in $\mathbb{R}^{2}$ each corner with the inside facing angle $\alpha$ contributes

$$
a_{2}(\text { corner })=\frac{\pi^{2}-\alpha^{2}}{24 \pi \alpha}
$$

to the coefficient $a_{2}$ while $a_{0}$ and $a_{1}$ are still defined by their "smooth" expressions.

The formula (6.21) looks similar to the contribution of a conical singularity (6.7). The reason for this similarity is that the cone can be obtained from the wedge by gluing the sides together and imposing the periodicity conditions.

The study of boundary discontinuities was continued by Apps and Dowker who calculated the coefficients $a_{3}$ [163] and $a_{4}$ [17] for piecewise smooth boundaries. We also refer to [23] where functional determinants on simplicial complexes were analysed, and to [161] where divergences in the Casimir energy found in [338] were attributed to non-smoothness of the boundaries. A recent study [340] should also be mentioned.

When the angle $\alpha$ goes to 0 we obtain a cusp. In this limit (6.21) is divergent. Presence of the cusp is an essential singularity which modifies powers of the proper time $t$ which appear in the asymptotic expansion of the heat kernel [392].

\subsection{Dielectric bodies}

Calculations of the Casimir energy of a dielectric body have attracted

much attention and created many controversial results. A (rather large) liter- 
ature on this subject is reviewed in [79,322]. Quantum field theory formulation of this problem is known for a long time already (cf. [380,323,116]). However, the heat kernel analysis of divergences in the Casimir energy in dielectric is a relatively new subject.

Wave propagation with variable speed of light $c(x)$ is described by the operator $D=-c(x)^{2} \nabla^{2}+\ldots$, where we neglected the lower order terms. In a dielectric medium $c$ is expressed in terms of the dielectric permittivity $\varepsilon$ and of the magnetic permeability $\mu: c(x)^{2}=1 /(\varepsilon(x) \mu(x))$. For a smooth distribution of $\varepsilon$ and $\mu$ the operator $D$ is a particular case of curved space Laplacian with an effective metric defined by $c(x)$. The heat kernel coefficients can be calculated in the standard way [78].

Consider now a dielectric body bounded by $\Sigma$. Typically, $c(x)$ (and the effective metric!) jumps on $\Sigma$. This singularity is much stronger than the one considered in sec. 6.3. Thus the geometric interpretation of this problem is very difficult. Very little is known about the heat kernel expansion in a dielectric body of an arbitrary shape. There are calculations for a dielectric ball [77] and for a dielectric cylinder [80]. These calculations exhibit a puzzling property of the heat kernel expansion in dielectrics in dilute approximation $(\varepsilon \sim 1)$ : for a dielectric body the Casimir energy in the ultra violet limit behaves better than for the "smooth" case. The heat kernel expansion for a frequency dependent $\varepsilon$ was considered in refs. [202,76]. 


\section{Anomalies}

The most immediate application of the technique developed in the previous sections is calculation of quantum anomalies which are defined as (nonzero) variations of quantum effective action with respect to symmetry transformations of the classical theory. In this section we consider two most important examples of quantum anomalies. These are conformal (scale) and chiral anomalies. We also discuss briefly the Index Theorem.

There exists also a broader view on the anomalies which includes any qualitatively new phenomena of a quantum theory which are absent in its' classical counterpart. An example is the so-called dimensional reduction anomaly [216]. For two operators $D_{1}$ and $D_{2}$ the quantity $\operatorname{det}\left(D_{1}\right) \operatorname{det}\left(D_{2}\right) / \operatorname{det}\left(D_{1} D_{2}\right)$ is called the multiplicative anomaly [187] since $\operatorname{det}\left(D_{1} D_{2}\right) \neq \operatorname{det}\left(D_{1}\right) \operatorname{det}\left(D_{2}\right)$ is an "anomalous" property of infinite dimensional operators. It is interesting to note that the heat kernel is also useful for treatment of these non-standard anomalies.

To simplify the discussion in this section we work on manifolds without boundaries.

\subsection{Conformal anomaly}

Conformal invariance is one of the symmetries which are usually broken by quantisation. This phenomenon (called conformal or trace anomaly) is known since mid 1970's [118,144,165,96,398] (see [69,174] for more extensive literature).

The vacuum polarisation induced by quantum effects is described by the energy-momentum tensor

$$
T_{\mu \nu}=\frac{2}{\sqrt{g}} \frac{\delta W}{\delta g^{\mu \nu}},
$$

where $W$ is the quantum effective action calculated on the background with the metric tensor $g_{\mu \nu}$. Consider the conformal transformation

$$
g_{\mu \nu} \rightarrow e^{2 \rho(x)} g_{\mu \nu}
$$

for an infinitesimal value of the parameter: $g_{\mu \nu} \rightarrow(1+2 \delta \rho) g_{\mu \nu}, g^{\mu \nu} \rightarrow(1-$ $2 \delta \rho) g^{\mu \nu}$. According to (7.1) variation of the effective action reads:

$$
\delta W=\frac{1}{2} \int_{M} d^{n} x \sqrt{g} T_{\mu \nu} \delta g^{\mu \nu}=-\int_{M} d^{n} x \sqrt{g} T_{\mu}^{\mu} \delta \rho .
$$

It is clear from the equation above that the trace of the energy-momentum tensor measures conformal non-invariance of the theory. If the classical action $\mathcal{L}$ is conformally invariant, classical energy-momentum tensor is traceless. However, even in this case conformal invariance is typically broken by quantum effects. For this reason, quantum $T_{\mu}^{\mu}$ is called trace or conformal anomaly. 
If the classical action $\mathcal{L}$ is conformally invariant, the fluctuation operator $D$ is conformally covariant. This means that $D$ transforms homogeneously $D \rightarrow e^{-2 \rho} D$ under $(7.2)^{25}$.

We restrict ourselves to the one-loop level and employ the zeta function regularization in which the effective action is expressed through the zeta function of the operator $D$ (see (2.32)). Hence, we have to study the conformal properties of $\zeta(s, D)$. One can prove that the variation of the zeta function with respect to variation of the operator $D$ reads ${ }^{26}$

$$
\delta \zeta(s, D)=-s \operatorname{Tr}\left((\delta D) D^{-s-1}\right)
$$

Next we use that under infinitesimal conformal transformations the operator $D$ transforms as

$$
\delta D=-2(\delta \rho) D
$$

This equation yields

$$
\delta \zeta(s, D)=2 s \zeta(s, \delta \rho, D) .
$$

For the operators which we consider in this section the zeta function is regular at $s=0$. Consequently, the variation of the effective action (2.32) reads:

$$
\delta W=-\zeta(0, \delta \rho, D)=-a_{n}(\delta \rho, D)
$$

where we have used the relation (2.27) between the zeta function at zero argument and the heat kernel coefficient $a_{n}$.

We compare (7.3) with (7.7) to see that

$$
T_{\mu}^{\mu}(x)=a_{n}(x, D) .
$$

Note, that $\zeta(0, D)$ (as well as the pole term in $(2.31)$ ) is conformally invariant. Consequently, the conformal anomaly is not divergent, and (7.8) does not contain the normalisation scale $\mu$.

The anomaly (7.8) is defined by the same coefficient $a_{n}$ as the divergent part of the effective action in the zeta function regularization. Important difference is that the divergence is given by the integrated coefficient $a_{n}(D)$, while the conformal anomaly is defined by the localised coefficient $a_{n}(x, D)$. The use of the integrated coefficient in (7.8) is insufficient to recover total derivatives in the anomaly.

\footnotetext{
${ }^{25}$ Strictly speaking this property holds up to a similarity transformation $D \rightarrow$ $e^{\alpha \rho} D e^{-\alpha \rho}$ which does not change the functional determinant.

26 To derive this formula rigorously we have to use the method of $[21,368]$. We first assume that $s$ is sufficiently large to keep us away from the singularities, then use the Mellin transformation (2.24), perform the variation, then perform the transformation back, and then continue the result to $s=0$. This is a perfectly standard procedure which allows us to work with variations of positive integer powers of $D$; see [198] for further detail.
} 
As an example, consider quantum scalar field $\phi$ in two dimensions ${ }^{27}$ coupled to the background dilaton $\Phi$ :

$$
\mathcal{L}=\int d^{2} x \sqrt{g} e^{-2 \Phi}\left(\partial_{\mu} \phi\right)\left(\partial_{\nu} \phi\right) g^{\mu \nu}
$$

On dimensional and symmetry grounds the inner product may also contain an arbitrary function $\Psi$ of the dilaton:

$$
\left\langle\phi_{1}, \phi_{2}\right\rangle=\int d^{2} x \sqrt{g} e^{-2 \Psi} \phi_{1}(x) \phi_{2}(x) .
$$

Such couplings and inner products appear, for example, after the spherical reduction of higher dimensional theories to two dimension (see the review paper [265] for more details and further references).

The rescaled field $\tilde{\phi}=e^{-\Psi} \phi$ possesses the standard dilaton-independent inner product (3.3). In terms of $\tilde{\phi}$ the action (7.9) reads

$$
\begin{aligned}
\mathcal{L}=\int d^{2} x \sqrt{g} \tilde{\phi} D \tilde{\phi} & \\
D=-e^{2(\Psi-\Phi)} g^{\mu \nu} & \left(\nabla_{\mu} \nabla_{\nu}+2\left(\Psi_{, \mu}-\Phi_{, \mu}\right) \partial_{\nu}\right. \\
& \left.\quad+\Psi_{, \mu \nu}+\Psi_{, \mu} \Psi_{, \nu}-2 \Psi_{, \mu} \Phi_{, \nu}\right)
\end{aligned}
$$

where comma denotes covariant differentiation with $\nabla: \Psi_{, \nu}=\nabla_{\nu} \Psi$. We can bring $D$ to the standard form $(2.2)$

$$
D=-\left(\hat{g}^{\mu \nu} \hat{\nabla}_{\mu} \hat{\nabla}_{\nu}+E\right)
$$

by introducing the effective metric $\hat{g}^{\mu \nu}=e^{2(\Psi-\Phi)} g^{\mu \nu}$ and the covariant derivative

$$
\hat{\nabla}_{\mu}=\partial_{\mu}+\hat{\Gamma}_{\mu}+\hat{\omega}_{\mu}, \quad \hat{\omega}_{\mu}=\Psi_{, \mu}-\Phi_{, \mu},
$$

where $\hat{\Gamma}$ is the Christoffel connection for the metric $\hat{g}$. Here the potential $E$ reads

$$
E=\hat{g}^{\mu \nu}\left(-\Phi_{, \mu} \Phi_{, \nu}+\Phi_{, \mu \nu}\right) .
$$

Now we combine (7.8) with (4.27) and the definitions given above to obtain:

$$
T_{\mu}^{\mu}=\frac{1}{24 \pi}\left(R-6(\nabla \Phi)^{2}+4 \nabla^{2} \Phi+2 \nabla^{2} \Psi\right) .
$$

In the case $\Psi=\Phi$ the expression (7.16) was first obtained in [336], and in the general case in $[303]^{28}$.

\footnotetext{
$\overline{{ }^{27} \mathrm{Much}}$ work on conformal anomalies on two-dimensional manifolds was done in the context of string theory. Here we like to mention the papers $[10,215,214,113,11]$. ${ }^{28}$ Different values for the numerical coefficients in the conformal anomaly (7.16) were reported in $[86,344,321,345]$. The reason for these discrepancies was clearly stated by Dowker [160] who confirmed the result (7.16). More extensive literature can be found in [265].
} 
In four dimensions the conformal anomaly for different spins can be read off from (4.35) with the numerical coefficients given in Table 1.

\subsection{Chiral anomaly}

Chiral anomaly was discovered in 1969 by Adler, Bell and Jackiw [3, 60] and since that time plays a crucial role in understanding of the low energy hadron physics. A detailed introduction to the field and extensive literature can be found in $[65,428]$.

The spinor action (3.22) with the Dirac operator given in (3.26) is invariant under the gauge transformations

$$
\begin{aligned}
& \delta_{\lambda} \psi=-\lambda \psi, \quad \delta_{\lambda} \bar{\psi}=\bar{\psi} \lambda, \\
& \delta_{\lambda} A_{\mu}^{5}=\left[A_{\mu}^{5}, \lambda\right], \\
& \delta_{\lambda} A_{\mu}=\partial_{\mu} \lambda+\left[A_{\mu}, \lambda\right]
\end{aligned}
$$

and local (Euclidean) chiral transformations

$$
\begin{aligned}
& \tilde{\delta}_{\varphi} \psi=-i \varphi \gamma^{5} \psi, \quad \tilde{\delta}_{\varphi} \bar{\psi}=-i \bar{\psi} \varphi \gamma^{5}, \\
& \tilde{\delta}_{\varphi} A_{\mu}^{5}=\partial_{\mu} \varphi+\left[A_{\mu}, \varphi\right], \\
& \tilde{\delta}_{\varphi} A_{\mu}=-\left[A_{\mu}^{5}, \varphi\right]
\end{aligned}
$$

with anti-hermitian local matrix parameters $\lambda$ and $\varphi$. The Dirac operator transforms as:

$$
\delta_{\lambda} \not D=[\not D, \lambda], \quad \tilde{\delta}_{\varphi} \not D=i\left\{\varphi \gamma^{5}, \not D\right\} .
$$

The Lie algebra structure of the transformations (7.17) and (7.18) is encoded in the following relations:

$$
\begin{aligned}
& \delta_{\lambda_{1}} \delta_{\lambda_{2}}-\delta_{\lambda_{2}} \delta_{\lambda_{1}}=\delta_{\left[\lambda_{1}, \lambda_{2}\right]} \\
& \delta_{\lambda} \tilde{\delta}_{\varphi}-\tilde{\delta}_{\varphi} \delta_{\lambda}=\tilde{\delta}_{[\lambda, \varphi]} \\
& \tilde{\delta}_{\varphi_{1}} \tilde{\delta}_{\varphi_{2}}-\tilde{\delta}_{\varphi_{2}} \tilde{\delta}_{\varphi_{1}}=-\delta_{\left[\varphi_{1}, \varphi_{2}\right]}
\end{aligned}
$$

where all transformation parameters are taken at the same space-time point. If the matrices $\lambda(x)$ and $\varphi(x)$ belong to a finite-dimensional compact Lie algebra of some Lie group $\mathcal{G}$, the transformations (7.17) and (7.18) generate locally the group $\mathcal{G} \times \mathcal{G}$ with gauge transformations belonging the diagonal sub-group.

The gauge invariance can (and should) be retained in quantum theory while the chiral invariance is typically broken by the quantisation. Let us study these effects in the zeta function regularization. Define determinant of the Dirac operator as a square root of the determinant of the associated Laplace operator:

$$
\log \operatorname{det} \not D=\frac{1}{2} \log \operatorname{det} \not D^{2}=\frac{1}{2} \log \operatorname{det} D
$$


The effective action $W$ corresponding to the path integral (3.41) reads

$$
W=-\log Z=-\frac{1}{2} \log \operatorname{det} D=\frac{1}{2} \zeta(0, D)^{\prime}+\frac{1}{2} \ln \left(\mu^{2}\right) \zeta(0, D),
$$

where $\mu$ is a normalisation scale.

By virtue of (7.4)

$$
\delta_{\lambda} \zeta(s, D)=-\left(s \operatorname{Tr}\left([D, \lambda] D^{-s-1}\right)\right)=\left(\operatorname{Tr}\left(\left[D^{-s}, \lambda\right]\right)\right)=0 .
$$

This proves gauge invariance of the effective action.

For the chiral transformation we have:

$$
\mathcal{A}(\varphi):=\tilde{\delta}_{\varphi} W=-\left.2 i \operatorname{Tr}\left(\gamma^{5} \varphi D^{-s}\right)\right|_{s=0} .
$$

$\mathcal{A}(\varphi)$ measures non-invariance of the effective action with respect to the chiral transformations. It is called the chiral anomaly. One can express $\mathcal{A}$ through the heat kernel coefficients:

$$
\mathcal{A}(\varphi)=-2 i a_{n}\left(\gamma^{5} \varphi, D\right),
$$

where $a_{n}\left(\gamma^{5} \varphi, D\right)$ is defined as in (2.21) but with a matrix-valued smearing function instead of the scalar one.

The chiral anomaly $\mathcal{A}(\varphi)$ should satisfy certain consistency conditions following from the Lie algebra identities (7.20)-(7.22) and gauge invariance of the effective action:

$$
\begin{aligned}
& \delta_{\lambda} \mathcal{A}(\varphi)=\mathcal{A}([\lambda, \varphi]), \\
& \tilde{\delta}_{\varphi_{1}} \mathcal{A}\left(\varphi_{2}\right)-\tilde{\delta}_{\varphi_{2}} \mathcal{A}\left(\varphi_{1}\right)=0 .
\end{aligned}
$$

The relations (7.28) and (7.28) are called the Wess-Zumino consistency conditions [417]. One can check by a direct calculation that the anomaly defined in this section indeed satisfies these conditions. For this reason $\mathcal{A}$ is called the consistent anomaly (as opposed to the covariant anomaly which we do not consider here).

Let us turn now to calculation of the chiral anomaly $\mathcal{A}$. Since the smearing function $\gamma^{5} \varphi$ in the heat kernel coefficient (7.27) is matrix-valued we need more information than we possess at the moment. To recover the missing terms one can adopt the strategy of the paper [94]. Consider $\operatorname{Tr}(Q \exp (-t D))$ with arbitrary matrix-valued function $Q$ and arbitrary Laplace type operator $D$. There is an asymptotic expansion

$$
\operatorname{Tr}_{L^{2}}(Q \exp (-t D)) \cong \sum_{k \geq 0} t^{(k-n) / 2} a_{k}(Q, D)
$$

where the coefficients $a_{k}$ are locally computable. This means that they can be represented as integrals of local invariants constructed from $Q$ and local invariants of the operator $D$. These local invariants enter with some coefficients 
(universal constants) which are to be defined. Since $Q$ does not commute with $E$ and $\Omega$ there are more invariants than before. In the particular case $Q=I_{V} f$ (where $I_{V}$ is the unit matrix and $f$ is a function) we should recover the old result $(4.26)-(4.29)$. For $k=0,2,4$ this last requirement is strong enough to recover $a_{k}(Q, D)$ completely. Therefore, calculation of the chiral anomaly in the dimensions $n=2$ and $n=4$ is quite simple: take the expression (4.27) or (4.28) and substitute $2 i \gamma^{5} \varphi$ for $f$ and (3.27), (3.28) for $\omega_{\mu}, E$ and $\Omega_{\mu \nu}$. Computation of some $\gamma$-matrix traces is still required, but nevertheless calculations are considerably easier than presented in many papers. In flat space and with $A^{5}=0$ the result is particularly simple:

$$
\begin{array}{ll}
n=2: & \mathcal{A}(\varphi)=-\frac{1}{2 \pi} \int d^{2} x \operatorname{tr}\left(\varphi \epsilon^{\mu \nu} F_{\mu \nu}\right) \\
n=4: & \mathcal{A}(\varphi)=\frac{i}{16 \pi^{2}} \int d^{4} x \operatorname{tr}\left(\varphi \epsilon^{\mu \nu \rho \sigma} F_{\mu \nu} F_{\rho \sigma}\right)
\end{array}
$$

where tr denotes trace over internal (flavour or colour) indices. We can easily generalise this result for arbitrary even dimension $n$. First we observe that the only way to construct a pseudoscalar density of appropriate dimension (for $\left.A^{5}=0\right)$ is to contract $n / 2$ tensors $F_{\mu \nu}$ with the Levi-Civita tensor $\epsilon^{\mu_{1} \mu_{2} \ldots \mu_{n}}$. Such tensor structure can be produced only by a trace of $\gamma^{5}$ and the maximal number $(n)$ of the gamma matrices. The only invariant having the required form is

$$
\int d^{n} x \operatorname{tr}\left(Q E^{n / 2}\right)
$$

This term does not contain derivatives. Under the trace $Q$ and $E$ commute. Therefore, we can calculate the coefficient in front of (7.33) by considering the particular case $E \sim I_{V} \times$ const.. For this simple case the dependence of the heat kernel on $E$ is given simply by $e^{t E}$. By picking up an appropriate term in the expansion of the exponential we find that the coefficient in front of (7.33) in $a_{n}$ is $(4 \pi)^{-n / 2}((n / 2) !)^{-1}$. Next we substitute $E=\frac{1}{2} \gamma^{\mu} \gamma^{\nu} F_{\mu \nu}$ and take trace over the spinorial indices to obtain:

$$
\mathcal{A}(\varphi)=-\frac{2 i(-i)^{n(n-1) / 2}}{(4 \pi)^{n / 2}(n / 2) !} \int d^{n} x \operatorname{tr}\left(\varphi \epsilon^{\mu_{1} \mu_{2} \ldots \mu_{n}} F_{\mu_{1} \mu_{2}} \ldots F_{\mu_{n-1} \mu_{n}}\right)
$$

For global chiral transformations $(\varphi=$ const.) this formula was obtained in [421].

As soon as we know the anomaly (7.34) for $A_{\mu}^{5}=0$, many more terms (containing $A_{\mu}^{5}$ ) can be restored by using the Wess-Zumino condition (7.29).

Some comments on the Fujikawa approach [220,221] to the chiral anomaly are in order. Consider the path integral (3.41). Since the action is invariant under the chiral rotations, the only source of the chiral anomaly could be noninvariance of the path integral measure. The Jacobian factor appearing due to the change of the spinorial variables (7.18) can be formally represented as

$$
J=\operatorname{det}\left(1-2 i \varphi \gamma^{5}\right)
$$


Then, to the first order of $\varphi$,

$$
\mathcal{A}(\varphi)=\tilde{\delta}_{\varphi} W=-\log J^{-1} \simeq-2 i \operatorname{Tr}\left(\varphi \gamma^{5}\right)
$$

where $J^{-1}$ has appeared due to the negative homogeneity of the fermionic measure. The operator $\varphi \gamma^{5}$ is not trace class on the space of square integrable spinors (though formally its' trace is zero at every point). The right hand side of (7.36) is therefore ill defined. Fujikawa suggested to replace (7.36) by a regularized expression

$$
\mathcal{A}(\varphi)=-2 i \lim _{M \rightarrow \infty} \operatorname{Tr}\left(\varphi \gamma^{5} e^{-D / M^{2}}\right) .
$$

The chiral anomaly (7.37) has now the heat-kernel form with the identification $t=M^{-2}$. If we suppose that all positive powers of the regularization parameter $M$ in the small $t$ (large $M$ ) asymptotic expansion are somehow absorbed in the renormalization, we arrive at

$$
\mathcal{A}(\varphi)=-2 i a_{n}\left(\gamma^{5} \varphi, D\right)
$$

that is just the expression (7.27) obtained above in the zeta function regularization. This method, based on calculations of the regularized Jacobians, can be applied to conformal anomaly as well $[222]^{29}$.

The most essential ingredient of the anomaly calculation presented in this section is the homogeneous transformation low (7.19) for the Dirac operator. This homogeneity allowed us to restore the power $-s$ in the transformation law (7.4) for the zeta function and to obtain a simple expression for the anomaly (7.26). It is clear therefore that as long as the operators transform homogeneously we shall obtain relatively simple local expressions for corresponding anomalies (understood as variations of the effective action with respect to infinitesimal transformation of the background fields). This suggests to consider extensions of the chiral group. For example, one of such extensions [346] identifies the group parameters with the diquark fields.

\subsection{Remarks on the Index Theorem}

This report is mainly devoted to local aspects of the heat kernel expansion. There is however one "global" application of the heat kernel which cannot be ignored. This is the Index Theorem. In this section we briefly sketch formal mathematical aspects of the index construction and its' relation to the heat kernel. For more details we refer to $[177,245,189,65,428]$. Physical applications of the index theorem to gravity, gauge theories and strings are so numerous that we cannot even mention them all.

The index theorem was first formulated by Atiyah and Singer [22], and the heat kernel approach appeared later in $[236,18]$. Roughly speaking, their

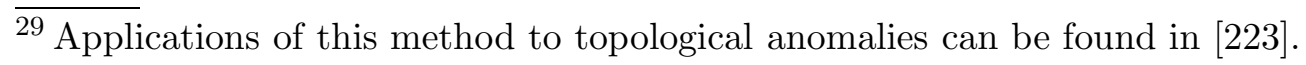


construction is as follows. Consider two vector bundles $V_{1}$ and $V_{2}$ over a manifold $M$. Let the operator $P$ map $V_{1}$ to $V_{2}$, and let $V_{1,2}$ have non-degenerate inner products $(,)_{1,2}$ so that one can define an adjoint $P^{\dagger}$ by the equation $\left(\phi_{2}, P \phi_{1}\right)_{2}=\left(P^{\dagger} \phi_{2}, \phi_{1}\right)_{1}$. As an example, one can keep in mind $V_{1}$ (respectively, $V_{2}$ ) describing positive (respectively, negative) chirality spinors. In this example $P$ is a part of the Dirac operator (see eq. (7.44) below). Let us define two operators $D_{1}=P^{\dagger} P$ and $D_{2}=P P^{\dagger}$ acting on (smooth sections of) $V_{1}$ and $V_{2}$, and let us suppose that $D_{1}$ and $D_{2}$ are elliptic. In this case we deal with an elliptic complex.

Since elliptic operators may have only a finite number of zero modes (say, $N_{1}$ and $N_{2}$ for $D_{1}$ and $D_{2}$ respectively), we may define the index by the following equations:

$$
\begin{aligned}
\operatorname{index}(P) & =N_{1}-N_{2} \\
& =\operatorname{dim} \operatorname{ker} D_{1}-\operatorname{dim} \operatorname{ker} D_{2} \\
& =\operatorname{dim} \operatorname{ker} P-\operatorname{dim} \operatorname{ker} P^{\dagger} .
\end{aligned}
$$

We also have the intertwining relations

$$
D_{1} P^{\dagger}=P^{\dagger} D_{2}, \quad P D_{1}=D_{2} P
$$

which tell us that non-zero eigenvalues of $D_{1}$ and $D_{2}$ coincide. Consider now the heat kernels,

$$
K\left(t, D_{1}\right)-K\left(t, D_{2}\right)=\sum_{\left(\lambda_{1}\right)} e^{-t \lambda_{1}}-\sum_{\left(\lambda_{2}\right)} e^{-t \lambda_{2}}=N_{1}-N_{2}=\operatorname{index}(P)
$$

(where $\lambda_{1,2}$ are eigenvalues of $D_{1,2}$ ).

To be more specific, let us suppose that $D_{1}$ and $D_{2}$ are of Laplace type and that boundary conditions and singularities (if any) are such that the powerlaw expansion (2.21) exists. Then, expanding both sides of (7.41) in a power series of $t$ one obtains:

$$
\begin{aligned}
& a_{k}\left(D_{1}\right)-a_{k}\left(D_{2}\right)=0 \quad \text { for } \quad k \neq n, \\
& a_{n}\left(D_{1}\right)-a_{n}\left(D_{2}\right)=\operatorname{index}(P) .
\end{aligned}
$$

The last equation provides a simple way to calculate the index from known heat kernel expansion. It also allows to understand a very important property of the index, namely, that it is a homotopy invariant under quite general assumptions. Indeed, suppose that $P$ depends on a parameter $\alpha$ in such a way that all geometric quantities (including the metric, connection, the matrix potential, the boundary conditions etc.) corresponding to $D_{1}$ and $D_{2}$ are smooth functions of $\alpha$. Clearly, no "essential" deformations, like changing the order of the operator, or adding higher derivative terms to the boundary conditions, or including new types of the singularities, are allowed. Under these smoothness assumptions $a_{n}\left(D_{1}\right)$ and $a_{n}\left(D_{2}\right)$ are smooth functions of $\alpha$ as well. Therefore, 
the index is also a smooth function of $\alpha$. Since the index is an integer, it can only be a constant. Hence, index $(P)$ is invariant under the deformations described above.

The relations (7.42) are also useful for calculations of the heat kernel coefficients as they give restrictions on the universal constants appearing in front of independent invariants (see, e.g., [245]).

Let us consider an example of the spin complex. Let $M$ be an even dimensional manifold admitting a spin structure (so that one can define spinor fields on $M$ ). In a suitable basis the chirality matrix may be presented in the diagonal form (at this point it may be useful to consult sec. 3.3):

$$
\gamma_{5}=\left(\begin{array}{cc}
I & 0 \\
0 & -I
\end{array}\right) .
$$

Let $V_{1}$ correspond the +1 eigenvalue of $\gamma_{5}$ (positive chirality), and $V_{2}-$ to -1 . The Dirac operator (3.26) anti-commutes with $\gamma_{5}$ and, therefore, may be represented in the form:

$$
\not D=\left(\begin{array}{cc}
0 & P^{\dagger} \\
P & 0
\end{array}\right)
$$

We also have:

$$
\not D^{2}=\left(\begin{array}{cc}
P^{\dagger} P & 0 \\
0 & P P^{\dagger}
\end{array}\right) .
$$

We conclude that index $(P)$, which now measures difference between dimensions of the null subspaces of $\not D$ with positive and negative chirality, is equal to global chiral anomaly:

$$
\operatorname{index}(P)=\operatorname{Tr}\left(e^{-t P^{\dagger} P}\right)-\operatorname{Tr}\left(e^{-t P P^{\dagger}}\right)=\operatorname{Tr}\left(\gamma_{5} e^{-t \not D^{2}}\right)=a_{n}\left(\gamma_{5}, \not D^{2}\right)
$$

Therefore, the index can be calculated by the methods of the previous section. In particular, the index of the Dirac operator in two and four dimensions on flat manifolds without background axial vector fields $\left(A_{\mu}^{5}=0\right)$ can be read off from (7.31) and (7.32) with $\varphi:=i / 2$. We also see that chiral anomaly provides very important information on topology of the background.

One can associate an index with any elliptic complex which must not necessarily contain only two vector bundles. In general, one has a sequence of bundles $V_{p}$ and a family of operators $P_{p}$ which map (smooth sections of) $V_{p}$ to (smooth sections of) $V_{p+1}$. One requires that $P_{p} P_{p+1}=0$ and that the corresponding "Laplacians" $D_{p}=P_{p}^{\dagger} P_{p}+P_{p-1} P_{p-1}^{\dagger}$ are elliptic. Important examples include the de Rham complex (for which $V_{p}$ consists of $p$-forms on $M$ and $P$ is the exterior derivative), the Dolbeaux complex which deals with the forms on a complex manifold, and the signature complex which treats selfdual and anti-selfdual forms. More details can be found in [177]. Another very 
important construction of this type is the Witten index [422] which constraints the supersymmetry breaking. 


\section{Resummation of the heat kernel expansion}

The heat kernel coefficients define the one-loop counterterms in the background field formalism. In many cases the heat kernel can also give a useful information on the finite part of the effective action. Just one of the examples is the large mass expansion (1.22) which is valid when all background fields and their derivatives are small compared to the mass of the quantum field. In order to get the effective action in other limiting cases one has to re-arrange the heat kernel expansion ${ }^{30}$.

\subsection{Modified large mass expansion}

In many physical applications there is a quantity $\mathcal{M}^{2}$ which is large compared to the rest of the background fields and their derivatives. Therefore, it is a well motivated problem to construct an expansion of the effective action in a power series of $\mathcal{M}^{-1}$. To do so, one has to re-express the heat kernel as

$$
K(t ; D)=e^{-t \mathcal{M}^{2}} \sum_{k} t^{(k-n) / 2} \tilde{a}_{k} .
$$

To obtain the effective action one has to integrate the heat kernel over $t$ (cf eq. (1.18)). To simplify the argumentation we assume the cut-off regularization (1.20), although in other regularization schemes the result will be essentially the same. The divergent and finite parts of the effective action are given by (1.21) and (1.22) respectively up to the obvious replacements: $m \rightarrow \mathcal{M}$, $(4 \pi)^{-n / 2} b_{k} \rightarrow \tilde{a}_{k}$. After a suitable renormalization, this procedure indeed gives a large $\mathcal{M}$ expansion of the effective action.

Therefore, the problem is reduced to calculation of $\tilde{a}_{k}$. If $\mathcal{M}$ commutes with $D$ the coefficient $\tilde{a}_{k}$ are the heat kernel coefficients for the operator $D-\mathcal{M}^{2}$. This case returns us to the standard large mass expansion. If $\mathcal{M}^{2}$ does not commute with $D$ then

$$
e^{-t D} \neq e^{-t\left(D-\mathcal{M}^{2}\right)} e^{-t \mathcal{M}^{2}} .
$$

To achieve an equality the right hand side of (8.2) must be corrected by commutator terms. In this case calculation of $\tilde{a}_{k}$ requires some amounts of extra work.

Heavy particles of non-equal masses described by the mass matrix $\mathcal{M}^{2}$ is probably the most immediate example of a physical system to which the modified large mass expansion should be applied. Corresponding technical tools were developed recently $[355,354,353]$.

The next example is a scalar field in curved space (cf. sec 3.1). Parker and Toms suggested $[359,358]$ to use the modified large mass expansion with

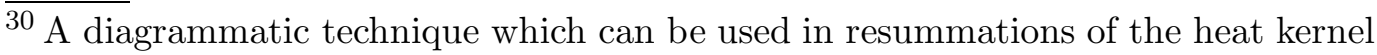
expansion is described in [331].
} 
$\mathcal{M}^{2}=m^{2}+\xi R$ which partially sums up contributions of the scalar curvature $R$ to the effective action. This formalism was developed further in $[284,393]$.

In some cases the term-by-term integration of the heat kernel expansion gives good estimates of the vacuum energy even if no large parameter is explicitly present in the model (see, e.g. calculations of quantum corrections to the mass of two dimensional solitons $[176,9]$ ).

\subsection{Covariant perturbation theory}

Suppose that the matrix potential $E$, the Riemann curvature, and the field strength $\Omega$ are small but rapidly varying. Can we get any information on the heat kernel coefficients containing a fixed power of the quantities listed above and arbitrary number of derivatives? For the linear and quadratic orders on a manifold without boundaries the answer is positive and the results can be obtained either by the functorial methods of sec. $4.1[239,92]$ or by solving the DeWitt equation $[24,25,26]$ (cf. sec. 4.3). Retaining only the leading terms we have for $k \geq 1$ :

$$
\begin{gathered}
a_{2 k}(x, D)=(4 \pi)^{-n / 2}\left[\alpha_{1}(k) \Delta^{k-1} R+\alpha_{2}(k) \Delta^{k-1} E\right. \\
+ \text { higher order terms }]
\end{gathered}
$$

where $[237,239]$

$$
\alpha_{1}(k)=\frac{k ! k}{(2 k+1) !}, \quad \alpha_{2}(k)=\frac{2 k !}{(2 k) !} .
$$

One can sum up the expansion (8.3) and corresponding higher order terms to obtain an information on the behaviour of the full heat kernel in the limit described above. There is, however, a more straightforward method to control non-localities which is called the covariant perturbation theory $[56,57,58]$. To make the idea of this method most transparent we consider a simple example of flat $M$ and zero connection $\omega$. The exponent $\exp (-t D)=\exp (t(\Delta+E))$ with $\Delta=\partial_{\mu}^{2}$ can be expanded in a power series in $E$ :

$$
e^{-t D}=e^{t \Delta}+\int_{0}^{t} d s e^{(t-s) \Delta} E e^{s \Delta}+\int_{0}^{t} d s_{2} \int_{0}^{s_{2}} d s_{1} e^{\left(t-s_{2}\right) \Delta} E e^{\left(s_{2}-s_{1}\right) \Delta} E e^{s_{1} \Delta}+\ldots
$$

The heat trace can be also expanded,

$$
K(t, D)=\operatorname{Tr}\left(e^{-t D}\right)=\sum_{j=0}^{\infty} K_{j}(t),
$$

where $K_{j}$ contains the $j$ th power of $E$.

Covariant perturbation theory approach prescribes to take the 0th order 
heat kernel in the free space form (cf. eq. (1.12) for $m=0$ ):

$$
K_{0}(x, y ; t)=(4 \pi t)^{-n / 2} \exp \left(-\frac{(x-y)^{2}}{4 t}\right)
$$

which is an exact kernel on $M=\mathbb{R}^{n}$ only $^{31}$. This formula neglects all global contributions and, therefore, is valid only for sufficiently close $x$ and $y$ and for small $t$. We have:

$$
K_{0}(t)=\int_{M} d^{n} x \sqrt{g} \operatorname{tr}_{V} K_{0}(x, x ; t)=(4 \pi t)^{-n / 2} \int_{M} d^{n} x \sqrt{g} \operatorname{tr}_{V}\left(I_{V}\right),
$$

where $\operatorname{tr}_{V} I_{V}$ simply counts discrete (spin and internal) indices of $D$. This formula reproduces the $a_{0}$ contribution to the heat kernel.

In the next order, we have:

$$
\begin{aligned}
K_{1}(t) & =\operatorname{Tr}\left(\int_{0}^{t} d s e^{(t-s) \Delta} E e^{s \Delta}\right)=\operatorname{Tr}\left(\int_{0}^{t} d s e^{t \Delta} E\right) \\
& =t \operatorname{tr}_{V} \int_{M} d^{n} x \sqrt{g} K_{0}(x, x ; t) E(x) \\
& =\frac{t}{(4 \pi t)^{n / 2}} \int_{M} d^{n} x \sqrt{g} \operatorname{tr}_{V} E(x),
\end{aligned}
$$

where we used the cyclic property of the trace and the expression (8.7) for the 0th order heat kernel. This expression is consistent with the $k=1$ term of (8.3). The terms with $k>1$ are total derivatives, and, therefore, they do not contribute to the integrated heat kernel.

The quadratic order of the heat kernel reads

$$
\begin{aligned}
K_{2}(t)=\operatorname{Tr}\left(\int_{0}^{t} d s_{2} \int_{0}^{s_{2}} d s_{1} e^{\left(t-s_{2}\right) \Delta} E e^{\left(s_{2}-s_{1}\right) \Delta} E e^{s_{1} \Delta}\right) \\
=\operatorname{tr}_{V} \int_{M} d y \int_{M} d z \int_{0}^{t} d s_{2} \int_{0}^{s_{2}} d s_{1} K_{0}\left(z, y ; t-s_{2}+s_{1}\right) E(y) \\
\times K_{0}\left(y, z ; s_{2}-s_{1}\right) E(z) .
\end{aligned}
$$

Next we introduce the rescaled variables $\xi=s / t$ and get rid of redundant integrations to obtain:

$$
\begin{aligned}
& K_{2}(t)=\frac{t^{2}}{2} \operatorname{tr}_{V} \int_{M} d y \int_{M} d z \int_{0}^{1} d \xi K_{0}(z, y ; t(1-\xi)) E(z) \\
& \times K_{0}(y, z ; t \xi) E(z)
\end{aligned}
$$

$\overline{31}$ The original paper [56] contained a curved space generalisation of (8.7). This, however, does not improve the global issues discussed below. 
Now we use the identity

$$
K_{0}(z, y ; t(1-\xi)) K_{0}(y, z ; t \xi)=(4 \pi t)^{-n / 2} K_{0}(z, y ; t \xi(1-\xi))
$$

and relate the heat kernel on the right hand side of (8.12) to a matrix element of $\exp (t \xi(1-\xi) \Delta)$ to obtain the final result:

$$
K_{2}(t)=\frac{t^{2}}{(4 \pi t)^{n / 2}} \int_{M} d^{n} x \sqrt{g} E f(-t \Delta) E,
$$

where the non-local form-factor $f$ reads:

$$
f(q)=\frac{1}{2} \int_{0}^{1} d \xi e^{-q \xi(1-\xi)}=\frac{1}{2} e^{-q / 4} \sqrt{\pi / q} \operatorname{Erfi}[\sqrt{q} / 2] .
$$

As we have already discussed above, applicability of this formula is limited by our choice of $K_{0}(x, y ; t)$. Namely, the potential $E(x)$ should have a compact support and $t$ should be reasonably small. Note, that the small $t$ approximation in the expansion (8.5) is self-consistent: if $t$ is small, the integration variables $s_{i}$ are even smaller.

The main difficulty in constructing an expansion in powers the Riemann curvature and of the field strength is to organise the procedure in a covariant way. The details of the construction and higher order form-factors can be found in $[56,57,58,50,49]$. From further developments of this method we mention the work of Gusev and Zelnikov [267] who demonstrated that in two dimension one can achieve considerable simplifications in the perturbation expansion by using the dilaton parametrisation of the potential (cf. eq. (7.15)). Recently Barvinsky and Mukhanov [53] suggested a new method for calculation of the non-local part of the effective action based on the resummation of the perturbation series for the heat kernel. This method was extended in [48] to include late time asymptotics of the heat kernel in curved space.

Let us stress that the expansion (8.5) can be used also for singular potentials. For example, it is very effective for the calculation of the heat kernel for $\delta$-potentials concentrated on a co-dimension one subsurface $[231,82,330]$ (cf. sec. 6.3). If the $\delta$-potential has its support on a submanifold of co-dimension greater than one, the expansion diverges [82]. With a suitable choice of the zeroth order heat kernel $K_{0}(x, y ; t)$ and of an operator to replace $E$ in (8.5) one can treat manifolds with boundaries [74] where the perturbative expansion takes the form of the multiple reflection expansion $[42,43,272]$.

\section{3 "Low energy" expansion}

Let us now turn to the opposite case when the derivatives are less important when the potential and the curvatures. In this case one has to collect the terms which are of a fixed order in the derivatives, but contain all powers of $E, R$ and $\Omega$. Since the derivatives are sometimes identified with the energy, 
this approximation is being called the low energy expansion. This scheme goes back to Schwinger's calculations [379] in constant electromagnetic fields ${ }^{32}$.

Let us consider a simple example [71]. Let $M=\mathbb{R}^{2}$. Consider a scalar particle in constant electromagnetic field with the field strength $F_{12}=-F_{21}=$ $B$. As a potential we choose $A_{1}=0, A_{2}=B x^{1}$. Then the operator acting on quantum fluctuations is

$$
D=-\partial_{1}^{2}-\left(\partial_{2}-i B x^{1}\right)^{2}+m^{2}
$$

This operator commutes with $\partial_{2}$. Therefore, we can look for the eigenfunctions of $D$ in the form $\phi_{k}(x)=\exp \left(i k x^{2}\right) \tilde{\phi}\left(x^{1}\right)$.

$$
D \phi_{k}(x)=\left(-\partial_{1}^{2}+B^{2}\left(x^{1}-k / B\right)^{2}+m^{2}\right) \phi_{k}(x) .
$$

In the $x^{1}$ direction we have the one-dimensional harmonic oscillator potential (cf. (6.3)). Therefore, the eigenvalues are $\lambda_{k, p}=(2 p+1)|B|+m^{2}$. These eigenvalues do not depend on $k$. For this reason the heat kernel

$$
K(t, D)=\sum_{k, p} \exp \left(-t \lambda_{k, p}\right)
$$

is ill defined. To overcome this difficulty it was suggested [71] to put the system in a box (without specifying any boundary conditions, however) and replace the sum over $k$ by the degeneracy factor $(|B| \mathrm{Vol}) /(2 \pi)$, where $\mathrm{Vol}$ is volume of the box. The degeneracy factor is chosen in such a way that the resulting heat kernel

$$
K(t, D)=\frac{B V \mathrm{ol}}{4 \pi} e^{-m^{2} t}[\sinh (B t)]^{-1}
$$

behaves as $\mathrm{Vol} /(4 \pi t)$ in the limit $B \rightarrow 0$.

Calculations of the effective action in covariantly constant background gauge fields by the spectral theory methods have been performed by many authors $[59,151,185,183,71]$. Such calculations were motivated, at least partially, by various models of quark confinement (see [356,4] and references therein).

There exists an algebraic method $[27,29]$ which allows to evaluate the low energy heat kernel by using exclusively the commutator algebra. Let us briefly formulate the results of $[27,29]$. Consider a flat manifold $\left(R_{\mu \nu \rho \sigma}=0\right)$ with the background fields satisfying the "low-energy conditions":

$$
\nabla_{\mu} \Omega_{\mu \nu}=0, \quad \nabla_{\mu} \nabla_{\nu} \nabla_{\rho} E=0
$$

with usual definitions of $E$ and $\Omega$ (see (2.1) - (2.10)). Moreover, let us suppose that the background is "approximately abelian", i.e. that $\Omega_{\mu \nu}, E$ and all their covariant derivatives are mutually commuting matrices.

\footnotetext{
${ }^{32}$ We like to mention also the paper [275] which treated the effective action in external electromagnetic field from a different point view.
} 
With all these assumptions a closed expression for the heat kernel may be obtained:

$$
K(t ; x, x ; D)=(4 \pi t)^{-n / 2} \exp \left(t E+\Phi(t)+\frac{t^{3}}{4} E_{; \mu} \Psi^{\mu \nu} E_{; \nu}\right)
$$

where $\Phi$ and $\Psi$ are complicated functions of $t, E$ and $\Omega$ [29]. If $\nabla_{\mu} \nabla_{\nu} E=0$,

$$
\begin{aligned}
& \Phi(t)=-\frac{1}{2} \ln \operatorname{det}\left(\frac{\sinh t \Omega}{t \Omega}\right), \\
& \Psi(t)=(t \Omega)^{-2}(t \Omega \operatorname{coth}(t \Omega)-1),
\end{aligned}
$$

where $\Omega$ has to be understood as a space-time matrix $\Omega_{\mu \nu}$, so that multiplication in (8.21) is the matrix multiplication, and det is the determinant of an $n \times n$ matrix. These formulae generalise the equation (8.18) and justify the choice of the degeneracy factor made to derive it.

If $\Omega=0$,

$$
\begin{aligned}
& \Phi(t)=-\frac{1}{2} \ln \operatorname{det}\left(\frac{\sinh (2 t \sqrt{P}}{2 t \sqrt{P}}\right), \\
& \left.\Psi(t)=-(t \sqrt{P})^{-3}(\tanh (t \sqrt{P})-t \sqrt{P})\right),
\end{aligned}
$$

where $P_{\mu \nu}=-(1 / 2) \nabla_{\mu} \nabla_{\nu} E$. In the particular case of one-dimensional harmonic oscillator these formulae reproduce (6.5).

In curved space the best one can do in the framework of the low-energy expansion is to consider locally symmetric manifolds, i.e. vanishing $\Omega$, covariantly constant Riemann curvature and constant $E$. In this case formulae similar to the ones presented above are available $[28,30]$. Covariantly constant curvature means that locally the manifold $M$ is a symmetric space. Various approaches to the heat kernel on such manifolds are described in detail in monographs and survey articles $[282,115,109]$. In particular, very detailed in-

formation may be obtained for group manifolds (see, for example, [153]) and for hyperbolic spaces [110].

\subsection{Heat kernel on homogeneous spaces}

In this section we briefly explain how one can find the spectrum of some "natural" differential operators on homogeneous spaces by purely algebraic methods. We start with some basic facts from differential geometry and harmonic analysis $[301,276]$. Consider a homogeneous space $G / H$ of two compact finite-dimensional Lie groups $G$ and $H$. The Lie algebra $\mathcal{G}$ of $G$ can be decomposed as

$$
\mathcal{G}=\mathcal{H} \oplus \mathcal{M}
$$


where $\mathcal{H}$ is the Lie algebra of $H$ and $\mathcal{M}$ is the complement of $\mathcal{H}$ in $\mathcal{G}$ with respect to some bi-invariant metric. We have:

$$
[\mathcal{H}, \mathcal{M}] \subset \mathcal{M}
$$

where [, ] is the Lie bracket on $\mathcal{G}$. If, moreover, $[\mathcal{M}, \mathcal{M}] \subset \mathcal{H}$, then $G / H$ is a symmetric space. We do not impose this restriction.

$\mathcal{M}$ can be identified with the tangent space to $M=G / H$ at the origin (i.e. at the point which represents the unit element of $G$ ). Eq. (8.24) tells us that $\mathcal{H}$ acts on $\mathcal{M}$ by some (orthogonal) representation. This action defines the embedding

$$
\mathcal{H} \subset \operatorname{so}(n) .
$$

All physical fields are classified according to certain representations of the Lie algebra $s o(n)$. Restrictions of these representations to $\mathcal{H}$ define transformation properties of the field with respect to $\mathcal{H}$. From now on we work with each irreducible representation of $\mathcal{H}$ separately.

The field $\Phi^{A}$ belonging to an irreducible representation $\mathcal{T}(H)$ can be expanded as (see, e.g., [373]):

$$
\Phi_{A}(x)=(\mathrm{Vol})^{-\frac{1}{2}} \sum_{j, \xi, q} \sqrt{\frac{d_{j}}{d_{\mathcal{T}}}} \mathcal{T}_{A \xi, q}^{(j)}\left(g_{x}^{-1}\right) \phi_{q, \xi}^{(j)},
$$

where Vol is the volume of $G / H, d_{\mathcal{T}}=\operatorname{dim} \mathcal{T}(H)$. We sum over the representations $\mathcal{T}^{(j)}$ of $G$ which give $\mathcal{T}(H)$ after reduction to $H$. $\xi$ labels multiple components $\mathcal{T}(H)$ in the branching $\mathcal{T}^{(j)} \downarrow H, d_{j}=\operatorname{dim} \mathcal{T}^{(j)}$.q runs from 1 through $d_{j}$. The matrix elements of $\mathcal{T}^{(j)}$ have the following orthogonality property

$$
\int_{M} d^{n} x \sqrt{g} \mathcal{T}_{A \zeta, q}^{(j) \dagger}\left(g_{x}^{-1}\right) \mathcal{T}_{A \xi, p}^{\left(j^{\prime}\right)}\left(g_{x}^{-1}\right)=(\mathrm{Vol}) d_{j}^{-1} d_{\mathcal{T}} \delta_{\zeta \xi} \delta_{p q} \delta_{j j^{\prime}}
$$

Therefore, to construct the harmonic expansion on $G / H$ it is necessary to have powerful methods for reduction of the representations from $G$ to $H$. There are several standard [46,387] and less standard [311,310] techniques which may be used depending on the particular homogeneous space.

It is important that not only the harmonic expansion but also the spectrum of the invariant operators can be analysed by the group theoretical methods. The covariant derivative on $G / H$ reads [301]:

$$
\nabla_{\mu}=\nabla_{\mu}^{[c]}+\Gamma_{\mu}^{[R]}
$$

Here $\nabla^{[c]}$ is the canonical covariant derivative on $G / H$. At the origin $\nabla^{[c]}$ can be identified with the tangent space generators from $\mathcal{M}$ taken in the representation $\mathcal{T}^{(j)}$. The part $\Gamma_{\mu}^{[R]}$ depends on the invariant metric on $G / H$ and on the structure constants of $\mathcal{G}$ restricted to $\mathcal{M}$. On symmetric spaces such structure constants are zero and, therefore, the Laplace-Beltrami operator has 
a particularly simple form:

$$
D \simeq \nabla^{\mu[c]} \nabla_{\mu}^{[c]} \simeq C_{2}(G)-C_{2}(H)
$$

where $C_{2}$ are quadratic Casimir operators of $G$ and $H$ which depend on the representations $\mathcal{T}^{(i)}$ and $\mathcal{T}(H)$ respectively. On general homogeneous spaces the expressions are a bit more complicated (see, e.g., [407,310] for explicit examples). In any case, eigenvalues of $D$ are given by a second order polynomial $Q\left(m_{1}, \ldots, m_{k}\right)$ of several natural numbers $m_{l}$. These eigenvalues are in general degenerate with multiplicities defined essentially by dimensions of the representations of $G$ and $H$. They are also polynomials ${ }^{33}$ in $m_{l}$. The heat kernel is then represented as an infinite sum

$$
K(t, D)=\sum_{m_{l}} N\left(m_{1}, \ldots, m_{k}\right) \exp \left(-t Q\left(m_{1}, \ldots, m_{k}\right)\right)
$$

There exist several tricks which can be used to evaluate the $t \rightarrow 0$ asymptotics of such sums. For example, one may use the Poisson summation formula (4.4). By taking derivatives with respect to $t$ one obtains

$$
\sum_{l \in \mathbb{Z}} l^{2 r} \exp \left(-t l^{2}\right) \simeq \sqrt{\pi} 2^{-r}(2 r-1) ! ! t^{-(2 r+1) / 2}+\mathcal{O}\left(e^{-1 / t}\right)
$$

for $r \in \mathbb{N}$.

More general polynomials of $l$ may be treated by the Euler-Maclaurin formula which reduces sums to the integrals. Let $F(\tau)$ be a function defined on $0 \leq \tau<\infty$. If the $2 m$-th derivative $F^{(2 m)}(\tau)$ is absolutely integrable on $(0, \infty)$

$$
\begin{aligned}
\sum_{i=0}^{k} F(i)-\int_{0}^{\infty} & F(\tau) d \tau=\frac{1}{2}[F(0)+F(k)] \\
& +\sum_{s=1}^{m-1} \frac{B_{2 s}}{(2 s) !}\left[F^{(2 s-1)}(k)-F^{(2 s-1)}(0)\right]+\operatorname{Rem}_{m}(n),
\end{aligned}
$$

where the reminder $\operatorname{Rem}_{m}$ satisfies:

$$
\left|\operatorname{Rem}_{m}(n)\right| \leq\left(2-2^{1-2 m}\right) \frac{\left|B_{2 m}\right|}{(2 m) !} \int_{0}^{n}\left|F^{(2 m)}(\tau)\right| d \tau
$$

$B_{s}$ are the Bernoulli numbers. If $F(i)$ is taken to be $N_{i} \exp \left(-t \lambda_{i}\right)$, one can take limit $k \rightarrow \infty$ in (8.32) and restrict the summation to some finite $m$ to obtain asymptotic series for the heat kernel. For example, one can easily recover the expansion (8.31).

$\overline{33}$ The spectrum of differential operators on coset spaces can be calculated exactly even if one adds a homogeneous gauge field (see [152] and references therein). 
In many cases calculation can be done by means of the Mellin transform. This method will be clear from the following example. Consider

$$
K(t)=\sum_{k=1}^{\infty} k \exp \left(-t k^{2}\right)
$$

The Mellin transform of $K(t)$ is

$$
\tilde{K}(s)=\sum_{k} k \int_{0}^{\infty} d x x^{s-1} \exp \left(-x k^{2}\right)=\sum_{k=1}^{\infty} k^{1-2 s} \Gamma(s)=\zeta_{R}(2 s-1) \Gamma(s),
$$

where $\zeta_{R}$ is the Riemann zeta-function. Inverse Mellin transformation gives

$$
K(t)=\frac{1}{2 \pi i} \int_{C} t^{-s} \zeta_{R}(2 s-1) \Gamma(s) d s
$$

The contour $C$ covers all poles of the integrand at $s=1,0,-1,-2, \ldots$ By calculating the residues we obtain the desired expansion:

$$
K(t)=\frac{1}{2 t}-\frac{1}{12}-\frac{t}{120}-\frac{t^{2}}{504}-\frac{t^{3}}{1440}+O\left(t^{4}\right)
$$

Actually, the techniques introduced above should only be used for complicated multi-parameter sums (see, e.g., [310]). For a simple one-parameter sum it is easier to use combinations of known asymptotic series (cf. Appendix of Ref. [67]).

In this method complexity of calculations of the heat kernel coefficients $a_{k}$ is almost independent of $k$. Therefore, the algebraic techniques were applied to higher dimensional theories where one needs higher heat kernel coefficients to perform renormalization or to calculate the anomalies. Rather naturally, the most simple toroidal spaces were considered first [15, 16,372,302,210,102], and then computations on spheres were performed [117, 295, 290, 67, 68, 249, 105, $106,180]$. Other homogeneous spaces were considered for example in $[310,408]$. In the same approach non-minimal operators on homogeneous spaces were treated in $[401,403]$. We refer to [115] for a more extensive literature survey. 


\section{Exact results for the effective action}

In the previous section we have seen that the heat kernel can be calculated exactly for all values of $t$ if the background satisfies certain symmetry or smoothness conditions. In this section it will be demonstrated that for some classes of the operators the effective action can be calculated exactly without imposing any restrictions on behaviour of the background fields. These are the cases when the variation of $\zeta^{\prime}(0)$ with respect to the background can be reduced to the heat kernel coefficients which are locally computable. Then the variation is integrated to the effective action.

The most immediate example of the variations admissible for this scheme is quantum anomalies (cf. sec. 7). Historically, the first action obtained by integration of an anomaly was the chiral Wess-Zumino action. We do not consider this action here since such a consideration would require a lot of additional technical devices. The reader can consult excellent original papers $[417,424]^{34}$. The next subsection is devoted to another well know example, which is the Polyakov action obtained through integration of the conformal anomaly in two dimensions.

In sec. 9.2 below we consider a more complicated situation when relevant variation of the effective action leads to a linear combination of the heat kernel coefficients of several different operators. Typically in this case one obtains exact relations between the effective actions rather than the effective actions themselves.

\subsection{The Polyakov action}

Let us consider a two-dimensional Riemannian manifold $M$ without boundary and a scalar field $\phi$ minimally coupled to the geometry. This means that we choose $\Phi=0$ in the action (7.9) and $\Psi=0$ in the inner product (7.10). In two dimensions any metric is conformally flat, i.e. by a suitable choice of the coordinates one may transform it to the conformal gauge

$$
g_{\mu \nu}=e^{2 \rho(x)} \eta_{\mu \nu}, \quad \eta_{\mu \nu}=\operatorname{diag}(1,1)
$$

In this gauge

$$
\sqrt{g} R=-2 \eta^{\mu \nu} \partial_{\mu} \partial_{\nu} \rho .
$$

The effective action $W$ depends on $\rho$ only (since there is no other external field in this problem). We substitute (7.16) with $\Phi=\Psi=0$ in (7.3) to obtain

$$
\delta W=\frac{1}{12 \pi} \int_{M} d^{2} x(\delta \rho) \eta^{\mu \nu} \partial_{\mu} \partial_{\nu} \rho
$$

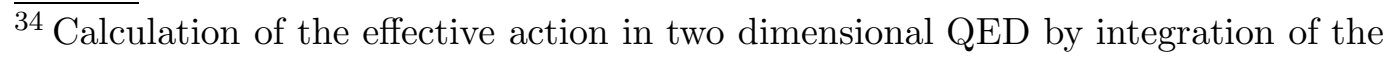
anomaly may be found in [279].
} 
This equation can be integrated with the initial condition $W(\rho=0)=0$.

$$
W=\frac{1}{24 \pi} \int_{M} d^{2} x \rho \eta^{\mu \nu} \partial_{\mu} \partial_{\nu} \rho
$$

By returning to the covariant notations we obtain the famous Polyakov action [360] (see also [309]):

$$
W=\frac{1}{96 \pi} \int_{M} d^{2} x \sqrt{g} R \frac{1}{\Delta} R
$$

Note, that on a compact manifold one has to take into account contributions from the zero modes which lead to an additional term in the Polyakov action [157].

The problem of finding an action whose conformal variation reproduces the conformal anomaly can be posed in higher dimension as well [144] and some physical consequences of such actions can be studied [218,219]. Complete expressions for the conformal anomaly induced effective action in four dimensions were obtained in $[366,100]$. Conformal action in the presence of boundaries was constructed in [170]. One should keep in mind that unless the background is conformally flat the conformal action represents only a part of the full effective action. Applicability and limitations of the conformal action approach are discussed in [188,41,267].

\subsection{Duality symmetry of the effective action}

In this section we deal with variations of the effective action which can be expressed through zeta functions of several operators (in contrast to genuine anomalous transformations of sec. 7 which involve a single zeta function each).

A rather simple example can be found in the paper [304] which considered two operators of Dirac type:

$$
\not D=i \gamma^{\mu} e^{\Psi} \partial_{\mu} e^{-\Phi}, \quad \not D^{\dagger}=i \gamma^{\mu} e^{-\Phi} \partial_{\mu} e^{\Psi}
$$

on flat two-dimensional manifold without boundary depending on two scalar functions $\Phi$ and $\Psi$. Let us now consider small variations of $\Phi$ and $\Psi$. According to (7.4) the zeta function of corresponding Laplacian changes as

$$
\delta \zeta\left(s, \not D \not D^{\dagger}\right)=-2 s \operatorname{Tr}\left[\left(\not D \not D^{\dagger}\right)^{-s} \delta \Psi-\left(\not D^{\dagger} \not D\right)^{-s} \delta \Phi\right]
$$

Variation of $\zeta^{\prime}(0)$ reads:

$$
\begin{aligned}
\delta \zeta^{\prime}\left(0, \not D \not D^{\dagger}\right) & =-2\left[\zeta\left(0, \delta \Psi, \not D \not D^{\dagger}\right)-\zeta\left(0, \delta \Phi, \not D^{\dagger} \not D\right)\right] \\
& =-2\left[a_{2}\left(\delta \Psi, \not D \not D^{\dagger}\right)-a_{2}\left(\delta \Phi, \not D^{\dagger} \not D\right)\right] .
\end{aligned}
$$

Here we used that $\not D \not D^{\dagger}$ and $\not D^{\dagger} \not D$ are operators of Laplace type, and, therefore, 
the corresponding zeta functions are regular at $s=0$. In the last line we replaced $\zeta(0)$ by the heat kernel coefficients by means of (2.27).

Next we have to rewrite $\not D \not D^{\dagger}$ and $\not D^{\dagger} \not D$ in the canonical form (2.2) with the help of (2.3), (2.4). For $\not D \not D^{\dagger}$ we have:

$$
\begin{aligned}
& g^{\mu \nu}=e^{2(\Psi-\Phi)}, \quad \omega_{\mu}=\Psi_{, \mu}-\gamma^{\nu} \gamma_{\mu} \Phi_{, \nu}, \\
& E=\Phi_{, \mu}{ }^{\mu}, \quad R=-2(\Phi-\Psi)_{, \mu}{ }^{\mu},
\end{aligned}
$$

where comma denotes ordinary partial derivatives, all contractions are performed with the effective metric $g^{\mu \nu}$. Note, that in two dimensions $\Gamma_{\mu \nu}^{\rho} g^{\mu \nu}=0$ in the conformal coordinates. To obtain relevant geometric quantities for $\not D^{\dagger} \not D$ one has to replace $\Phi$ by $-\Psi$ and vice versa. Equation (4.27) gives:

$$
\delta \zeta^{\prime}\left(0, \not D \not D^{\dagger}\right)=-\frac{1}{6 \pi} \int d^{2} x[\delta \Psi(\Delta \Psi+2 \Delta \Phi)+\delta \Phi(\Delta \Phi+2 \Delta \Psi)]
$$

where $\Delta$ is the flat space Laplacian. Let us recall, that $\zeta^{\prime}(0, D)$ defines the functional determinant of $D$ (see eq. (2.33)). The variation $(9.10)$ can be integrated to give ${ }^{35}$

$$
\ln \operatorname{det}\left(\not D \not D^{\dagger}\right)=-\zeta^{\prime}\left(0, \not D \not D^{\dagger}\right)=\frac{1}{12 \pi} \int d^{2} x(\Psi \Delta \Psi+4 \Psi \Delta \Phi+\Phi \Delta \Phi) .
$$

We see from (9.11) that $\ln \operatorname{det}\left(\not D \not D^{\dagger}\right)=\ln \operatorname{det}\left(\not D^{\dagger} \not D\right)$ which is obviously true up to possible topological contributions from zero modes which have been neglected in this calculation. The result (9.11) was confirmed in [267] by the methods of covariant perturbation theory.

This example is clearly of (at least an academic) interest since it adds up to very few cases discussed above in this section when a closed analytic expression for the determinant may be obtained without any assumptions on smoothness of the background fields. The operator $\not D \not D^{\dagger}$ shares some similarities with the kinetic operator for non-minimally coupled scalars in two dimensions (7.12). There are good grounds to believe [305] the functional determinant (9.11) may describe the spherically symmetric part of the Hawking flux in four dimensions.

Let us consider the dilatonic operator (7.12). We take $\Psi=\Phi$ for simplicity $^{36}$. We shall be interested in properties of $\operatorname{det} D$ under reflection of the dilaton, $\Phi \rightarrow-\Phi$. The quantity $\ln \operatorname{det} D(\Phi)-\ln \operatorname{det} D(-\Phi)$ defines the dilaton shift under the $T$-duality transformations in string theory. In the string theory context this problem was solved in [378] basing on earlier results of [377]. Here we follow presentation of the paper [409].

$\overline{35}$ The heat kernel coefficients $a_{2}\left(x, \not D \not D^{\dagger}\right)$ and $a_{2}\left(x, \not D^{\dagger} \not D\right)$ are total derivatives. Therefore, the second term on the right hand side of (2.33) does not contribute.

${ }^{36}$ Variation of $\operatorname{det} D$ with respect to $\Psi$ may be expressed through the scale anomaly. Therefore, it is not a problem to find exact dependence of $\operatorname{det} D$ on $\Psi$ by using the methods of sec. 7 . 
Let us introduce two first order differential operators

$$
L_{\mu}=e^{-\Phi} \partial_{\mu} e^{\Phi}, \quad L_{\mu}^{\dagger}=-e^{\Phi} \partial_{\mu} e^{-\Phi}
$$

and associated second order operators

$$
D_{+}=L_{\mu}^{\dagger} L_{\mu}, \quad D_{-}=L_{\mu} L_{\mu}^{\dagger} .
$$

We restrict ourselves to flat two-dimensional manifolds. Therefore, position of the indices (up or down) plays no role.

We calculate again variation of the $\zeta$ function with respect to variation of $\Phi$ :

$$
\delta \zeta\left(s, D_{+}\right)=-2 s \operatorname{Tr}\left[(\delta \Phi)\left(L_{\mu}^{\dagger} L_{\mu} D_{+}^{-s-1}-L_{\mu} D_{+}^{-s-1} L_{\mu}^{\dagger}\right)\right] .
$$

The operators in the first term here recombine in $D_{+}^{-s}$. The situation with the second term is more subtle. Strictly speaking, to treat this term one has to perform the Mellin transform and use analytic continuation in $s$ (see footnote 26 in sec. 7.1). However, the result of such manipulations is almost obvious:

$$
\operatorname{Tr}\left[(\delta \Phi) L_{\mu} D_{+}^{-s-1} L_{\mu}^{\dagger}\right]=\operatorname{Tr}\left[(\delta \Phi) H^{-s}\right]
$$

where

$$
H_{\mu \nu}=L_{\mu} L_{\nu}^{\dagger}
$$

is a matrix operator acting on the space $V_{+}$of the vector fields which can be represented as $v_{\nu}=L_{\nu} f$ with some scalar function $f$. The operator (9.16) is not of Laplace type as it contains a complicated differential projector on $V_{+}$. Therefore, even though the variation (9.14) looks very similar to (9.7), for example, it cannot be used directly to evaluate $\zeta^{\prime}\left(0, D_{+}\right)$since we cannot even guarantee that (9.15) is regular at $s=0$ (in fact, it is not regular, see [409]).

We also have:

$$
\delta \zeta\left(s, D_{-}\right)=2 s \operatorname{Tr}\left[(\delta \Phi)\left(D_{-}^{-s}-\bar{H}^{-s}\right)\right]
$$

where the operator

$$
\bar{H}_{\mu \nu}=\epsilon_{\mu \mu^{\prime}} \epsilon_{\nu \nu^{\prime}} L_{\mu^{\prime}}^{\dagger} L_{\nu^{\prime}}
$$

acts on the space $V_{-}$of the vector fields of the form $\bar{v}_{\mu}=\epsilon_{\mu \mu^{\prime}} L_{\mu^{\prime}}^{\dagger}$. All epsilon tensors cancel after taking the trace in (9.17) and we arrive at the same formula as for $D_{+}$but with the replacement $\Phi \rightarrow-\Phi$. The spaces $V_{+}$and $V_{-}$are orthogonal, and $V_{+}+V_{-}=V$ is the space of all vector fields on $M$ (we neglect possible zero modes). Therefore,

$\delta\left[\zeta\left(s, D_{+}\right)-\zeta\left(s, D_{-}\right)\right]=2 s\left[\zeta(s, \delta \Phi, H+\bar{H})-\zeta\left(s, \delta \Phi, D_{+}\right)-\zeta\left(s, \delta \Phi, D_{-}\right)\right]$.

The operator

$$
(H+\bar{H})_{\mu \nu}=\delta_{\mu \nu} D_{+}+\Phi_{, \mu \nu}
$$


is of Laplace type. We can now act in the standard manner to obtain

$$
\delta\left[\zeta^{\prime}\left(0, D_{+}\right)-\zeta^{\prime}\left(s, D_{-}\right)\right]=2\left[a_{2}(\delta \Phi, H+\bar{H})-a_{2}\left(\delta \Phi, D_{+}\right)-a_{2}\left(\delta \Phi, D_{-}\right)\right] .
$$

The right hand side can be evaluated by using (4.27). We leave it is an exercise to show that the variation (9.21) is zero (alternatively, one may look up in [409]). This leads us to the conclusion that

$$
\zeta^{\prime}\left(0, D_{+}\right)-\zeta^{\prime}\left(0, D_{-}\right)=-\ln \operatorname{det} D_{+}+\ln \operatorname{det} D_{-}=0
$$

In the one-dimensional case a similar relation may be obtained by methods of supersymmetric quantum mechanics. In this simplest case, and in higher dimensions if $\Phi$ depends on one of the coordinates only, $D_{+}$and $D_{-}$are isospectral up to zero modes and (9.22) follows immediately. In two dimensions, $D_{+}$and $D_{-}$are not isospectral in general. Although $D_{+}$and $D_{-}$have coinciding determinants, other spectral functions can be different.

Extensions of (9.22) to the case of matrix-valued $\Phi$, manifolds with boundaries, and the dilaton-Maxwell theory in four dimensions can be found in [409]. A generalisation to arbitrary dimension has been achieved recently [235]. Let $A_{p}$ be a $p$-form field with the field strength $F=d A_{p}$. Consider the classical action

$$
\mathcal{L}=\int_{M} e^{-2 \Phi} F \wedge \star F
$$

where $\star$ is the Hodge duality operator. Such actions appear, for example, in extended supergravities [133] and bosonic M-theory [277]. Instead of the dilaton, also a tachyon coupling may appear [300]. We are interested in the symmetry properties of the effective action under the transformation $p \rightarrow$ $n-p-2, \Phi \rightarrow-\Phi$. In higher dimensional supergravity theories this is a part of the $S$-duality transformation.

It is convenient to define the twisted exterior derivative

$$
d_{\Phi}:=e^{-\Phi} d e^{\Phi}
$$

and the associated twisted coderivative and twisted Laplacian

$$
\delta_{\Phi}:=e^{\Phi} \delta e^{-\Phi}, \quad \Delta_{\Phi}:=\left(d_{\Phi}+\delta_{\Phi}\right)^{2} .
$$

Since $d_{\Phi}^{2}=0$ we have an elliptic complex. The restriction of $\Delta_{\Phi}$ on the space of $p$ forms will be denoted by $\Delta_{\Phi}^{p}$. This twisted de Rham complex was introduced by Witten in the context of Morse theory and supersymmetric quantum mechanics [423].

Any $p$-form can be decomposed as the sum of a twisted exact, twisted co-exact, and twisted harmonic form:

$$
A_{p}=d_{\Phi} A_{p-1}+\delta_{\Phi} A_{p+1}+\gamma_{p}, \quad \Delta_{\Phi} \gamma_{p}=0 .
$$

The projections on the spaces of twisted exact and twisted co-exact forms will be denoted by the subscripts $\|$ and $\perp$ respectively. 
We assume that the fields $\tilde{A}_{p}=e^{-\Phi} A_{p}$ have a standard Gaussian measure and are to be considered as fundamental fields in the path integral. The action given in eq. (9.23) is invariant under the gauge transformation which sends $\tilde{A}_{p}$ to $\tilde{A}_{p}+d_{\phi} \tilde{A}_{p-1}$. This means that the $p$-forms which are $d_{\phi}$ exact have to be excluded from the path integral, but that a Jacobian factor corresponding to the ghost fields $\tilde{A}_{p-1}$ has to be included in the path integral measure. Next we note that $d_{\phi}$-exact $(p-1)$-forms do not generate a non-trivial transformation of $\tilde{A}_{p}$. Hence, such fields must be excluded from the ghost sector. Then we have to include "ghosts for ghosts". This goes on until the zero forms have been reached. By giving these arguments an exact meaning, one arrives at the Faddeev-Popov approach to quantisation of the $p$-form actions $[175,386$, $349,44]$. We note that the procedure of $[349,44]$ is valid also in the presence of a dilaton interaction if one simply replaces the ordinary derivatives by the twisted ones. As a result, we have the following expression for the effective action:

$$
W_{p}(\Phi)=\frac{1}{2} \sum_{k=0}^{p}(-1)^{p+k} \ln \operatorname{det}\left(\left.\Delta_{\Phi}^{k}\right|_{\perp}\right)+W_{p}^{\text {top }} .
$$

$W_{p}^{\text {top }}$ depends on certain topological characteristics of the manifold (the Betti numbers). We shall neglect $W_{p}^{\text {top }}$ and some other topological contributions in what follows.

By combinatorial arguments, similar to the presented above, one can show that [235]:

$$
\delta\left(W_{p}(\Phi)-W_{n-p-2}(-\Phi)\right)=\sum_{k=0}^{m}(-1)^{p+k} a_{n}\left(\delta \Phi, \Delta_{\Phi}^{k}\right) .
$$

One should distinguish between $\delta \Phi$ (which is a variation of the dilaton) and $\delta_{\Phi}$ (which is a twisted co-derivative). Therefore, we have related the variation of the effective actions with respect to $\Phi$ to a combination of the heat kernel coefficients which is called the supertrace of the twisted de Rham complex. A somewhat surprising feature of the supertrace is that it can be calculated for any $n$, with or without boundaries [235]. For example, the volume term in (9.28) does not depend on $\Phi$ and, therefore, is the standard Euler density which is given by

$$
\mathbf{E}_{n}:=(4 \pi)^{-\bar{n}} \frac{1}{2^{\bar{n}} \bar{n} !} \epsilon^{i_{1} \ldots i_{m}} \epsilon^{j_{1} \ldots j_{n}} R_{i_{1} i_{2} j_{2} j_{1}} \ldots R_{i_{n-1} i_{n} j_{n} j_{n-1}}
$$

(with $\bar{n}=n / 2$ ) for $n$ even. $\mathbf{E}_{n}=0$ for $n$ odd.

It is known $[213,44]$ that for $\Phi=0$ the dual theories are quantum equivalent:

$$
W_{p}(0)-W_{n-p-2}(0)=0 .
$$

By using this equation as an "initial condition" we can integrate the variation (9.28) to obtain:

$$
W_{p}(\Phi)-W_{n-p-2}(-\Phi)=(-1)^{p} \int_{M} d^{n} x \sqrt{g} \Phi \mathbf{E}_{n}
$$


Further terms $\sum_{p}(-1)^{p} a_{k}\left(x, \Delta_{\Phi}^{p}\right)$ are of some interest in mathematics. They can be calculated $[246,234]$ for $k \leq n+2$ and any $n$. 


\section{Conclusions}

Here we present a short guide in this report. In other words, we are going to answer the following question. What should one do if one likes to calculate one-loop counterterms, anomalies, an expansion term in the effective action, or something else which is defined by the heat kernel expansion? The first step is to find the bulk part of the variation of the classical action (1.2) and corresponding operator $D$. Next one has to bring this operator to the canonical form (2.2) with the help of (2.1), (2.3) and (2.4). However, one can first consult sec. 3. Probably, relevant expressions can be found there. If the problem in question contains neither boundaries nor singularities, one can look in sec. 4 for an expression for the heat kernel coefficient, or for a reference, or for a method. In the case of the boundaries one has to proceed with sec. 5 , in the case of singularities - with sec. 6 . Relations between the heat kernel coefficients and quantum anomalies can be found in sec. 7. The results going beyond the standard heat kernel expansion are collected in sec. 8. In particular, in this section we explain how one can extract leading non-localities from the effective action, and what the heat kernel looks like if the background is approximately covariantly constant (in this context we also consider invariant operators on homogeneous spaces). Exact results for the effective action which can be obtained with the help of the heat kernel expansion are reviewed in sec. 9 .

The Casimir energy is one of the most "classical" applications of the heat kernel and zeta function technique (cf. [70]). It follows from the locality of the heat kernel expansion that the divergences in the Casimir energy are given by volume and surface integrals of some local invariants. Therefore, if the boundaries are being moved in such a way that the boundary values of the background fields remain unchanged, the boundary contributions to the divergences also remain constant. This leads to the well known conclusion [79] that there are no boundary divergences in the Casimir force (which is defined, roughly speaking as a variation of the Casimir energy under infinitesimal translations of the boundary). Consequently, one can assign a unique value to the Casimir force (see, e.g., [324]). This observation, however does not mean that the quantum field theory on a manifold with boundary is finite. In general, some surface counterterms are required (at one-loop order they may be read off from sec. 5). Moreover, if the background field are non-trivial, the boundary divergences will not be constant. Similar arguments created certain scepticism towards reliability of the Casimir energy calculation [252]. This point has not been settled so far for a "generic" theory. We may add that in supersymmetric theories cancellations between divergences in the bosonic and fermionic sectors appear if the boundary terms are considered together with the volume terms [84], therefore, separation of boundary and volume contributions is not always natural for that theories. Renormalization of self-interacting theories on manifolds with boundaries was considered in [394,351,317, 420,54] where

one can find further references. Some aspects of the relationship between the 
Casimir energy calculations and the heat kernel coefficients have been clarified recently by Fulling [225].

Of course, not everything can be found in this report. Several topics are very close to the subject of this review, but are not included.

(1) The heat kernel expansion can be successfully applied to quantum field theory at finite temperature [166]. A new interesting development in this field is related to the so called non-linear spectral problem [228] (see [229] for an overview).

(2) The heat kernel expansion has interesting applications to integrable models and, in particular, to the KdV hierarchies (see [135] for an elementary introduction).

(3) Recently some attention has been attracted $[162,171,384,33]$ to the so called N/D or Zaremba problem which appears when one defines Neumann and Dirichlet boundary conditions on two (intersecting) components of the boundary. It is unclear whether this problem may have applications to quantum theory.

(4) Instead of considering asymptotics of the heat trace $\operatorname{Tr}\left(f e^{-t D}\right)$ one can also consider an asymptotic expansion for individual matrix elements of the heat kernel $\left\langle f_{1}, e^{-t D} f_{2}\right\rangle$ (which are called the heat content asymptotics since they remind short time asymptotics of the total heat content in a manifold with the specific heat $f_{1}$ and the initial temperature distribution $f_{2}$ ). Such asymptotics do not contain negative powers of $t$. More details can be found in [297].

(5) Many results on the heat kernel asymptotics can be extended to higherorder differential operators (see, e.g., [238, 268]) and to differential operators in superspace [101].

Although quite a lot is already known about the heat kernel expansion, many interesting problems still remain open. There are many opportunities to extend and generalise the results presented in this report. This refers especially to the material of sec. 5-9 where one could add new types of the operators, boundary conditions, geometries, and singularities. There is a completely new field of research related to the heat kernel expansion where very little has been done so far. This is an extension to non-commutative geometry. This problem is an especially intriguing one since one can expect very unusual properties of the spectral functions because of very unusual properties of corresponding field theories in the ultra violet asymptotics.

\section{Acknowledgements}

I am grateful to my collaborators S. Alexandrov, M. Bordag, T. Branson, E. Elizalde, H. Falomir, P. Gilkey, D. Grumiller, K. Kirsten, W. Kummer, H. Liebl, V. Lyakhovsky, V. Marachevsky, Yu. Novozhilov, M. Santangelo, N. Shtykov, P. van Nieuwenhuizen and A. Zelnikov who contributed in many ways to the material presented in this report. I have benefited from enlight- 
ening discussions with I. Avramidi, A. Barvinsky, G. Esposito, D. Fursaev, G. Grubb, A. Kamenshchik, V. Nesterenko and with my friends and colleagues at St. Petersburg, Leipzig and Vienna.

I am grateful to all readers who suggested their comments on the previous version of the manuscript. I also thank the referee for useful critical remarks.

This work has been supported by Project BO 1112/12-1 of the Deutsche Forschungsgemeinschaft. I am grateful to E. Zeidler for his kind hospitality at the Max Planck Institute for Mathematics in the Sciences where a part of this work has been done. 


\section{References}

[1] A. Abouelsaood, C. G. Callan, C. R. Nappi, and S. A. Yost, "Open strings in background gauge fields," Nucl. Phys. B280 (1987) 599.

[2] A. A. Actor and I. Bender, "Casimir effect for soft boundaries," Phys. Rev. D52 (1995) 3581-3590.

[3] S. L. Adler, "Axial vector vertex in spinor electrodynamics," Phys. Rev. 177 (1969) 2426-2438.

[4] S. L. Adler and T. Piran, "Flux confinement in the leading logarithm model," Phys. Lett. B113 (1982) 405.

[5] S. Albeverio, Z. Brzezniak, and L. Dabrowski, "Time-dependent propagator with point interaction," J. Phys. A 19 (1994) 4933-4943.

[6] S. Albeverio, F. Gesztesy, R. Hoegh-Krohn, and H. Holden, Solvable models in quantum mechanics. Springer, New York, 1988.

[7] S. Alexandrov and D. Vassilevich, "Heat kernel for non-minimal operators on a Kaehler manifold," J. Math. Phys. 37 (1996) 5715-5718, hep-th/9601090.

[8] B. Allen, "The graviton propagator in de Sitter space," Phys. Rev. D34 (1986) 3670.

[9] A. Alonso Izquierdo, W. Garcia Fuertes, M. A. Gonzalez Leon, and J. Mateos Guilarte, "Generalized zeta functions and one-loop corrections to quantum kink masses," Nucl. Phys. B635 (2002) 525-557, hep-th/0201084.

[10] O. Alvarez, "Theory of strings with boundaries: Fluctuations, topology, and quantum geometry," Nucl. Phys. B216 (1983) 125.

[11] O. Alvarez, "Conformal anomalies and the index theorem," Nucl. Phys. B286 (1987) 175.

[12] P. Amsterdamski, A. L. Berkin, and D. J. O'Connor, "B(8) 'hamidew' coefficient for a scalar field," Class. Quant. Grav. 6 (1989) 1981.

[13] A. Andrianov and L. Bonora, "Finite - mode regularization of the fermion functional integral," Nucl. Phys. B233 (1984) 232.

[14] A. Andrianov and L. Bonora, "Finite mode regularization of the fermion functional integral. 2," Nucl. Phys. B233 (1984) 247.

[15] T. Appelquist and A. Chodos, "Quantum effects in Kaluza-Klein theories," Phys. Rev. Lett. 50 (1983) 141.

[16] T. Appelquist and A. Chodos, "The quantum dynamics of Kaluza-Klein theories," Phys. Rev. D28 (1983) 772.

[17] J. S. Apps and J. S. Dowker, "The c(2) heat-kernel coefficient in the presence of boundary discontinuities," Class. Quant. Grav. 15 (1998) 1121-1139, hep-th/9712019. 
[18] M. Atiyah, R. Bott, and V. K. Patodi, "On the heat equation and the index theorem," Invent. Math. 19 (1973) 279-330. Errata: ibid 28 (175) 277-280.

[19] M. F. Atiyah, V. K. Patodi, and I. M. Singer, "Spectral asymmetry and Riemannian Geometry 1," Math. Proc. Cambridge Phil. Soc. 77 (1975) 43.

[20] M. F. Atiyah, V. K. Patodi, and I. M. Singer, "Spectral asymmetry and Riemannian geometry 2," Math. Proc. Cambridge Phil. Soc. 78 (1976) 405.

[21] M. F. Atiyah, V. K. Patodi, and I. M. Singer, "Spectral asymmetry and Riemannian Geometry 3," Math. Proc. Cambridge Phil. Soc. 79 (1980) 71.

[22] M. F. Atiyah and I. M. Singer, "The index of elliptic operators on compact manifolds," Bull. Amer. Math. Soc. 69 (1963) 422-433.

[23] E. Aurell and P. Salomonsen, "On functional determinants of Laplacians in polygons and simplicial complexes," Commun. Math. Phys. 165 (1994) 233-259.

[24] I. G. Avramidi, "Covariant methods of studying the nonlocal structure of an effective action," Sov. J. Nucl. Phys. 49 (1989) 735-739.

[25] I. G. Avramidi, "The covariant technique for the calculation of the heat kernel asymptotic expansion," Phys. Lett. B238 (1990) 92.

[26] I. G. Avramidi, "The covariant technique for calculation of one loop effective action," Nucl. Phys. B355 (1991) 712-754. Erratum:ibid. B509:577-558, 1998.

[27] I. G. Avramidi, "A new algebraic approach for calculating the heat kernel in gauge theories," Phys. Lett. B305 (1993) 27-34.

[28] I. G. Avramidi, "The heat kernel on symmetric spaces via integrating over the group of isometries," Phys. Lett. B336 (1994) 171-177, hep-th/9509079.

[29] I. G. Avramidi, "Covariant algebraic method for calculation of the lowenergy heat kernel," J. Math. Phys. 36 (1995) 5055-5070, hep-th/9503132.

[30] I. G. Avramidi, "A new algebraic approach for calculating the heat kernel in quantum gravity," J. Math. Phys. 37 (1996) 374-394, hep-th/9406047.

[31] I. G. Avramidi, Heat kernel and quantum gravity. Springer, Berlin, 2000.

[32] I. Avramidi and T. Branson, "Heat kernel asymptotics of operators with non-Laplace principal part," Rev. Math. Phys. 13 (2001) 847-890.

[33] I. G. Avramidi, "Heat kernel asymptotics of Zaremba boundary value problem," math-ph/0110020.

[34] I. G. Avramidi, "Heat kernel approach in quantum field theory," Nucl. Phys. Proc. Suppl. 104 (2002) 3-32, math-ph/0107018. 
[35] I. G. Avramidi and G. Esposito, "Heat kernel asymptotics of Gilkey-Smith boundary value problem," in Trends in mathematical physics, V. Alexiades and G. Siopsis, eds., AMS/IP Studies in Advanced Mathematics, pp. 15-34. International Press, Cambridge, Mass., 1998. math-ph/9812010.

[36] I. G. Avramidi and G. Esposito, "Lack of strong ellipticity in euclidean quantum gravity," Class. Quant. Grav. 15 (1998) 1141-1152, hep-th/9708163

[37] I. G. Avramidi and G. Esposito, "New invariants in the one-loop divergences on manifolds with boundary," Class. Quant. Grav. 15 (1998) 281-297, hep-th/9701018.

[38] I. G. Avramidi and G. Esposito, "Gauge theories on manifolds with boundary," Commun. Math. Phys. 200 (1999) 495-543, hep-th/9710048.

[39] I. G. Avramidi, G. Esposito, and A. Y. Kamenshchik, "Boundary Operators in Euclidean Quantum Gravity," Class. Quant. Grav. 13 (1996) 2361-2374, hep-th/9603021

[40] C. Bachas, J. de Boer, R. Dijkgraaf, and H. Ooguri, "Permeable conformal walls and holography," J. High Energy Phys. 06 (2002) 027, hep-th/0111210

[41] R. Balbinot, A. Fabbri, and I. Shapiro, "Vacuum polarization in Schwarzschild space-time by anomaly induced effective actions," Nucl. Phys. B559 (1999) 301-319, hep-th/9904162.

[42] R. Balian and C. Bloch, "Distribution of eigenfrequencies of the wave equation in a finite domain: 1 . Three-dimensional problem with smooth boundary sirface," Ann. Phys. 60 (1979) 401.

[43] R. Balian and B. Duplantier, "Electromagnetic waves near perfect conductors. 1. multiple scattering expansions. distribution of modes," Ann. Phys. 104 (1977) 300.

[44] J. L. F. Barbon, "Generalized Abelian S duality and coset constructions," Nucl. Phys. B452 (1995) 313-330, hep-th/9506137.

[45] M. Barriola and A. Vilenkin, "Gravitational field of a global monopole," Phys. Rev. Lett. 63 (1989) 341.

[46] A. Barut and R. Raczka, Theory of group represenations and applications. PWN, Warszawa, 1977.

[47] A. O. Barvinsky, "The wave function and the effective action in quantum cosmology: Covariant loop expansion," Phys. Lett. B195 (1987) 344-348.

[48] A. O. Barvinsky, Y. V. Gusev, V. F. Mukhanov, and D. V. Nesterov, "Nonperturbative late time asymptotics for heat kernel in gravity theory," hep-th/0306052. 
[49] A. O. Barvinsky, Y. V. Gusev, G. A. Vilkovisky, and V. V. Zhytnikov, "Asymptotic behaviors of the heat kernel in covariant perturbation theory," J. Math. Phys. 35 (1994) 3543-3559, gr-qc/9404063

[50] A. O. Barvinsky, Y. V. Gusev, G. A. Vilkovisky, and V. V. Zhytnikov, "The basis of nonlocal curvature invariants in quantum gravity theory. (third order.)," J. Math. Phys. 35 (1994) 3525-3542, gr-qc/9404061.

[51] A. O. Barvinsky, A. Y. Kamenshchik, and I. P. Karmazin, "One loop quantum cosmology: Zeta function technique for the Hartle-Hawking wave function of the universe," Annals Phys. 219 (1992) 201-242.

[52] A. O. Barvinsky, A. Y. Kamenshchik, I. P. Karmazin, and I. V. Mishakov, "One loop quantum cosmology: The contributions of matter fields to the wave function of the universe," Class. Quant. Grav. 9 (1992) L27-L32.

[53] A. O. Barvinsky and V. F. Mukhanov, "New nonlocal effective action," Phys. Rev. D66 (2002) 065007, hep-th/0203132.

[54] A. O. Barvinsky and S. N. Solodukhin, "Non-minimal coupling, boundary terms and renormalization of the Einstein-Hilbert action and black hole entropy," Nucl. Phys. B479 (1996) 305-318, gr-qc/9512047.

[55] A. O. Barvinsky and G. A. Vilkovisky, "The generalized Schwinger-DeWitt technique in gauge theories and quantum gravity," Phys. Rept. 119 (1985) $1-74$.

[56] A. O. Barvinsky and G. A. Vilkovisky, "Beyond the Schwinger-DeWitt technique: Converting loops into trees and in-in currents," Nucl. Phys. B282 (1987) 163-188.

[57] A. O. Barvinsky and G. A. Vilkovisky, "Covariant perturbation theory. 2: Second order in the curvature. General algorithms," Nucl. Phys. B333 (1990) 471-511.

[58] A. O. Barvinsky and G. A. Vilkovisky, "Covariant perturbation theory. 3: Spectral representations of the third order form-factors," Nucl. Phys. B333 (1990) 512-524.

[59] I. A. Batalin, S. G. Matinian, and G. K. Savvidy, "Vacuum polarization by a source - free gauge field," Sov. J. Nucl. Phys. 26 (1977) 214.

[60] J. S. Bell and R. Jackiw, "A PCAC puzzle: $\pi^{0} \rightarrow \gamma \gamma$ in the sigma model," Nuovo Cim. A60 (1969) 47-61.

[61] C. G. Beneventano, M. De Francia, and E. M. Santangelo, "Dirac fields in the background of a magnetic flux string and spectral boundary conditions," Int. J. Mod. Phys. A14 (1999) 4749-4762, hep-th/9809081.

[62] C. G. Beneventano, E. M. Santangelo, and A. Wipf, "Spectral asymmetry for bag boundary conditions," hep-th/0205199. 
[63] F. A. Berezin and L. D. Faddeev, "A remark on Schrödinger's equation with a singular potential," Soviet Math. Dokl. 2 (1961) 372-375.

[64] F. Bernasconi, G. M. Graf, and D. Hasler, "The heat kernel expansion for the electromagnetic field in a cavity," math-ph/0302035

[65] R. A. Bertelmann, Anomalies in Quantum Field Theory. Clarendon Press, Oxford, 1996.

[66] E. R. Bezerra de Mello, V. B. Bezerra, and N. R. Khusnutdinov, "Ground state energy of massive scalar field inside a spherical region in the global monopole background," J. Math. Phys. 42 (2001) 562-581, hep-th/0012264.

[67] D. Birmingham, "Conformal anomaly in spherical space-times," Phys. Rev. D36 (1987) 3037-3047.

[68] D. Birmingham, R. Kantowski, and K. A. Milton, "Scalar and spinor casimir energies in even dimensional Kaluza-Klein spaces of the form $M^{4} \times S^{N_{1}} \times S^{N_{2}} \times \ldots, "$ Phys. Rev. D38 (1988) 1809.

[69] N. D. Birrell and P. C. W. Davies, Quantum Fields in Curved Space. Cambridge Univ. Press, Cambridge, 1982.

[70] S. Blau, M. Visser, and A. Wipf, "Zeta functions and the Casimir energy," Nucl. Phys. B310 (1988) 163.

[71] S. K. Blau, M. Visser, and A. Wipf, "Analytical results for the effective action," Int. J. Mod. Phys. A6 (1991) 5409-5433.

[72] M. Bordag, E. Elizalde, and K. Kirsten, "Heat kernel coefficients of the Laplace operator on the D- dimensional ball," J. Math. Phys. 37 (1996) 895-916, hep-th/9503023

[73] M. Bordag, E. Elizalde, K. Kirsten, and S. Leseduarte, "Casimir energies for massive fields in the bag," Phys. Rev. D56 (1997) 4896-4904, hep-th/9608071.

[74] M. Bordag, H. Falomir, E. M. Santangelo, and D. V. Vassilevich, "Boundary dynamics and multiple reflection expansion for Robin boundary conditions," Phys. Rev. D65 (2002) 064032, hep-th/0111073.

[75] M. Bordag, B. Geyer, K. Kirsten, and E. Elizalde, "Zeta function determinant of the Laplace operator on the D- dimensional ball," Commun. Math. Phys. 179 (1996) 215-234, hep-th/9505157.

[76] M. Bordag and K. Kirsten, "Heat kernel coefficients and divergencies of the Casimir energy for the dispersive sphere," Int. J. Mod. Phys. A17 (2002) 813-819, hep-th/0110217

[77] M. Bordag, K. Kirsten, and D. Vassilevich, "On the ground state energy for a penetrable sphere and for a dielectric ball," Phys. Rev. D59 (1999) 085011, hep-th/9811015 
[78] M. Bordag, K. Kirsten, and D. V. Vassilevich, "Path integral quantization of electrodynamics in dielectric media," J. Phys. A31 (1998) 2381-2389, hep-th/9709084

[79] M. Bordag, U. Mohideen, and V. M. Mostepanenko, "New developments in the Casimir effect," Phys. Rept. 353 (2001) 1-205, quant-ph/0106045

[80] M. Bordag and I. G. Pirozhenko, "The heat kernel coefficients for the dielectric cylinder," Phys. Rev. D64 (2001) 025019, hep-th/0102193

[81] M. Bordag, D. Vassilevich, H. Falomir, and E. M. Santangelo, "Multiple reflection expansion and heat kernel coefficients," Phys. Rev. D64 (2001) 045017, hep-th/0103037.

[82] M. Bordag and D. V. Vassilevich, "Heat kernel expansion for semitransparent boundaries," J. Phys. A32 (1999) 8247-8259, hep-th/9907076.

[83] M. Bordag and D. V. Vassilevich, "Casimir force between Chern-Simons surfaces," Phys. Lett. A268 (2000) 75-80, hep-th/9911179.

[84] M. Bordag, A. S. Goldhaber, P. van Nieuwenhuizen, and D. Vassilevich, "Heat kernels and zeta-function regularization for the mass of the susy kink," Phys. Rev. D66 (2002) 125014, hep-th/0203066.

[85] M. Bordag, K. Kirsten, and S. Dowker, "Heat kernels and functional determinants on the generalized cone," Commun. Math. Phys. 182 (1996) 371-394, hep-th/9602089

[86] R. Bousso and S. W. Hawking, "Trace anomaly of dilaton coupled scalars in two dimensions," Phys. Rev. D56 (1997) 7788-7791, hep-th/9705236.

[87] E. Braaten, T. L. Curtright, and C. K. Zachos, "Torsion and geometrostasis in nonlinear sigma models," Nucl. Phys. B260 (1985) 630.

[88] T. Branson and P. Gilkey, "Residues of the eta function for an operator of Dirac type," J. Funct. Anal. 108 (1992) 47.

[89] T. Branson and P. Gilkey, "Residues of the eta function for an operator of Dirac type with local boundary condtitons," Differential Geom. Appl. 2 (1992) 249.

[90] T. P. Branson and P. B. Gilkey, "The asymptotics of the Laplacian on a manifold with boundary," Commun. Part. Diff. Equat. 15 (1990) 245-272.

[91] T. P. Branson, P. B. Gilkey, K. Kirsten, and D. V. Vassilevich, "Heat kernel asymptotics with mixed boundary conditions," Nucl. Phys. B563 (1999) 603, hep-th/9906144

[92] T. P. Branson, P. B. Gilkey, and B. Ørsted, "Leading terms in the heat invariants," Proc. Amer. Math. Soc. 109 (1990) 437-450.

[93] T. P. Branson, P. B. Gilkey, and D. V. Vassilevich, "The asymptotics of the laplacian on a manifold with boundary. 2," Boll. Union. Mat. Ital. 11B (1997) 39-67, hep-th/9504029 
[94] T. P. Branson, P. B. Gilkey, and D. V. Vassilevich, "Vacuum expectation value asymptotics for second order differential operators on manifolds with boundary," J. Math. Phys. 39 (1998) 1040, hep-th/9702178.

[95] G. E. Brown and M. Rho, "The little bag," Phys. Lett. B82 (1979) 177-180.

[96] L. S. Brown and J. P. Cassidy, "Stress tensor trace anomaly in a gravitational metric: General theory, Maxwell field," Phys. Rev. D15 (1977) 2810.

[97] J. Brüning, "Heat equation asymptotics for singular sturm-liouville operators," Math. Ann. 268 (1984) 173.

[98] J. Brüning and R. Seeley, "The resolvent expansion for second order regular singular operators," J. Func. Anal. 73 (1987) 369-429.

[99] I. L. Buchbinder, "Renormalization group equations in curved space-time," Theor. Math. Phys. 61 (1984) 1215-1219.

[100] I. L. Buchbinder, V. P. Gusynin, and P. I. Fomin, "Functional determinants and effective action for conformal scalar and spinor fields in external gravitational field," Sov. J. Nucl. Phys. 44 (1986) 534-539.

[101] I. L. Buchbinder and S. M. Kuzenko, "Matter superfields in external supergravity: Green functions, effective action and superconformal anomalies," Nucl. Phys. B274 (1986) 653-684.

[102] I. L. Buchbinder, P. M. Lavrov, and S. D. Odintsov, "Unique effective action in Kaluza-Klein quantum theories and spontaneous compactification," Nucl. Phys. B308 (1988) 191-202.

[103] I. L. Buchbinder and S. D. Odintsov, "Asymptotical properties of nonabelian gauge theories in external gravitational fields," Yad. Fiz. 40 (1984) $1338-1343$.

[104] I. L. Buchbinder and S. D. Odintsov, "Asymptotical conformal invariance in curved space-time," Lett. Nuovo Cim. 42 (1985) 379-381.

[105] I. L. Buchbinder and S. D. Odintsov, "Effective action in multidimensional supergravities and spontaneous compactification," Int. J. Mod. Phys. A4 (1988) 1859-1870.

[106] I. L. Buchbinder and S. D. Odintsov, "Effective action in multidimensional (super)gravities and spontaneous compactification. (quantum aspects of kaluza- klein theories)," Fortsch. Phys. 37 (1989) 225-259.

[107] I. L. Buchbinder, S. D. Odintsov, and I. L. Shapiro, Effective action in quantum gravity. IOP,Bristol, UK, 1992.

[108] I. L. Buchbinder and J. J. Wolfengaut, "Renormalization group equations and effective action in curved space-time," Class. Quant. Grav. 5 (1988) $1127-1136$. 
[109] A. A. Bytsenko, "Heat-kernel asymptotics of locally symmetric spaces of rank one and Chern-Simons invariants," Nucl. Phys. Proc. Suppl. 104 (2002) 127-134, hep-th/0108032

[110] A. A. Bytsenko, G. Cognola, L. Vanzo, and S. Zerbini, "Quantum fields and extended objects in space-times with constant curvature spatial section," Phys. Rept. 266 (1996) 1-126, hep-th/9505061.

[111] C. G. Callan, R. F. Dashen, and D. J. Gross, "A theory of hadronic structure," Phys. Rev. D19 (1979) 1826.

[112] C. G. Callan, C. Lovelace, C. R. Nappi, and S. A. Yost, "String loop corrections to beta functions," Nucl. Phys. B288 (1987) 525.

[113] C. G. Callan, E. J. Martinec, M. J. Perry, and D. Friedan, "Strings in background fields," Nucl. Phys. B262 (1985) 593.

[114] H. E. Camblong and C. R. Ordonez, "Renormalized path integral for the two-dimensional delta- function interaction," hep-th/0110176

[115] R. Camporesi, "Harmonic analysis and propagators on homogeneous spaces," Phys. Rept. 196 (1990) 1-134.

[116] P. Candelas, "Vacuum polarization in the presence of dielectric and conducting surfaces," Ann. Phys. 143 (1982) 241.

[117] P. Candelas and S. Weinberg, "Calculation of gauge couplings and compact circumferences from selfconsistent dimensional reduction," Nucl. Phys. B237 (1984) 397.

[118] D. M. Capper and M. J. Duff, "Trace anomalies in dimensional regularization," Nuovo Cim. A23 (1974) 173-183.

[119] H. S. Carslaw and J. C. Jaeger, Conduction of heat in solids. Clarendon Press, Oxford, 1986.

[120] A. Ceresole, P. Pizzochero, and P. van Nieuwenhuizen, "The curved space trace, chiral and einstein anomalies from path integrals, using flat space plane waves," Phys. Rev. D39 (1989) 1567.

[121] P. Chang and J. S. Dowker, "Vacuum energy on orbifold factors of spheres," Nucl. Phys. B395 (1993) 407-432, hep-th/9210013

[122] J. Cheeger, "Spectral geometry of singular Riemannian spaces," J. Diff. Geom. 18 (1983) 575-657.

[123] H. T. Cho and R. Kantowski, "Zeta functions for nonminimal operators," Phys. Rev. D52 (1995) 4588-4599, hep-th/9503188

[124] A. Chodos, R. L. Jaffe, K. Johnson, and C. B. Thorn, "Baryon structure in the bag theory," Phys. Rev. D10 (1974) 2599.

[125] A. Chodos, R. L. Jaffe, K. Johnson, C. B. Thorn, and V. F. Weisskopf, "A new extended model of hadrons," Phys. Rev. D9 (1974) 3471-3495. 
[126] A. Chodos and C. B. Thorn, "Chiral invariance in the bag theory," Phys. Rev. D12 (1975) 2733.

[127] S. M. Christensen and M. J. Duff, "New gravitational index theorems and supertheorems," Nucl. Phys. B154 (1979) 301.

[128] S. M. Christensen and M. J. Duff, "Quantizing gravity with a cosmological constant," Nucl. Phys. B170 (1980) 480.

[129] G. Cognola, K. Kirsten, and L. Vanzo, "Free and selfinteracting scalar fields in the presence of conical singularities," Phys. Rev. D49 (1994) 1029-1038, hep-th/9308106

[130] G. Cognola and L. Vanzo, "The trace of the heat kernel on a compact hyperbolic 3 orbifold," J. Math. Phys. 35 (1994) 3109-3116, hep-th/9303028

[131] G. Cognola, L. Vanzo, and S. Zerbini, "A new algorithm for asymptotic heat kernel expansion for manifolds with boundary," Phys. Lett. B241 (1990) $381-386$.

[132] G. Cognola and S. Zerbini, "Seeley-De Witt coefficients in a Riemann-Cartan manifold," Phys. Lett. B214 (1988) 70.

[133] E. Cremmer, J. Scherk, and S. Ferrara, "SU(4) invariant supergravity theory," Phys. Lett. B74 (1978) 61.

[134] J. Dai, R. G. Leigh, and J. Polchinski, "New connections between string theories," Mod. Phys. Lett. A4 (1989) 2073-2083.

[135] A. K. Das, Integrable models. World Scientific, Singapore, 1989.

[136] G. De Berredo-Peixoto, "A note on the heat kernel method applied to fermions," Mod. Phys. Lett. A16 (2001) 2463-2468, hep-th/0108223.

[137] M. De Francia, H. Falomir, and M. Loewe, "Finite density and temperature in hybrid bag models," Phys. Rev. C60 (1999) 055203, hep-th/9811046.

[138] M. De Francia, H. Falomir, and E. M. Santangelo, "Free energy of a four-dimensional chiral bag," Phys. Rev. D45 (1992) 2129-2139.

[139] M. De Francia, H. Falomir, and E. M. Santangelo, "Cheshire cat scenario in a (3+1)-dimensional hybrid chiral bag," Phys. Lett. B371 (1996) 285-292, hep-ph/9507347.

[140] L. De Nardo, D. V. Fursaev, and G. Miele, "Heat-kernel coefficients and spectra of the vector Laplacians on spherical domains with conical singularities," Class. Quant. Grav. 14 (1997) 1059-1078, hep-th/9610011.

[141] P. D. D'Eath and G. Esposito, "Local boundary conditions for the Dirac operator and one loop quantum cosmology," Phys. Rev. D43 (1991) $3234-3248$, gr-qc/9507004 
[142] P. D. D'Eath and G. Esposito, "Spectral boundary conditions in one loop quantum cosmology," Phys. Rev. D44 (1991) 1713-1721, gr-qc/9507005.

[143] T. DeGrand, R. L. Jaffe, K. Johnson, and J. E. Kiskis, "Masses and other parameters of the light hadrons," Phys. Rev. D12 (1975) 2060.

[144] S. Deser, M. J. Duff, and C. J. Isham, "Nonlocal conformal anomalies," Nucl. Phys. B111 (1976) 45.

[145] S. Deser and R. Jackiw, "Classical and quantum scattering on a cone," Commun. Math. Phys. 118 (1988) 495.

[146] S. Deser, R. Jackiw, and G. 't Hooft, "Three-dimensional Einstein gravity: Dynamics of flat space," Ann. Phys. 152 (1984) 220.

[147] B. S. DeWitt, Dynamical Theory of Groups and Fields. Gordon and Breach, New York, 1965.

[148] B. S. DeWitt, "Quantum theory of gravity. 1. the canonical theory," Phys. Rev. 160 (1967) 1113-1148.

[149] B. S. DeWitt, "Quantum theory of gravity. ii. the manifestly covariant theory," Phys. Rev. 162 (1967) 1195-1239.

[150] B. S. DeWitt, "Quantum theory of gravity. iii. applications of the covariant theory," Phys. Rev. 162 (1967) 1239-1256.

[151] W. Dittrich and M. Reuter, "Effective QCD lagrangian with zeta function regularization," Phys. Lett. B128 (1983) 321.

[152] B. P. Dolan, "The spectrum of the Dirac operator on coset spaces with homogeneous gauge fields," hep-th/0304037.

[153] J. S. Dowker, "Quantum mechanics on group space and Huygens' principle," Ann. Phys. 62 (1971) 361-382.

[154] J. S. Dowker, "Quantum field theory on a cone," J. Phys. A10 (1977) $115-124$.

[155] J. S. Dowker, "Heat kernel expansion on a generalized cone," J. Math. Phys. 30 (1989) 770.

[156] J. S. Dowker, "Functional determinants on spheres and sectors," J. Math. Phys. 35 (1994) 4989-4999, hep-th/9312080.

[157] J. S. Dowker, "A note on Polyakov's nonlocal form of the effective action," Class. Quant. Grav. 11 (1994) L7-L10, hep-th/9309127.

[158] J. S. Dowker, "Robin conditions on the Euclidean ball," Class. Quant. Grav. 13 (1996) 585-610, hep-th/9506042.

[159] J. S. Dowker, "Spin on the 4 ball," Phys. Lett. B366 (1996) 89-94, hep-th/9508082 
[160] J. S. Dowker, "Conformal anomaly in 2d dilaton-scalar theory," Class. Quant. Grav. 15 (1998) 1881-1884, hep-th/9802029.

[161] J. S. Dowker, "Divergences in the casimir energy," hep-th/0006138.

[162] J. S. Dowker, "The N U D problem," hep-th/0007129

[163] J. S. Dowker and J. S. Apps, "Further functional determinants," Class. Quant. Grav. 12 (1995) 1363-1383, hep-th/9502015.

[164] J. S. Dowker, J. S. Apps, K. Kirsten, and M. Bordag, "Spectral invariants for the Dirac equation on the d-Ball with various boundary conditions," Class. Quant. Grav. 13 (1996) 2911-2920, hep-th/9511060.

[165] J. S. Dowker and R. Critchley, "Effective Lagrangian and energy momentum tensor in de Sitter space," Phys. Rev. D13 (1976) 3224.

[166] J. S. Dowker and G. Kennedy, "Finite temperature and boundary effects in static space- times," J. Phys. A11 (1978) 895.

[167] J. S. Dowker and K. Kirsten, "Heat-kernel coefficients for oblique boundary conditions," Class. Quant. Grav. 14 (1997) L169-L175, hep-th/9706129.

[168] J. S. Dowker and K. Kirsten, "The a(3/2) heat kernel coefficient for oblique boundary conditions," Class. Quant. Grav. 16 (1999) 1917-1936, hep-th/9806168

[169] J. S. Dowker and K. Kirsten, "Smeared heat-kernel coefficients on the ball and generalized cone," J. Math. Phys. 42 (2001) 434-452, hep-th/9803094.

[170] J. S. Dowker and J. P. Schofield, "Conformal transformations and the effective action in the presence of boundaries," J. Math. Phys. 31 (1990) 808.

[171] J. S. Dowker, P. Gilkey, and K. Kirsten, "On properties of the asymptotic expansion of the heat trace for the N/D problem," Int. J. Math. 12 (2001) 505-518, hep-th/0010199

[172] S. Dowker, P. Gilkey, and K. Kirsten, "Heat asymptotics with spectral boundary conditions," in Geometric Aspects of Partial Differential Equations, vol. 242 of Contemporary Mathematics, pp. 107-124. AMS, 1999. hep-th/0004020.

[173] I. Drozdov, "Vacuum polarization by a magnetic flux of special rectangular form," hep-th/0210282.

[174] M. J. Duff, "Twenty years of the Weyl anomaly," Class. Quant. Grav. 11 (1994) 1387-1404, hep-th/9308075

[175] M. J. Duff and P. van Nieuwenhuizen, "Quantum inequivalence of different field representations," Phys. Lett. B94 (1980) 179.

[176] G. V. Dunne, "Derivative expansion and soliton masses," Phys. Lett. B467 (1999) 238-246, hep-th/9907208. 
[177] T. Eguchi, P. B. Gilkey, and A. J. Hanson, "Gravitation, gauge theories and differential geometry," Phys. Rept. 66 (1980) 213.

[178] E. Elizalde, Ten physical applications of spectral zeta functions. Springer, Berlin, 1995.

[179] E. Elizalde, M. Bordag, K. Kirsten, and S. Leseduarte, "Casimir energies for massive fields in the bag," J. Phys. A31 (1998) 1743-1759.

[180] E. Elizalde, M. Lygren, and D. V. Vassilevich, "Antisymmetric tensor fields on spheres: functional determinants and non-local counterterms," J. Math. Phys. 37 (1996) 3105-3117, hep-th/9602113

[181] E. Elizalde, M. Lygren, and D. V. Vassilevich, "Zeta function for the Laplace operator acting on forms in a ball with gauge boundary conditions," Commun. Math. Phys. 183 (1997) 645-660, hep-th/9605026.

[182] E. Elizalde, S. Odintsov, A. Romeo, A. A. Bytsenko, and S. Zerbini, Zeta regularization technique with applications. World Scientific, Singapore, 1994.

[183] E. Elizalde and J. Soto, "zeta regularized Lagrangians for massive quarks in constant background mean fields," Ann. Phys. 162 (1985) 192.

[184] E. Elizalde and D. V. Vassilevich, "Heat kernel coefficients for Chern-Simons boundary conditions in QED," Class. Quant. Grav. 16 (1999) 813-823.

[185] E. Elizalde, "Effective Lagrangian for ordinary quarks in a background field," Nucl. Phys. B243 (1984) 398.

[186] E. Elizalde, S. Nojiri, S. D. Odintsov, and S. Ogushi, "Casimir effect in de sitter and anti-de sitter braneworlds," hep-th/0209242.

[187] E. Elizalde, L. Vanzo, and S. Zerbini, "Zeta-function regularization, the multiplicative anomaly and the Wodzicki residue," Commun. Math. Phys. 194 (1998) 613-630, hep-th/9701060

[188] J. Erdmenger and H. Osborn, "Conserved currents and the energy-momentum tensor in conformally invariant theories for general dimensions," Nucl. Phys. B483 (1997) 431-474, hep-th/9605009

[189] G. Esposito, Dirac operators and spectral geometry. Cambridge University Press, Cambridge, 1997.

[190] G. Esposito, "Non-local boundary conditions in Euclidean quantum gravity," Class. Quant. Grav. 16 (1999) 1113-1126, gr-qc/9806057.

[191] G. Esposito, Quantum gravity in four-dimensions. Nova Science, New York, 2001.

[192] G. Esposito and A. Y. Kamenshchik, "Coulomb gauge in one loop quantum cosmology," Phys. Lett. B336 (1994) 324-329, gr-qc/9506062

[193] G. Esposito and A. Y. Kamenshchik, "Mixed boundary conditions in euclidean quantum gravity," Class. Quant. Grav. 12 (1995) 2715-2722, gr-qc/9506092 
[194] G. Esposito, A. Y. Kamenshchik, and K. Kirsten, "One-loop effective action for Euclidean Maxwell theory on manifolds with boundary," Phys. Rev. D54 (1996) 7328-7337, hep-th/9606132.

[195] G. Esposito, A. Y. Kamenshchik, and K. Kirsten, "Casimir energy in the axial gauge," Phys. Rev. D62 (2000) 085027, hep-th/0006220

[196] G. Esposito, A. Y. Kamenshchik, I. V. Mishakov, and G. Pollifrone, "Euclidean Maxwell theory in the presence of boundaries. Part 2," Class. Quant. Grav. 11 (1994) 2939-2950, gr-qc/9506061.

[197] G. Esposito, A. Y. Kamenshchik, and G. Pollifrone, "One-loop effective action on the four-ball," Class. Quant. Grav. 13 (1996) 943-956, hep-th/9601006.

[198] G. Esposito, A. Y. Kamenshchik, and G. Pollifrone, Euclidean quantum gravity on manifolds with boundary. Kluwer, Dordrecht, 1997.

[199] G. Esposito and K. Kirsten, "Chiral bag boundary conditions on the ball," Phys. Rev. D66 (2002) 085014, hep-th/0207109.

[200] L. D. Faddeev and A. A. Slavnov, Gauge Fields. Introduction to Quantum Theory, vol. 83 of Front. Phys. Addison-Wesley, Redwood, 1990.

[201] H. Falomir, R. E. Gamboa Saravi, and E. M. Santangelo, "Dirac operator on a disk with global boundary conditions," J. Math. Phys. 39 (1998) 532-544, hep-th/9609194

[202] H. Falomir, K. Kirsten, and K. Rebora, "Divergencies in the Casimir energy for a medium with realistic ultraviolet behavior," J. Phys. A 34 (2001) 6291-6299, hep-th/0103050

[203] H. Falomir, M. A. Muschietti, and E. M. Santangelo, "Fermionic determinants for chiral bag like two-dimensional systems (an application of Seeley's developments)," J. Math. Phys. 31 (1990) 989.

[204] H. Falomir and E. M. Santangelo, "An alternative approach to chiral bag fermionic determinants," Phys. Rev. D43 (1991) 539-543.

[205] D. Fliegner, P. Haberl, M. G. Schmidt, and C. Schubert, "An improved heat kernel expansion from worldline path integrals," Discourses Math. Appl. 4 (1995) 87-99, hep-th/9411177

[206] D. Fliegner, P. Haberl, M. G. Schmidt, and C. Schubert, "The higher derivative expansion of the effective action by the string inspired method. ii," Annals Phys. 264 (1998) 51-74, hep-th/9707189

[207] V. A. Fock, "The proper time in classical and quantum mechanics," Izv. Akad. Nauk. USSR (Phys.) 4-5 (1937) 551-568.

[208] P. Forgacs, L. O'Raifeartaigh, and A. Wipf, "Scattering theory, U(1) anomaly and index theorems for compact and noncompact manifolds," Nucl. Phys. B293 (1987) 559. 
[209] E. S. Fradkin and A. A. Tseytlin, "Renormalizable asymptotically free quantum theory of gravity," Nucl. Phys B201 (1982) 469-491.

[210] E. S. Fradkin and A. A. Tseytlin, "Quantum properties of higher dimensional and dimensionally reduced supersymmetric theories," Nucl. Phys. B227 (1983) 252.

[211] E. S. Fradkin and A. A. Tseytlin, "One loop effective potential in gauged O(4) supergravity," Nucl. Phys. B234 (1984) 472.

[212] E. S. Fradkin and A. A. Tseytlin, "Nonlinear electrodynamics from quantized strings," Phys. Lett. B163 (1985) 123.

[213] E. S. Fradkin and A. A. Tseytlin, "Quantum equivalence of dual field theories," Ann. Phys. 162 (1985) 31.

[214] D. Friedan, "On two-dimensional conformal invariance and the field theory of string," Phys. Lett. B162 (1985) 102.

[215] D. Friedan, Z.-a. Qiu, and S. H. Shenker, "Conformal invariance, unitarity and two-dimensional critical exponents," Phys. Rev. Lett. 52 (1984) $1575-1578$.

[216] V. Frolov, P. Sutton, and A. Zelnikov, "The dimensional-reduction anomaly," Phys. Rev. D61 (2000) 024021, hep-th/9909086.

[217] V. P. Frolov and E. M. Serebryanyi, "Vacuum polarization in the gravitational field of a cosmic string," Phys. Rev. D35 (1987) 3779-3782.

[218] V. P. Frolov and G. A. Vilkovisky, "Quantum gravity removes classical singularities and shortens the life of black holes,". Presented at 2nd Marcel Grossmann Mtg. on Recent Developments of General Relativity, Trieste, Italy, 1979, ICTP preprint IC/79/69.

[219] V. P. Frolov and G. A. Vilkovisky, "Spherically symmetric collapse in quantum gravity," Phys. Lett. B106 (1981) 307-313.

[220] K. Fujikawa, "Path integral measure for gauge invariant fermion theories," Phys. Rev. Lett. 42 (1979) 1195.

[221] K. Fujikawa, "Path integral for gauge theories with fermions," Phys. Rev. D21 (1980) 2848.

[222] K. Fujikawa, "Comment on chiral and conformal anomalies," Phys. Rev. Lett. 44 (1980) 1733.

[223] K. Fujikawa and P. van Nieuwenhuizen, "Topological anomalies from the path integral measure in superspace," hep-th/0305144.

[224] S. A. Fulling, Aspects Of Quantum Field Theory In Curved Space-Time. Cambridge Univerisity Press, Cambridge, 1989.

[225] S. A. Fulling, "Systematics of the relationship between vacuum energy calculations and heat kernel coefficients," quant-ph/0302117. 
[226] D. V. Fursaev, "The heat kernel expansion on a cone and quantum fields near cosmic strings," Class. Quant. Grav. 11 (1994) 1431-1444, hep-th/9309050.

[227] D. V. Fursaev, "Spectral geometry and one loop divergences on manifolds with conical singularities," Phys. Lett. B334 (1994) 53-60, hep-th/9405143.

[228] D. V. Fursaev, "Spectral asymptotics of eigen-value problems with nonlinear dependence on the spectral parameter," Class. Quant. Grav. 19 (2002) 3635-3652, hep-th/0201219

[229] D. V. Fursaev, "Statistical mechanics, gravity, and Euclidean theory," Nucl. Phys. Proc. Suppl. 104 (2002) 33-62, hep-th/0107089.

[230] D. V. Fursaev and S. N. Solodukhin, "On the description of the Riemannian geometry in the presence of conical defects," Phys. Rev. D52 (1995) 2133-2143, hep-th/9501127

[231] B. Gaveau and L. S. Schulman, "Explicit time-dependent Schrödinger propagators," J. Phys. A 19 (1986) 1833.

[232] G. W. Gibbons, S. W. Hawking, and M. J. Perry, "Path integrals and the indefiniteness of the gravitational action," Nucl. Phys. B138 (1978) 141.

[233] G. W. Gibbons and M. J. Perry, "Quantizing gravitational instantons," Nucl. Phys. B146 (1978) 90.

[234] P. Gilkey, K. Kirsten, and D. Vassilevich, "Supertrace divergence terms for the Witten Laplacian," math-ph/0212023

[235] P. Gilkey, K. Kirsten, D. Vassilevich, and A. Zelnikov, "Duality symmetry of the p-form effective action and super trace of the twisted de Rham complex," Nucl. Phys. B648 (2003) 542-556, hep-th/0209125

[236] P. B. Gilkey, "Curvature and eigenvalues of the Laplacian for elliptic complexes," Adv. Math. 10 (1973) 344-382.

[237] P. B. Gilkey, "Recursion relations and the asymptotic behavior of the eigenvalues of the Laplacian," Compositio Math. 38 (1979) 201-240.

[238] P. B. Gilkey, "The spectral geometry of the higher order Laplacian," Duke Math. J. 47 (1980) 511-528.

[239] P. B. Gilkey, "Leading terms in the asymptotics of the heat equation," Contemp. Math. 73 (1988) 79-85.

[240] P. B. Gilkey, T. P. Branson, and S. Fulling, "Heat equation asymptotics of nonminimal operators on differential forms," J. Math. Phys. 32 (1991) 2089-2091.

[241] P. B. Gilkey and G. Grubb, "Logarithmic terms in asymptotic expansions of heat operator traces," Commun. Part. Diff. Eq. 23 (1998) 777-792.

[242] P. B. Gilkey and K. Kirsten, "Heat asymptotics with spectral boundary conditions. 2," math-ph/0007015. 
[243] P. B. Gilkey and L. Smith, "The eta invariant for a class of elliptic boundary value problems," Commun. Pure Appl. Math. 36 (1983) 85-131.

[244] P. Gilkey, "The spectral geometry of a Riemannian manifold," J. Diff. Geom. 10 (1975) 601-618.

[245] P. Gilkey, Invariance Theory, the Heat Equation, and the Atiya-Singer Index Theorem. CRC Press, Boca Raton, FL, 1995.

[246] P. Gilkey, K. Kirsten, and D. Vassilevich, "Divergence terms in the supertrace heat asymptotics for the de Rham complex on a manifold with boundary," math-ph/0211020

[247] P. Gilkey, K. Kirsten, and D. Vassilevich, "Heat trace asymptotics defined by transfer boundary conditions," Lett. Math. Phys. 63 (2003) 29-37, hep-th/0208130

[248] P. B. Gilkey, K. Kirsten, and D. V. Vassilevich, "Heat trace asymptotics with transmittal boundary conditions and quantum brane-world scenario," Nucl. Phys. B601 (2001) 125-148, hep-th/0101105.

[249] M. Gleiser, P. Jetzer, and M. A. Rubin, "Vacuum energy of $M^{4} \times S^{M} \times S^{N}$ in even dimensions," Phys. Rev. D36 (1987) 2429.

[250] J. Goldstone and R. L. Jaffe, "The baryon number in chiral bag models," Phys. Rev. Lett. 51 (1983) 1518.

[251] I. S. Gradshteyn and I. Ryzhik, Table of integrals, series, and products. Academic Press, San Diego, 2000.

[252] N. Graham et al., "Calculating vacuum energies in renormalizable quantum field theories: A new approach to the Casimir problem," Nucl. Phys. B645 (2002) 49-84, hep-th/0207120

[253] P. Greiner, "An asymptotic expansion for the heat kernel," Arch. Rat. Mech. Anal. 41 (1971) 163-218.

[254] P. A. Griffin and D. A. Kosower, "Curved space-time one loop gravity in a physical gauge," Phys. Lett. B233 (1989) 295.

[255] S. Groot Nibbelink, "Traces on orbifolds: Anomalies and one-loop amplitudes," hep-th/0305139.

[256] C. Grosche, "delta-prime function perturbations and neumann boundary conditions by path integration," J. Phys. A 28 (1995) L99, hep-th/9402110.

[257] G. Grubb, "Properties of normal boundary problems for elliptic even-order systems," Ann. Scuola Norm. Sup. Pisa 1 (1974) 1.

[258] G. Grubb, Functional calculus of pseudo-differential boundary problem. Birkhauser, Boston, 1996.

[259] G. Grubb, "Trace formulas for parameter-dependent pseudodifferential operators," Nucl. Phys. B (Proc. Suppl.) 104 (2002) 71-88. 
[260] G. Grubb and R. Seeley, "Asymptotic expansion of the Atiyah-Patodi-Singer operator," C. R. Acad. Sci., Paris, Ser. I 317 (1993) 1123-1126.

[261] G. Grubb and R. Seeley, "Weakly parametric pseudodifferential operators and problems," Invent. Math. 121 (1995) 481-529.

[262] G. Grubb and R. Seeley, "Zeta and eta functions for Atiyah-Patodi-Singer operators," J. Geom. Anal. 6 (1996) 31-77.

[263] G. Grubb, "Logarithmic terms in trace expansions of Atiyah-Patodi- Singer problems," math.ap/0302289, to be published in Ann. Global Anal. Geom.

[264] G. Grubb, "Spectral boundary conditions for generalizations of Laplace and Dirac operators," math.ap/0302286. to be published in Commun. Math. Phys.

[265] D. Grumiller, W. Kummer, and D. V. Vassilevich, "Dilaton gravity in two dimensions," Phys. Rept. 369 (2002) 327-430, hep-th/0204253.

[266] E. I. Guendelman, A. Leonidov, V. Nechitailo, and D. A. Owen, "On the heat kernel in covariant background gauge," Phys. Lett. B324 (1994) 160-163, hep-th/9312138

[267] Y. V. Gusev and A. I. Zelnikov, "Two-dimensional effective action for matter fields coupled to the dilaton," Phys. Rev. D61 (2000) 084010, hep-th/9910198

[268] V. P. Gusynin, "Seeley-Gilkey coefficients for the fourth order operators on a riemannian manifold," Nucl. Phys. B333 (1990) 296.

[269] V. P. Gusynin, E. V. Gorbar, and V. V. Romankov, "Heat kernel expansion for nonminimal differential operators and manifolds with torsion," Nucl. Phys. B362 (1991) 449-474.

[270] V. P. Gusynin and V. V. Kornyak, "Computation of the DeWitt-Seeley-Gilkey coefficient E(4) for nonminimal operator in curved space," Nucl. Instrum. Meth. A389 (1997) 365-369.

[271] J. Hadamard, Le Problème de Cauchi et les Èquations aux Dérivées Partielles Linéaires Hyperboliques. Hermann et Cie, Paris, 1932.

[272] T. H. Hansson and R. L. Jaffe, "The multiple reflection expansion for confined scalar, Dirac and gauge fields," Ann. Phys. 151 (1983) 204.

[273] P. Hasenfratz and J. Kuti, "The quark bag model," Phys. Rept. 40 (1978) $75-179$.

[274] S. W. Hawking, "Zeta function regularization of path integrals in curved space-time," Commun. Math. Phys. 55 (1977) 133.

[275] W. Heisenberg and H. Euler, "Folgerungen aus der Diracschen Theorie des Positrons," Z. Physik 98 (1936) 714. 
[276] S. Helgason, Groups and Geometric Analysis. Academic Press, New York, 1984.

[277] G. T. Horowitz and L. Susskind, "Bosonic M theory," J. Math. Phys. 42 (2001) 3152-3160, hep-th/0012037

[278] M. Hortacsu, "Index theorem for merons," Lett. Nuovo Cim. 36 (1983) $109-112$

[279] M. Hortacsu, K. D. Rothe, and B. Schroer, "Generalized qed in two-dimensions and functional determinants," Phys. Rev. D20 (1979) 3203.

[280] M. Hortacsu, K. D. Rothe, and B. Schroer, "Zero energy eigenstates for the Dirac boundary problem," Nucl. Phys. B171 (1980) 530.

[281] P. Hrasko and J. Balog, "The fermion boundary condition and the theta angle in qed in two-dimensions," Nucl. Phys. B245 (1984) 118.

[282] N. Hurt, Geometric quantization in action. Reidel, Dordrecht, 1983.

[283] W. Israel, "Singular hypersurfaces and thin shells in general relativity," Nuovo Cim. B44S10 (1966) 1.

[284] I. Jack and L. Parker, "Proof of summed form of proper time expansion for propagator in curved space-time," Phys. Rev. D31 (1985) 2439.

[285] M.-T. Jaekel and S. Reynaud, "Causality, stability and passivity for a mirror in vacuum," Phys. Lett. A167 (1992) 227-232, quant-ph/0101080

[286] M. Kac, "Can one hear the shape of a drum?," Amer. Math. Monthly 73 (1966) 1-23.

[287] A. Y. Kamenshchik and I. V. Mishakov, "Zeta function technique for quantum cosmology: The contributions of matter fields to the Hartle-Hawking wave function of the universe," Int. J. Mod. Phys. A7 (1992) 3713-3746.

[288] A. Y. Kamenshchik and I. V. Mishakov, "Fermions in one loop quantum cosmology," Phys. Rev. D47 (1993) 1380-1390.

[289] A. Y. Kamenshchik and I. V. Mishakov, "Fermions in one loop quantum cosmology. 2: The problem of correspondence between covariant and noncovariant formalisms," Phys. Rev. D49 (1994) 816-824.

[290] R. Kantowski and K. A. Milton, "Casimir energies in $M^{4} \times S^{N}$ for even N. Green's function and zeta function techniques," Phys. Rev. D36 (1987) $3712-3721$.

[291] G. Kennedy, "Boundary terms in the Schwinger-DeWitt expansion: flat space results," J. Phys. A 11 (1978) L173-L178.

[292] G. Kennedy, R. Critchley, and J. S. Dowker, "Finite temperature field theory with boundaries: Stress tensor and surface action renormalization," Ann.

Phys. 125 (1980) 346. 
[293] N. R. Khusnutdinov, "Semiclassical wormholes," hep-th/0304176.

[294] N. R. Khusnutdinov and S. V. Sushkov, "Ground state energy in a wormhole space-time," Phys. Rev. D65 (2002) 084028, hep-th/0202068.

[295] K. Kikkawa, T. Kubota, S. Sawada, and M. Yamasaki, "Spontaneous compactification in generalized Candelas- Weinberg models," Nucl. Phys. B260 (1985) 429.

[296] K. Kirsten, "The a(5) heat kernel coefficient on a manifold with boundary," Class. Quant. Grav. 15 (1998) L5, hep-th/9708081

[297] K. Kirsten, Spectral functions in mathematics and physics. Chapman \& Hall/CRC, Boca Raton, FL, 2001.

[298] K. Kirsten and G. Cognola, "Heat kernel coefficients and functional determinants for higher spin fields on the ball," Class. Quant. Grav. 13 (1996) 633-644, hep-th/9508088.

[299] K. Kirsten and A. McKane, "Functional determinants by contour integration methods," math-ph/0305010.

[300] I. R. Klebanov and A. A. Tseytlin, "D-branes and dual gauge theories in type 0 strings," Nucl. Phys. B546 (1999) 155-181, hep-th/9811035.

[301] S. Kobayashi and K. Nomizu, Foundations of Differential Geometry, Vols. I and II. Interscience, New York, 1969.

[302] Y. I. Kogan and N. A. Voronov, "Spontaneous compactification in the Kaluza-Klein models and the Casimir effect," JETP Lett. 38 (1983) 311.

[303] W. Kummer, H. Liebl, and D. V. Vassilevich, "Hawking radiation for non-minimally coupled matter from generalized 2d black hole models," Mod. Phys. Lett. A12 (1997) 2683-2690, hep-th/9707041.

[304] W. Kummer and D. V. Vassilevich, "Effective action and Hawking radiation for dilaton coupled scalars in two dimensions," Phys. Rev. D60 (1999) 084021, hep-th/9811092.

[305] W. Kummer and D. V. Vassilevich, "Hawking radiation from dilaton gravity in (1+1) dimensions: A pedagogical review," Annalen Phys. 8 (1999) 801-827, gr-qc/9907041.

[306] W. Kummer and D. V. Vassilevich, "Renormalizability of the open string sigma model and emergence of D-branes," J. High Energy Phys. 07 (2000) 012, hep-th/0006108.

[307] J. Louko, "Quantum cosmology with electromagnetism," Phys. Rev. D38 (1988) 478.

[308] H. Luckock, "Mixed boundary conditions in quantum field theory," J. Math. Phys. 32 (1991) 1755. 
[309] M. Lüscher, K. Symanzik, and P. Weisz, "Anomalies of the free loop wave equation in the WKB approximation," Nucl. Phys. B173 (1980) 365.

[310] V. D. Lyakhovsky, N. N. Shtykov, and D. V. Vassilevich, "DeWitt-Schwinger expansion for projective and Grassmann spaces," Lett. Math. Phys. 21 (1991) 89-95.

[311] V. D. Lyakhovsky and D. V. Vassilevich, "Algebraic approach to Kaluza-Klein models," Lett. Math. Phys. 17 (1989) 109-115.

[312] Z.-Q. Ma, "Axial anomaly and index theorem for a two-dimensional disk with boundary," J. Phys. A19 (1986) L317.

[313] V. N. Marachevsky and D. Vassilevich, "Diffeomorphism invariant eigenvalue problem for metric perturbations in a bounded region," Class. Quant. Grav. 13 (1996) 645-652, gr-qc/9509051.

[314] P. O. Mazur and E. Mottola, "The gravitational measure, solution of the conformal factor problem and stability of the ground state of quantum gravity," Nucl. Phys. B341 (1990) 187-212.

[315] D. M. McAvity and H. Osborn, "A DeWitt expansion of the heat kernel for manifolds with a boundary," Class. Quant. Grav. 8 (1991) 603-638.

[316] D. M. McAvity and H. Osborn, "Asymptotic expansion of the heat kernel for generalized boundary conditions," Class. Quant. Grav. 8 (1991) 1445-1454.

[317] D. M. McAvity and H. Osborn, "Quantum field theories on manifolds with curved boundaries: Scalar fields," Nucl. Phys. B394 (1993) 728-790, cond-mat/9206009.

[318] D. M. McAvity, "Heat kernel asymptotics for mixed boundary conditions," Class. Quant. Grav. 9 (1992) 1983-1998.

[319] H. P. McKean and I. M. Singer, "Curvature and the eigenvalues of the Laplacian," J. Diff. Geom. 1 (1967) 43-69.

[320] J. Melmed, "Conformal invariance and the regularized one loop effective action," J. Phys. A21 (1988) L1131-L1134.

[321] A. Mikovic and V. Radovanovic, "One-loop effective action for spherical scalar field collapse," Class. Quant. Grav. 15 (1998) 827-838, hep-th/9706066

[322] K. A. Milton, The Casimir effect: Physical manifestations of zero-point energy. World Scientific, River Edge, 2001.

[323] K. A. Milton, "Semiclassical electron models: Casimir selfstress in dielectric and conducting balls," Ann. Phys. 127 (1980) 49.

[324] K. A. Milton, "Calculating casimir energies in renormalizable quantum field theory," hep-th/0210081. 
[325] S. Minakshisundaram, "A generalization of Epstein zeta functions," Can. J. Math. 1 (1949) 320-327.

[326] S. Minakshisundaram and A. Pleijel, "Some properties of the eigenfunctions of the Laplace operator on Riemannian manifolds," Can. J. Math. 1 (1949) $242-256$.

[327] A. V. Mishchenko and Y. A. Sitenko, "Spectral boundary conditions and index theorem for two- dimensional compact manifold with boundary," Ann. Phys. 218 (1992) 199-232.

[328] I. G. Moss, "Boundary terms in the heat kernel expansion," Class. Quant. Grav. 6 (1989) 759.

[329] I. G. Moss and J. S. Dowker, "The correct B(4) coefficient," Phys. Lett. B229 (1989) 261.

[330] I. G. Moss, "Heat kernel expansions for distributional backgrounds," Phys. Lett. B491 (2000) 203-206, hep-th/0007185.

[331] I. G. Moss and W. Naylor, "Diagrams for heat kernel expansions," Class. Quant. Grav. 16 (1999) 2611-2624, gr-qc/0101125.

[332] I. G. Moss, W. Naylor, W. Santiago-German, and M. Sasaki, "Bulk quantum effects for de Sitter branes in $\operatorname{AdS}(5), "$ hep-th/0302143.

[333] I. G. Moss and S. J. Poletti, "Conformal anomalies on Einstein spaces with boundary," Phys. Lett. B333 (1994) 326-330, gr-qc/9405044.

[334] I. G. Moss and P. J. Silva, "BRST invariant boundary conditions for gauge theories," Phys. Rev. D55 (1997) 1072-1078, gr-qc/9610023

[335] E. Mottola, "Functional integration over geometries," J. Math. Phys. 36 (1995) 2470-2511, hep-th/9502109

[336] V. Mukhanov, A. Wipf, and A. Zelnikov, "On 4-d hawking radiation from effective action," Phys. Lett. B332 (1994) 283-291, hep-th/9403018.

[337] R. I. Nepomechie, "Calculating heat kernels," Phys. Rev. D31 (1985) 3291.

[338] V. V. Nesterenko, G. Lambiase, and G. Scarpetta, "Casimir energy of a semi-circular infinite cylinder," J. Math. Phys. 42 (2001) 1974-1986, hep-th/0005257

[339] V. V. Nesterenko and I. G. Pirozhenko, "Spectral zeta functions for a cylinder and a circle," J. Math. Phys. 41 (2000) 4521-4531, hep-th/9910097.

[340] V. V. Nesterenko, I. G. Pirozhenko, and J. Dittrich, "Nonsmoothness of the boundary and the relevant heat kernel coefficients," Class. Quant. Grav. 20 (2003) 431-456, hep-th/0207038.

[341] A. J. Niemi and G. W. Semenoff, "Index theorems on open infinite manifolds," Nucl. Phys. B269 (1986) 131. 
[342] M. Ninomiya and C.-I. Tan, "Axial anomaly and index theorem for manifolds with boundary," Nucl. Phys. B257 (1985) 199.

[343] S. Nojiri, S. D. Odintsov, and S. Zerbini, "Bulk versus boundary (gravitational Casimir) effects in quantum creation of inflationary brane world universe," Class. Quant. Grav. 17 (2000) 4855-4866, hep-th/0006115

[344] S. Nojiri and S. D. Odintsov, "Trace anomaly and non-local effective action for 2d conformally invariant scalar interacting with dilaton," Mod. Phys. Lett. A12 (1997) 2083-2088, hep-th/9706009.

[345] S. Nojiri and S. D. Odintsov, "Trace anomaly induced effective action for $2 \mathrm{~d}$ and 4d dilaton coupled scalars," Phys. Rev. D57 (1998) 2363-2371, hep-th/9706143

[346] Y. Novozhilov, A. Pronko, and D. Vassilevich, "Extended chiral transformations including diquark fields as parameters," Phys. Lett. B343 (1995) 358-362, hep-ph/9411421.

[347] Y. V. Novozhilov and D. V. Vassilevich, "Induced classical gravity," Lett. Math. Phys. 21 (1991) 253-271.

[348] Y. V. Novozhilov and D. V. Vassilevich, "Remarks on the conformal factor problem in gravity," Int. J. Mod. Phys. A6 (1991) 3347-3353.

[349] Y. N. Obukhov, "The geometrical approach to antisymmetric tensor field theory," Phys. Lett. B109 (1982) 195-199.

[350] Y. N. Obukhov, "Spectral geometry of the Riemann-Cartan space-time," Nucl. Phys. B212 (1983) 237-254.

[351] S. D. Odintsov, "Renormalization group and boundary terms," Class. Quant. Grav. 7 (1990) 445.

[352] H. Osborn, "Weyl consistency conditions and a local renormalization group equation for general renormalizable field theories," Nucl. Phys. B363 (1991) $486-526$.

[353] A. A. Osipov and B. Hiller, "Inverse mass expansion of the one-loop effective action," Phys. Lett. B515 (2001) 458-462.

[354] A. A. Osipov and B. Hiller, "Large mass invariant asymptotics of the effective action," Phys. Rev. D64 (2001) 087701, hep-th/0106226

[355] A. A. Osipov and B. Hiller, "Generalized proper-time approach for the case of broken isospin symmetry," Phys. Rev. D63 (2001) 094009, hep-ph/0012294

[356] H. Pagels and E. Tomboulis, "Vacuum of the quantum Yang-Mills theory and magnetostatics," Nucl. Phys. B143 (1978) 485.

[357] L. Parker and D. J. Toms, "Renormalization group analysis of grand unified theories in curved space-time," Phys. Rev. D29 (1984) 1584. 
[358] L. Parker and D. J. Toms, "Explicit curvature dependence of coupling constants," Phys. Rev. D31 (1985) 2424.

[359] L. Parker and D. J. Toms, "New form for the coincidence limit of the Feynman propagator, or heat kernel, in curved space-time," Phys. Rev. D31 (1985) 953.

[360] A. M. Polyakov, "Quantum geometry of bosonic strings," Phys. Lett. B103 (1981) 207-210.

[361] P. I. Pronin and K. V. Stepanyants, "One-loop divergences in theories with an arbitrary nonminimal operator in curved space," Theor. Math. Phys. 110 (1997) 277-294.

[362] L. Randall and R. Sundrum, "An alternative to compactification," Phys. Rev. Lett. 83 (1999) 4690-4693, hep-th/9906064.

[363] L. Randall and R. Sundrum, "A large mass hierarchy from a small extra dimension," Phys. Rev. Lett. 83 (1999) 3370-3373, hep-ph/9905221.

[364] M. Reed and B. Simon, Methods of Modern Mathematical Physics, vol. II: Fourier Analysis and Self-Adjointness. Academic Press, New York, 1975.

[365] M. Rho, A. S. Goldhaber, and G. E. Brown, "Topological soliton bag model for baryons," Phys. Rev. Lett. 51 (1983) 747-750.

[366] R. J. Riegert, "A nonlocal action for the trace anomaly," Phys. Lett. B134 (1984) 56-60.

[367] G. Roepstorff, Path integral approach to quantum physics: An Introduction. Springer, Berlin, 1994.

[368] V. N. Romanov and A. S. Schwarz, "Anomalies and elliptic operators," Teor. Mat. Fiz. 41 (1979) 190.

[369] H. Römer and B. Schroer, "'Fractional winding numbers' and surface effects," Phys. Lett. B71 (1977) 182.

[370] V. A. Rubakov, "Large and infinite extra dimensions: An introduction," Phys. Usp. 44 (2001) 871-893, hep-ph/0104152.

[371] V. A. Rubakov and M. E. Shaposhnikov, "Do we live inside a domain wall?," Phys. Lett. B125 (1983) 136-138.

[372] M. A. Rubin and B. D. Roth, "Fermions and stability in five-dimensional Kaluza-Klein theory," Phys. Lett. B127 (1983) 55.

[373] A. Salam and J. Strathdee, "On Kaluza-Klein theory," Annals Phys. 141 (1982) 316-352.

[374] M. Scandurra, "The ground state energy of a massive scalar field in the background of a semi-transparent spherical shell," J. Phys. A32 (1999) 5679-5691, hep-th/9811164 
[375] K. Schleich, "Conformal rotation in perturbative gravity," Phys. Rev. D36 (1987) 2342-2363.

[376] K. Schleich and D. M. Witt, "Generalized sums over histories for quantum gravity. 1. smooth conifolds," Nucl. Phys. B402 (1993) 411-468, gr-qc/9307015

[377] A. S. Schwarz, "The partition function of a degenerate functional," Commun. Math. Phys. 67 (1979) 1-16.

[378] A. S. Schwarz and A. A. Tseytlin, "Dilaton shift under duality and torsion of elliptic complex," Nucl. Phys. B399 (1993) 691-708, hep-th/9210015.

[379] J. Schwinger, "On gauge invariance and vacuum polarization," Phys. Rev. 82 (1951) 664-679.

[380] J. S. Schwinger, L. L. DeRaad, and K. A. Milton, "Casimir effect in dielectrics," Annals Phys. 115 (1979) 1-23.

[381] R. Seeley, "Singular integrals and boundary value problems," Amer. J. Math. 88 (1966) 781-809.

[382] R. Seeley, "The resolvent of an elliptic boundary value problem," Amer. J. Math. 91 (1969) 889-920.

[383] R. T. Seeley, "Complex powers of an elliptic operator," in Singular Integrals, Chicago 1966, vol. 10 of Proc. Symp. Pure Math., pp. 308-315. Amer. Math. Soc., Providence, 1968.

[384] R. T. Seeley, "Trace expansion for mixed boundary problems," Nucl. Phys. B (Proc. Suppl.) 104 (2002) 201-203.

[385] N. Shtykov and D. V. Vassilevich, "The heat kernel for deformed spheres," J. Phys. A28 (1995) L37-L44, hep-th/9411214.

[386] W. Siegel, "Hidden ghosts," Phys. Lett. B93 (1980) 170.

[387] R. Slansky, "Group theory for unified model building," Phys. Rept. 79 (1981) 1-128.

[388] L. Smith, "The asymptotics of the heat equation for a boundary value problem," Invent. Math. 63 (1981) 467-493.

[389] D. D. Sokolov and A. A. Starobinsky, "Structure of the curvature tensor on conical singularities," Dokl. Akad. Nauk. USSR 234 (1977) 1043.

[390] S. N. Solodukhin, "Exact solution for a quantum field with delta-like interaction," Nucl. Phys. B541 (1999) 461-482, hep-th/9801054.

[391] A. Sommerfeld, "Zur analytischen Theorie der Wärmeleitung," Math. Ann. 45 (1894) 263-257.

[392] K. Stewartson and R. Waechter, "On hearing the shape of a drum: further results," Math. Proc. Camb. Philos. Soc. 69 (1971) 353-363. 
[393] S. V. Sushkov, "New form of the renormalization counterterms for a scalar field," Int. J. Mod. Phys. A17 (2002) 820-824.

[394] K. Symanzik, "Schrödinger representation and Casimir effect in renormalizable quantum field theory," Nucl. Phys. B190 (1981) 1.

[395] J. L. Synge, "A characteristic function in Riemannian space and its application to the solution of geodesic triangles," Proc. London. Math. Soc. 32 (1931) 241-258.

[396] T. R. Taylor and G. Veneziano, "Quantum gravity at large distances and the cosmological constant," Nucl. Phys. B345 (1990) 210-230.

[397] S. Theberge, A. W. Thomas, and G. A. Miller, "The cloudy bag model. 1. the $(3,3)$ resonance," Phys. Rev. D22 (1980) 2838.

[398] H.-S. Tsao, "Conformal anomalies in a general background metric," Phys. Lett. B68 (1977) 79.

[399] A. E. M. van de Ven, "Index-free heat kernel coefficients," Class. Quant. Grav. 15 (1998) 2311, hep-th/9708152.

[400] D. V. Vassilevich, "Topologically nontrivial supermembranes," Class. Quant. Grav. 8 (1991) 2163-2168.

[401] D. V. Vassilevich, "Abelian gauge theories on homogeneous spaces," Lett. Math. Phys. 26 (1992) 147-152.

[402] D. V. Vassilevich, "One loop quantum gravity on de Sitter space," Int. J. Mod. Phys. A8 (1993) 1637-1652.

[403] D. V. Vassilevich, "Quantum gravity on $C P^{2}$," Int. J. Mod. Phys. D2 (1993) $135-147$.

[404] D. V. Vassilevich, "Vector fields on a disk with mixed boundary conditions," J. Math. Phys. 36 (1995) 3174-3182, gr-qc/9404052.

[405] D. V. Vassilevich, "The Faddeev-Popov trick in the presence of boundaries," Phys. Lett. B421 (1998) 93-98, hep-th/9709182.

[406] D. V. Vassilevich, "Spectral branes," J. High Energy Phys. 03 (2001) 023, hep-th/0102091

[407] D. V. Vassilevich, V. D. Lyakhovsky, and N. N. Shtykov, "Rescaling of the metric, invariant operators, and spontaneous compactification," Theor. Math. Phys. 77 (1988) 1064-1070.

[408] D. V. Vassilevich and N. N. Shtykov, "Conformal anomaly in the Kaluza-Klein models with nonsymmetric homogeneous spaces," Sov. J. Nucl. Phys. 53 (1991) 542-545.

[409] D. V. Vassilevich and A. Zelnikov, "Discrete symmetries of functional determinants," Nucl. Phys. B594 (2001) 501-517, hep-th/0009084. 
[410] D. V. Vassilevich, "Heat kernel for antisymmetric tensor field on a disk," Phys. Lett. B348 (1995) 39-43, gr-qc/9411062.

[411] D. V. Vassilevich, "On gauge-independence in quantum gravity," Nucl. Phys. B454 (1995) 685-700, hep-th/9509069.

[412] D. V. Vassilevich, "On gauge invariant boundary conditions for 2-d gravity with dynamical torsion," Mod. Phys. Lett. A10 (1995) 2239-2244, hep-th/9504011

[413] D. V. Vassilevich, "QED on curved background and on manifolds with boundaries: Unitarity versus covariance," Phys. Rev. D52 (1995) 999-1010, gr-qc/9411036

[414] D. V. Vassilevich, "Spectral geometry for strings and branes," Nucl. Phys. Proc. Suppl. 104 (2002) 208-211, hep-th/0107083.

[415] M. S. Volkov and A. Wipf, "Black hole pair creation in de Sitter space: A complete one-loop analysis," Nucl. Phys. B582 (2000) 313-362, hep-th/0003081

[416] R. Waechter, "On hearing the shape of a drum: an extension to higher dimensions," Math. Proc. Camb. Philos. Soc. 72 (1972) 439-447.

[417] J. Wess and B. Zumino, "Consequences of anomalous Ward identities," Phys. Lett. B37 (1971) 95.

[418] H. Weyl, "Das asymptotische Verteilungsgesetz der Eigenwerte linearer partieler Differentialgleichungen," Math. Ann. 71 (1912) 441-479.

[419] H. Weyl, "Das asymptotische Verteilungsgesetz der Eigenschwingungen eines beliebig gestalteten elastischen Körpers," Rend. Circ. Mat. Palermo 39 (1915) 1-50.

[420] C. Wiesendanger and A. Wipf, "Running coupling constants from finite size effects," Ann. Phys. 233 (1994) 125-161.

[421] A. Wipf and S. Durr, "Gauge theories in a bag," Nucl. Phys. B443 (1995) 201-232, hep-th/9412018

[422] E. Witten, "Constraints on supersymmetry breaking," Nucl. Phys. B202 (1982) 253.

[423] E. Witten, "Supersymmetry and Morse theory," J. Diff. Geom. 17 (1982) 661-692.

[424] E. Witten, "Global aspects of current algebra," Nucl. Phys. B223 (1983) $422-432$.

[425] S. Yajima, "Evaluation of heat kernel in curved space," Class. Quant. Grav. 5 (1988) L207.

[426] S. Yajima, "Mixed anomalies in four-dimensional and six-dimensional space with torsion," Prog. Theor. Phys. 79 (1988) 535. 
[427] S. Yajima, "Evaluation of Heat kernel in Riemann-Cartan space," Class. Quant. Grav. 13 (1996) 2423-2436.

[428] J. Zinn-Justin, "Chiral anomalies and topology," hep-th/0201220. Contributed to Autumn School 2001: Topology and Geometry in Physics, Rot an der Rot, Germany. 